\title{
Assessment of Charging Infrastructure for Plug-in Electric Vehicles at Naval Air Station Whidbey Island: Task 3
}

Stephen Schey

Jim Francfort

July 2015

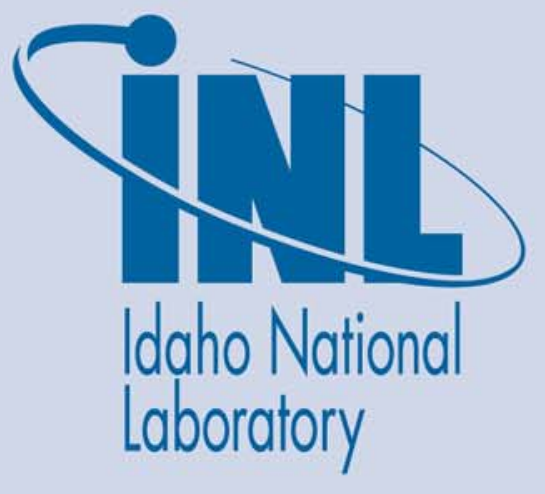

The INL is a U.S. Department of Energy National Laboratory operated by Battelle Energy Alliance 


\section{DISCLAIMER}

This information was prepared as an account of work sponsored by an agency of the U.S. Government. Neither the U.S. Government nor any agency thereof, nor any of their employees, makes any warranty, expressed or implied, or assumes any legal liability or responsibility for the accuracy, completeness, or usefulness, of any information, apparatus, product, or process disclosed, or represents that its use would not infringe privately owned rights. References herein to any specific commercial product, process, or service by trade name, trade mark, manufacturer, or otherwise, does not necessarily constitute or imply its endorsement, recommendation, or favoring by the U.S. Government or any agency thereof. The views and opinions of authors expressed herein do not necessarily state or reflect those of the U.S. Government or any agency thereof. 


\title{
Assessment of Charging Infrastructure for Plug-in Electric Vehicles at Naval Air Station Whidbey Island: Task 3
}

\author{
Stephen Schey \\ Jim Francfort ${ }^{2}$ \\ ${ }^{1}$ Stephen Schey, Project Manager, Infrastructure Planning and Analysis; Intertek Testing Services, \\ North America; Phoenix, AZ \\ ${ }^{2}$ Jim Francfort, Vehicle Systems Principal Investigator; Idaho National Laboratory operated by \\ Battelle energy Alliance; Idaho Falls, ID
}

July 2015

Idaho National Laboratory
Idaho Falls, Idaho 83415

http://avt.inl.gov

Prepared for the

U.S. Department of Energy

Office of Nuclear Energy

Under DOE Idaho Operations Office

Contract DE-AC07-05ID14517 


\section{ABSTRACT}

Battelle Energy Alliance, LLC, managing and operating contractor for the U.S. Department of Energy's Idaho National Laboratory, is the lead laboratory for U.S. Department of Energy Advanced Vehicle Testing. Battelle Energy Alliance, LLC contracted with Intertek Testing Services, North America (Intertek) to conduct several U.S. Department of Defense base studies to identify potential U.S. Department of Defense transportation systems that are strong candidates for introduction or expansion of plug-in electric vehicles (PEVs).

Task 1 consisted of a survey of the non-tactical fleet of vehicles at NASWI to begin the review of vehicle mission assignments and types of vehicles in service. Task 2 selected vehicles for further monitoring and involved identifying daily operational characteristics of these select vehicles. Data logging of vehicle movements was initiated in order to characterize the vehicle's mission.

The Task 3 Vehicle Utilization report provided the results of the data analysis and observations related to the replacement of current vehicles with PEVs. This report provides an assessment of charging infrastructure required to support the suggested PEV replacements.

Intertek acknowledges the support of Idaho National Laboratory, Naval Facilities Engineering Command (NAVFAC) Northwest, and Naval Air Station Whidbey Island fleet managers and personnel for participation in this study.

Intertek is pleased to provide this report and is encouraged by enthusiasm and support from NAVFAC personnel. 


\section{EXECUTIVE SUMMARY}

Federal agencies are mandated ${ }^{a}$ to purchase alternative-fuel vehicles, increase use of alternative fuels, and reduce consumption of petroleum used for transportation. Available plug-in electric vehicles (PEVs) provide an attractive option in the selection of alternative fuel vehicles. PEVs, which consist of both battery electric vehicles (BEVs) and plug-in hybrid electric vehicles (PHEVs), have significant advantages over internal combustion vehicles in terms of energy efficiency, reduced petroleum consumption, reduced production of greenhouse gas (GHG) emissions, and they provide performance benefits with quieter, smoother operation. This study is intended to evaluate the extent to which Naval Air Station Whidbey Island (NASWI) could convert part or all of their fleet of vehicles from petroleum-fueled vehicles to PEVs.

BEVs provide the greatest benefit when it comes to fuel and emissions savings because all the motive power is provided by the energy stored in the onboard battery pack. These vehicles use no petroleum and emit no pollutants at their point of use. PHEVs provide similar savings when their battery provides the motive power but they also have the ability to extend their operating range with an onboard internal combustion engine. Because a PHEV can meet all transportation range needs, the adoption of a PHEV will be dependent upon its ability to meet other transportation needs such as cargo or passenger carrying. Operation of PHEVs in battery-only mode can be increased with opportunity charging at available charging stations. However, it should be noted that not all PHEVs have a mode in which the battery provides all of the motive power at all speeds. The Task 3 report on Vehicle Utilization focused on the mission requirements of the fleet of vehicles. The objective is to identify vehicles that may be replaced with PEVs with emphasis on BEVs that provide maximum benefit. This report follows the vehicle use analysis with an evaluation of the electric vehicle charging infrastructure required to support those PEVs.

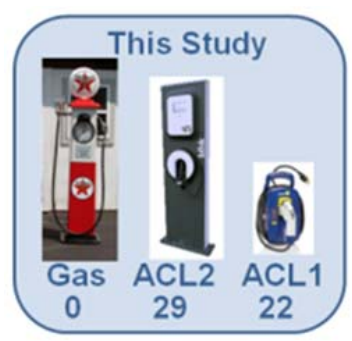

The Task 3 report on Vehicle Utilization observes that a mix of BEVs and PHEVs are capable of performing most of the required missions. Replacement of vehicles in the current fleet could result in significant reductions in the emission of GHGs and in petroleum use, as well as reduced fleet operating costs. PEVs currently commercially available cannot replace certain vehicles and missions, such as those requiring heavy-duty trucks and specialty usage vehicles. However, based upon the data collected for the monitored vehicles, all 60 vehicles have potential replacement PEVs available and the 60 -vehicle fleet subset could possibly consist of 32 BEVs, and 28 PHEVs. This report shows that 29 AC Level 2 and 22 AC Level 1 charging stations should be sufficient to support these PEVs.

\footnotetext{
${ }^{a}$ Energy Policy act of 1992, Energy Policy Act of 2005, Executive Order 13423, Energy Independence and Security Act of 2007.
} 
The monitored vehicles represent 60 vehicles of 175 on-road rated vehicles in these represented fleets. Assuming that the balance of these fleets operates in a manner similar to those monitored and without consideration of specific cargo or other mission requirements not previously identified, Intertek suggests the total fleet composition could consist of 92 BEVs and 83 PHEVs. This report suggests that 70 AC Level 2 and 58 AC Level 1 charging stations

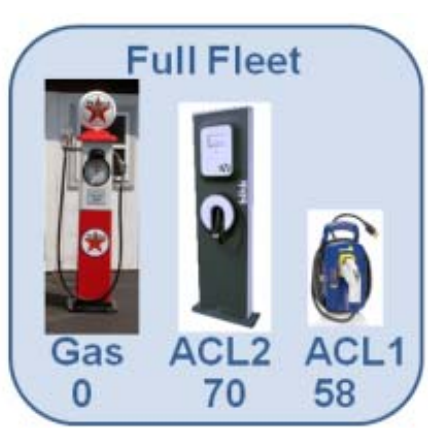
should be sufficient to support these PEVs.

Installation of PEV charging stations require pre-planning and this report provides some highlighted area of interest in this preparation. In general, electrical supply is not a concern but location relative to that supply is a significant cost factor for installation. Several potential sites are identified for charging station locations.

The information presented in this report and the prior Task 3 report on Vehicle Utilization will be considered in the Task 4 effort to identify an adoption approach for incorporating PEVs into the fleet. 


\section{CONTENTS}

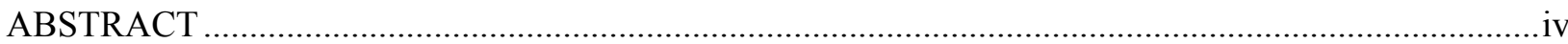

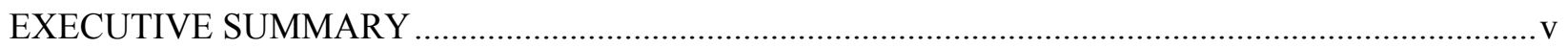

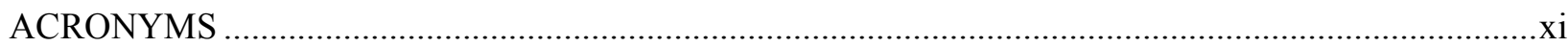

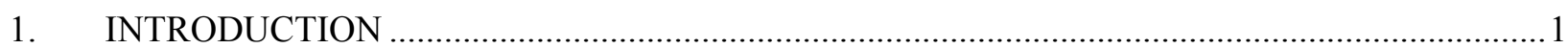

2. FLEET VEHICLE PLUG-IN ELECTRIC VEHICLE RECOMMENDATIONS ...........................2

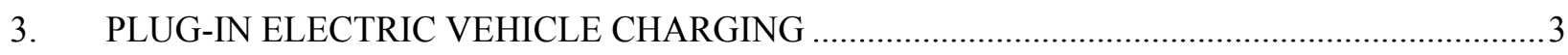

$3.1 \quad$ Electric Vehicle Supply Equipment Design ......................................................................

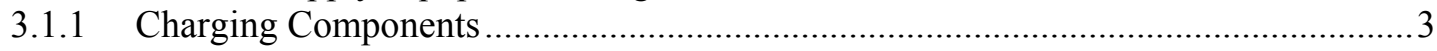

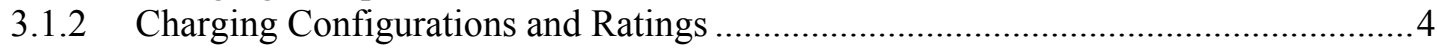

3.2 Electric Vehicle Supply Equipment Stations...........................................................

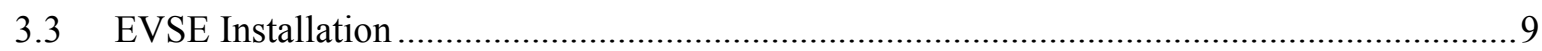

3.4 EVSE Advanced Design Considerations....................................................................... 12

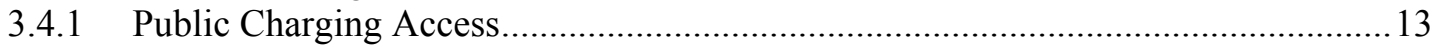

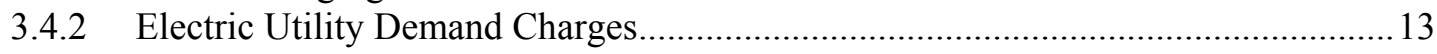

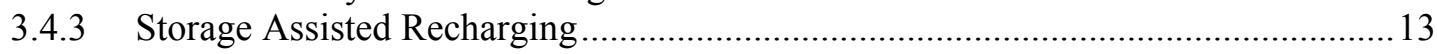

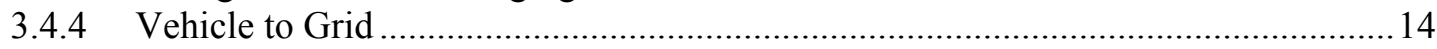

4. FLEET VEHICLE CHARGING ANALYSIS .......................................................................... 16

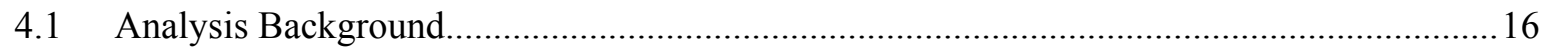

4.2 Plug-in Electric Vehicle Charging Analysis Results - Commands.................................... 18

4.2.1 Commands Group Recommended Plug-in Electric Vehicles for Monitored

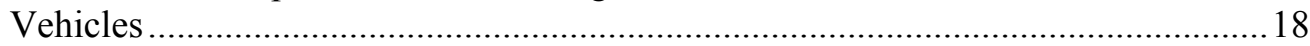

4.2.2 Commands Group Available Charge Time ......................................................... 19

4.2.3 Commands Group Electric Vehicle Supply Equipment Type Information................19

4.2.4 Commands Group Electric Vehicle Supply Equipment charging locations...............20

4.2.5 Commands Group Electric Vehicle Supply Equipment Observations ......................22

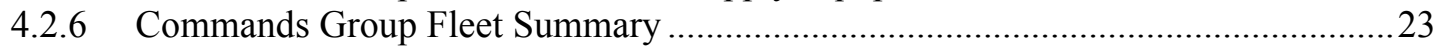

4.3 Analysis Results - Departments Group.........................................................................23

4.3.1 Departments Group recommended Plug-in Electric Vehicles for monitored

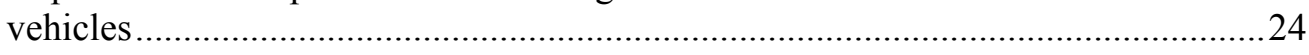

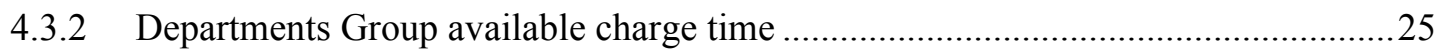

4.3.3 Departments Group Electric Vehicle Supply Equipment Type

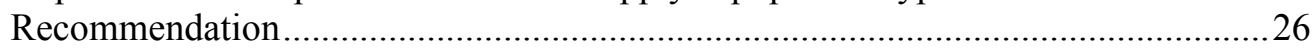

4.3.4 Departments Group Electric Vehicle Supply Equipment Charging Locations ...........26

4.3.5 Departments Group Electric Vehicle Supply Equipment observation .....................29

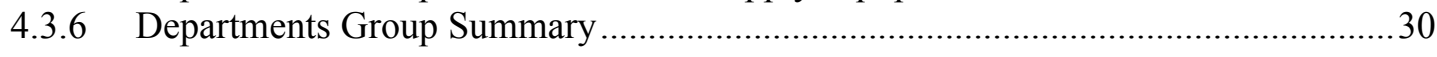

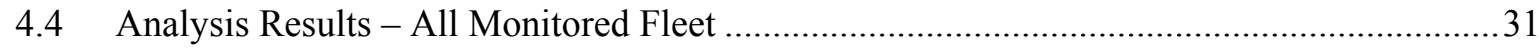

4.4.1 Aggregated Fleet available charge time ............................................................... 31

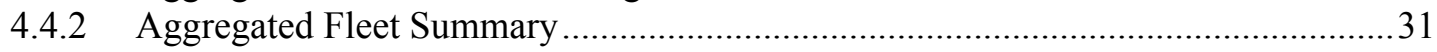

4.5 Public Charging Electric Vehicle Supply Equipment Locations......................................... 32 
5. NAVAL AIR STATION WHIDBEY ISLAND ELECTRIC VEHICLE SUPPLY

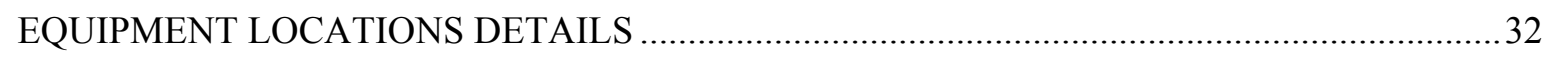

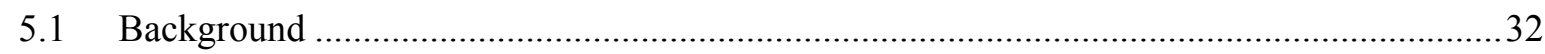

5.2 Naval Air Station Whidbey Island Electric Vehicle Supply Equipment Locations ...............32

5.2.1 Commands and EVSE Locations ............................................................................ 32

5.2.2 Departments Group Home Base Electric Vehicle Supply Equipment

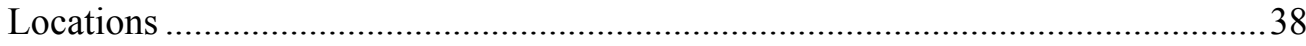

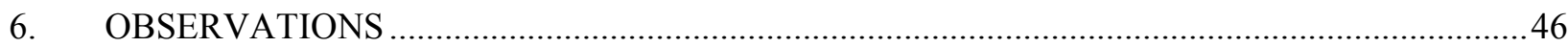

\section{FIGURES}

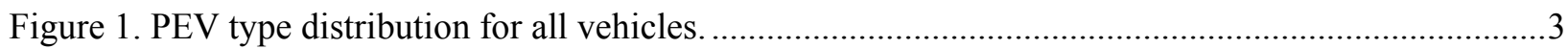

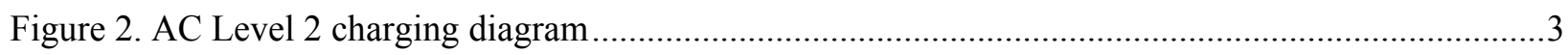

Figure 3. Society of Automotive Engineers charging configurations and ratings terminology ...................4

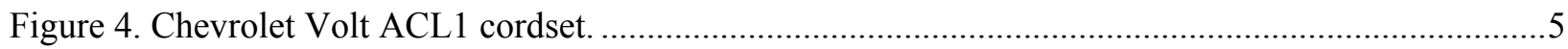

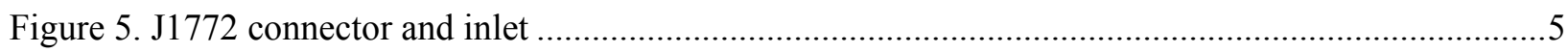

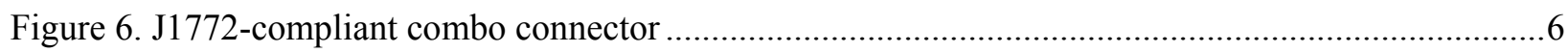

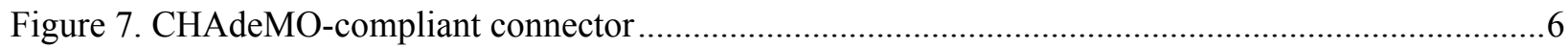

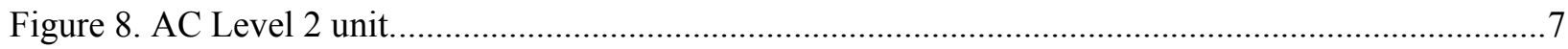

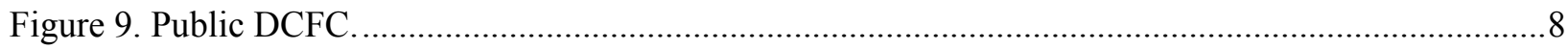

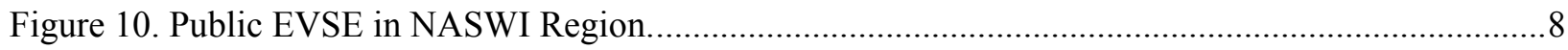

Figure 11. Installation process for commercial fleet operation.......................................................... 10

Figure 12. Typical fleet charging installation................................................................................... 12

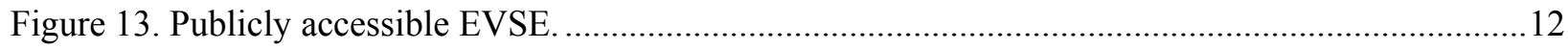

Figure 14. Conceptual design storage assisted recharging................................................................ 14

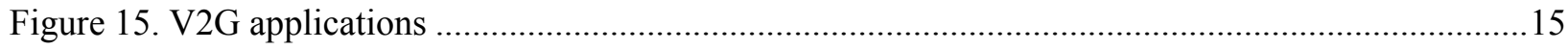

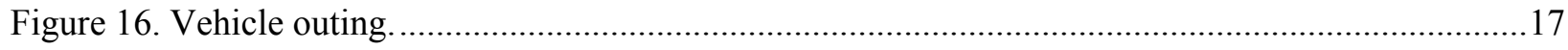

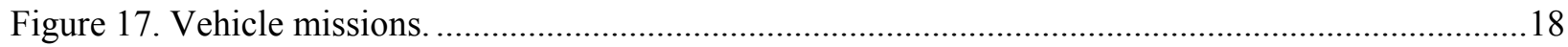

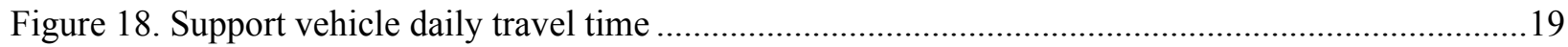

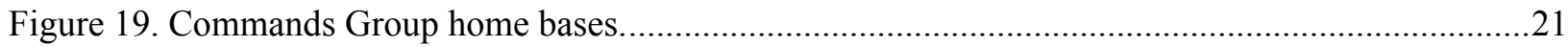

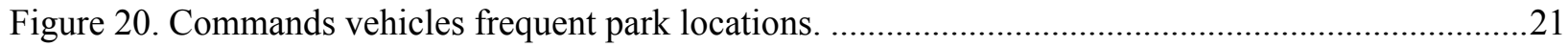

Figure 21. Commands Group vehicle stop locations by duration. .......................................................22

Figure 22. Pool, support, and enforcement vehicle daily travel minutes (all vehicles)...........................26

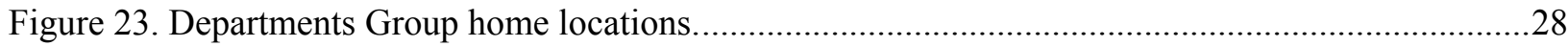

Figure 24. Departments Group vehicle stop locations by duration. .....................................................28

Figure 25. Departments Group vehicle stop locations on base greater than two hours.............................29 
Figure 26. EVSE potential location near Bldg 385 for numerous vehicles............................................33

Figure 27. EVSE potential location near Bldg 410 for numerous vehicles.............................................33

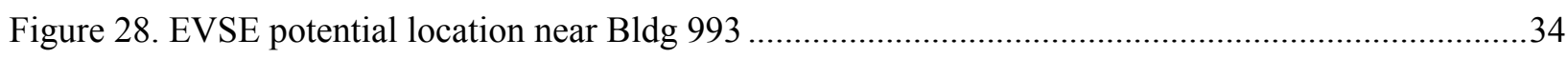

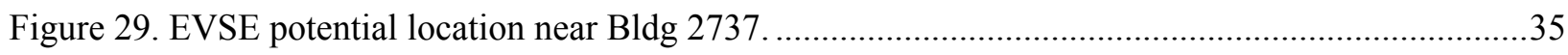

Figure 30. EVSE potential location near Bldg 386 for Vehicle G10-7547F...........................................35

Figure 31. EVSE potential location near Bldg 975 for Vehicle G41-2911M. ..........................................36

Figure 32. EVSE potential location near Bldg 124 for Vehicle G41-3159P..............................................36

Figure 33. EVSE potential location near Bldg 2544 for Vehicle G41-1351G...........................................37

Figure 34. EVSE potential location near Bldg 2547 for Vehicle G41-1136K..........................................37

Figure 35. EVSE potential location near Bldg 2593 for Vehicle G10-1140M. .......................................37

Figure 36. EVSE potential location near Bldg 2642 for Vehicle G43-3437B........................................38

Figure 37. EVSE potential location near Bldg 2897 for Vehicle G61-0513K.........................................38

Figure 38. EVSE potential location near Bldg 124 for numerous vehicles..............................................39

Figure 39. EVSE potential location near Bldg 103 for numerous vehicles..............................................40

Figure 40. EVSE potential location near Bldg 994 for numerous vehicles............................................40

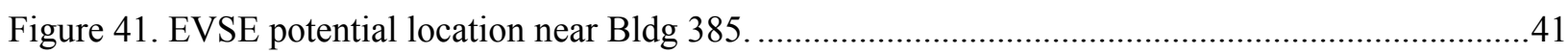

Figure 42. EVSE potential location near Bldg 2734 for various vehicles.............................................41

Figure 43. EVSE potential location near Bldg 243 for various vehicles....................................................42

Figure 44. EVSE potential location near Bldg 376 for Vehicle G41-1349G............................................42

Figure 45. EVSE potential location near Bldg 382 for Vehicle G10-0984N . ..........................................43

Figure 46. EVSE potential location near Bldg 960 for Vehicle G10-5286H........................................43

Figure 47. EVSE potential location near Bldg 2555 for Vehicle G42-0766L........................................43

Figure 48. EVSE potential location near Bldg 2556 for Vehicle G41-1137K........................................44

Figure 49. EVSE potential location near Bldg 2699 for Vehicle G41-4334M. ......................................44

Figure 50. EVSE potential location near Bldg 2704 for Vehicle G42-0335G.......................................45

Figure 51. EVSE potential location near Bldg 2737 for Vehicle G43-2287M. ......................................45

Figure 52. EVSE potential location near Bldg 2815 for Vehicle G43-0936K.......................................45

\section{TABLES}

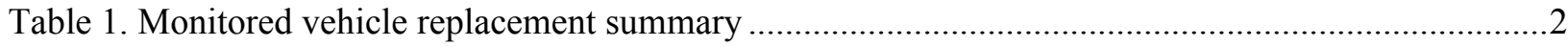

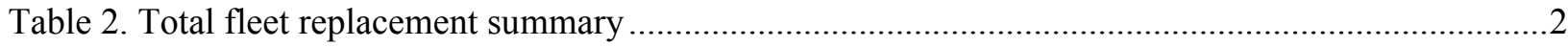

Table 3. Commands Group monitored vehicle replacements.................................................................18

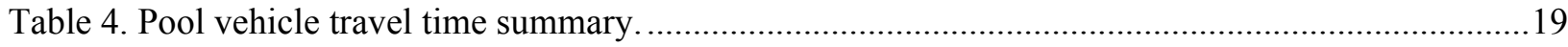

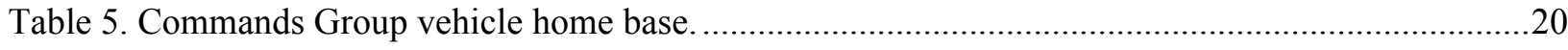




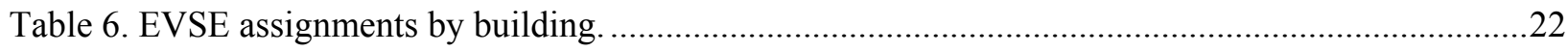

Table 7. Departments Group monitored vehicle replacements ...........................................................24

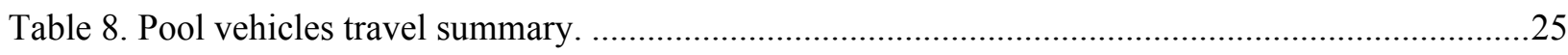

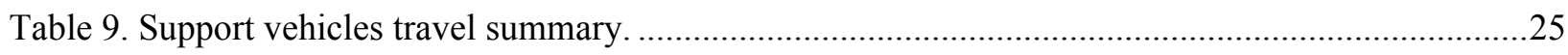

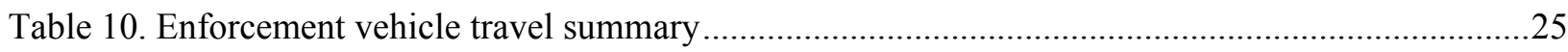

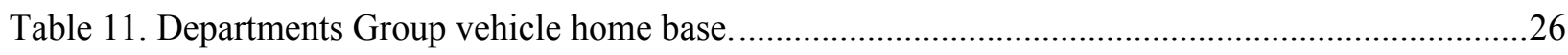

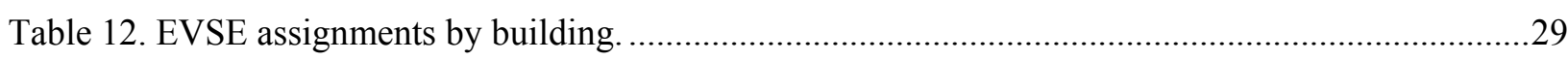

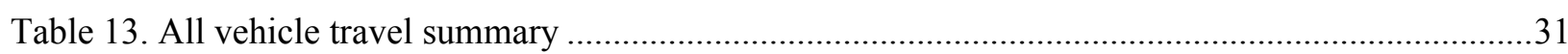




\section{ACRONYMS}

\begin{tabular}{ll} 
AC & Alternating current \\
BEA & Battelle Energy Alliance, LLC \\
BEV & battery electric vehicle \\
CD & Charge depletion \\
CS & Charge sustaining \\
DC & Direct current \\
EPA & U.S. Environmental Protection Agency \\
EVSE & electric vehicle supply equipment \\
GHG & greenhouse gas emissions \\
GSA & General Services Administration \\
ICE & internal combustion engine \\
INL & Idaho National Laboratory \\
Intertek & Intertek Testing Services, North America \\
NAVFAC & Naval Facilities Engineering Command \\
NASWI & Naval Air Station Whidbey Island \\
OEM & original equipment manufacturer \\
PEV & plug-in electric vehicle (includes BEVs and PHEVs, but not hybrid electric vehicles) \\
PHEV & plug-in hybrid electric vehicle \\
POV & privately owned vehicle \\
SPIDERS & Smart Power Infrastructure Demonstration for Energy Reliability and Security \\
SUV & sports utility vehicle \\
V2G & vehicle to grid \\
\hline
\end{tabular}




\section{Assessment of Charging Infrastructure for Plug-in Electric Vehicles At Naval Air Station Whidbey Island: Task 3 \\ 1. INTRODUCTION}

The U.S. Department of Energy and the U.S. Department of Defense signed a memorandum of understanding on July 22, 2010 for strengthening the coordination of efforts to enhance national energy security and to demonstrate federal government leadership in transitioning the U.S. to a low-carbon economy. The memorandum of understanding included efforts in the areas of energy efficiency, fossil fuels, alternative fuels, efficient transportation technologies and fueling infrastructure, grid security, smart grid, and energy storage.

In support of the memorandum of understanding, the Idaho National Laboratory with funding provided by the U.S. Department of Energy's Vehicle Technologies Office and Federal Energy Management Program, contracted with Intertek Testing Services, North America (Intertek) to conduct several U.S. Department of Defense base studies to identify potential transportation systems that are strong candidates for introduction or expansion of plug-in electric vehicles (PEVs). Intertek previously has conducted similar fleet, city, state, and countrywide studies using the micro-climate assessment process, which consists of the following four main tasks:

- Task 1: Conduct a non-tactical vehicle fleet assessment

- Task 2: Select vehicles for mission and fleet characterizations

- Task 3: Perform detailed assessment of selected vehicles and charging infrastructure needs

- Task 4: Provide implementation approach for PEV and charging infrastructure

Assessment of the potential for replacing Naval Air Station Whidbey Island (NASWI) fleet vehicles with PEVs starts with an assessment of the fleet vehicles' missions and vehicle characteristics. This assessment was conducted through review of NASWI records, as well as communications with NASWI personnel. The Task 1 report, titled, Assessment of Data and Survey Results for Naval Air Station Whidbey Island, dated January 2015, provided a summary and fleet assessment.

PEVs generally are classified into two vehicle types: battery electric vehicles (BEVs) and plug-in hybrid electric vehicles (PHEVs). A BEV contains an onboard battery that provides all motive power. PHEVs also have an onboard battery that provides some motive power by an onboard battery that is supplemented by another power source (such as a gasoline engine). Collectively, BEVs and PHEVs are PEVs.

The Task 1 effort led to identification of fleet vehicles that appear to be good candidates for replacement by PEVs. The Task 2 report, titled, Identification of Naval Air Station Whidbey Island Vehicles for Installation of Data Loggers, dated March 2015, identified the 60 vehicles within the candidate groups for further monitoring and analysis through addition of vehicle data loggers. The data loggers were installed and data collected on the use of these selected vehicles. The Task 3 report, titled, Utilization Assessment of Target Electrification Vehicles at Naval Air Station Whidbey Island, dated May 2015, provided a summary and details of the data collection for the monitored vehicles and extrapolated that to the entire non-tactical fleet of vehicles at NASWI. This report assesses the electric vehiclecharging infrastructure necessary to support the recommended electric vehicle replacements.

The infrastructure recommendations depend upon the type of PEV to be charged, time available for charging, locations of typical vehicle parking, fleet management attention, and electrical power availability. Other considerations such as providing charging opportunities for assigned military, base 
employees, or visitors may be of interest. While vehicle-to-grid (V2G) power transfer capabilities are currently under study at military facilities, this topic will be discussed but not evaluated as part of this report.

\section{FLEET VEHICLE PLUG-IN ELECTRIC VEHICLE RECOMMENDATIONS}

NASWI identified 60 vehicles for further study that was completed and reported in the Task 3 Vehicle Utilization report. In summary, this subset of vehicles contains nineteen sedans, nine minivans, three sports utility vehicles (SUV), eighteen pickup trucks, two cargo vans, and nine passenger vans. This distribution is intended to be representative of the entire non-tactical fleet.

PEVs currently commercially available cannot replace certain vehicles and missions, such as those requiring heavy-duty trucks, passenger vans, and specialty usage vehicles. However, the Task 3 vehicle utilization report suggested that based upon the data collected for the monitored vehicles, the 60 -vehicle fleet subset could possibly consist of 32 BEVs, and 28 PHEVs. Table 1 shows the replacement summary.

Table 1. Monitored vehicle replacement summary

\begin{tabular}{|c|c|c|c|c|c|c|c|c|c|}
\hline & $\begin{array}{c}\text { Sedan - } \\
\text { Compact }\end{array}$ & $\begin{array}{c}\text { Sedan - } \\
\text { Midsize }\end{array}$ & $\begin{array}{c}\text { Sedan- } \\
\text { Large }\end{array}$ & Minivan & SUV & $\begin{array}{c}\text { Van } \\
\text { Cargo }\end{array}$ & $\begin{array}{c}\text { Van } \\
\text { Pass }\end{array}$ & Pickup & Total \\
\hline ICE & - & - & - & - & - & - & - & - & 0 \\
\hline BEV & 2 & 3 & 0 & 7 & 1 & 2 & 5 & 12 & 32 \\
\hline PHEV & 3 & 8 & 3 & 2 & 2 & 0 & 4 & 6 & 28 \\
\hline Total & 5 & 11 & 3 & 9 & 3 & 2 & 9 & 18 & 60 \\
\hline
\end{tabular}

The monitored vehicles represent 60 vehicles of 175 on-road rated vehicles in these represented fleets. Assuming that the balance of these fleets operate in a manner similar to those monitored and without consideration of specific cargo or other mission requirements not previously identified, Intertek suggests the total fleet composition could consist of 92 BEVs, and 83 PHEVs. Table 2 shows this replacement summary.

Table 2. Total fleet replacement summary

\begin{tabular}{|c|c|c|c|c|c|c|c|c|c|}
\hline & $\begin{array}{c}\text { Sedan - } \\
\text { Compact }\end{array}$ & $\begin{array}{c}\text { Sedan - } \\
\text { Midsize }\end{array}$ & $\begin{array}{c}\text { Sedan- } \\
\text { Large }\end{array}$ & Minivan & SUV & $\begin{array}{c}\text { Van } \\
\text { Cargo }\end{array}$ & $\begin{array}{c}\text { Van } \\
\text { Pass }\end{array}$ & Pickup & Total \\
\hline ICE & - & - & - & - & - & - & - & - & 0 \\
\hline BEV & 1 & 5 & 4 & 7 & 1 & 37 & 6 & 31 & 92 \\
\hline PHEV & 4 & 15 & 6 & 4 & 12 & 17 & 13 & 12 & 83 \\
\hline Total & 5 & 20 & 10 & 11 & 13 & 54 & 19 & 43 & 175 \\
\hline
\end{tabular}

The sixty vehicles monitored in the study at NASWI belonging to Tennant Commands (Commands) and Naval Air Station departments and divisions (Departments). PEV replacements are separated by these groups, as are the recommendations for the total 175 vehicle replacements. Specific analysis by fleet group follows in Section 4. Figure 1 illustrates the final suggested vehicle summary. 


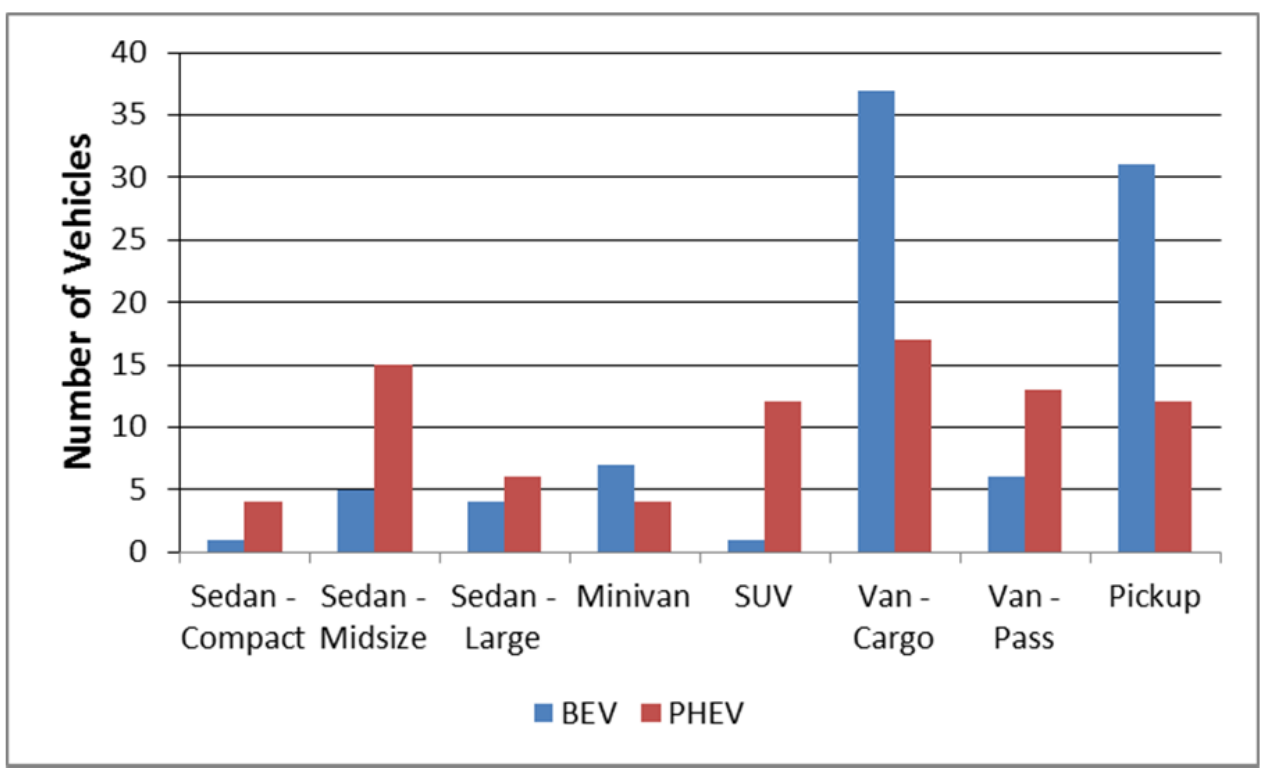

Figure 1. PEV type distribution for all vehicles.

\section{PLUG-IN ELECTRIC VEHICLE CHARGING}

Refueling electric vehicles presents some challenges and some opportunities not encountered when refueling petroleum-fueled vehicles. Recharging the battery of a PHEV follows the same methodology as that for BEVs. This section provides basic information on recharging PEVs.

\subsection{Electric Vehicle Supply Equipment Design}

\subsubsection{Charging Components}

Electric vehicle supply equipment (EVSE) stations deliver electric power from the electric grid to the applicable charge port on the vehicle. Figure 2 illustrates the primary components of a typical EVSE, which in the figure is an alternating current (AC) Level 2.

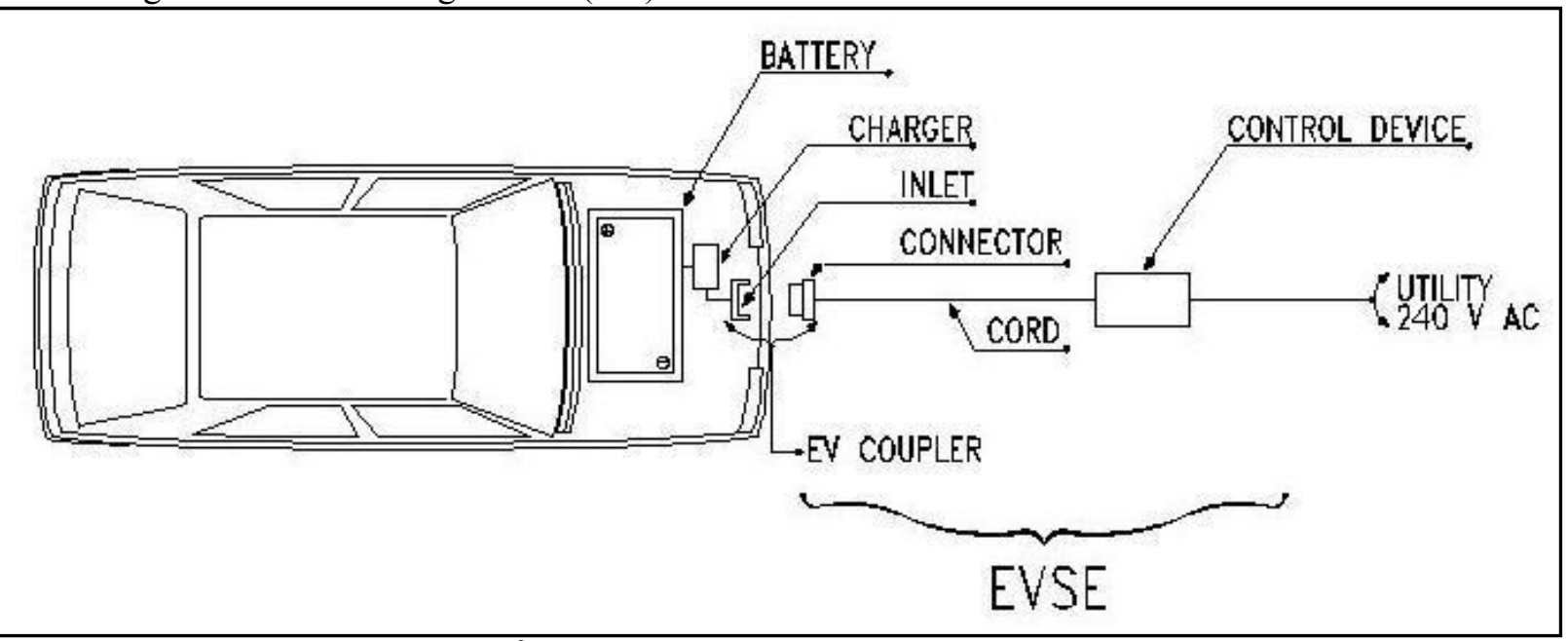

Figure 2. AC Level 2 charging diagram ${ }^{2}$

\footnotetext{
${ }^{2}$ http://avt.inl.gov/pdf/EVProj/EVChrgInfraDeployGuidelinesPhoenixVer3.2.pdf [accessed March 7, 2015]
} 
The electricity grid delivers alternating current (AC) to the charging location. The conversion from AC to direct current (DC) electricity is necessary for battery charging to occur either on or off board the vehicle. Further explanation of the different EVSE configurations follows in the next section. For on board conversion, AC current flows through the PEV inlet to the onboard charger. The charger converts $\mathrm{AC}$ to the DC current required to charge the battery. A connector attached to the EVSE inserts into a PEV inlet to establish an electrical connection to the PEV for charging and information/data exchange. Offboard conversion, also known as DC charging, proceeds in a similar manner except that the AC to DC conversion occurs in a charger that is off board the vehicle and, thus, bypasses any on board charger. For both AC and DC charging, the PEV's battery management system on board the vehicle controls the battery rate of charge, among other functions. All current PEVs have an onboard charger; some BEVs (but no PHEVs currently) accommodate DC charging.

\subsubsection{Charging Configurations and Ratings}

The Society of Automotive Engineers (SAE) standardized the requirements, configurations, and equipment followed by most PEV suppliers in the United States in the J1772 Standard. Figure 3 summarizes these attributes and the estimated recharge times. Actual recharge times depend on the on board equipment, including the charger, battery, and battery management system.

\begin{tabular}{|c|c|c|c|}
\hline \multicolumn{4}{|c|}{ SAE Charging Configurations and Ratings Terminology } \\
\hline \multirow{5}{*}{$\begin{array}{l}\text { AC level } 1 \\
\text { (SAE J1772 }\end{array}$} & PEV includes on-board charger & \multirow[t]{5}{*}{ *DC Level 1} & EVSE includes an off-board charger \\
\hline & $\begin{array}{l}120 \mathrm{~V}, 1.4 \mathrm{~kW} @ 12 \mathrm{amp} \\
120 \mathrm{~V}, 1.9 \mathrm{~kW} @ 16 \mathrm{amp} \\
\end{array}$ & & $200-450 \mathrm{~V} \mathrm{DC}$, up to $36 \mathrm{~kW}(80 \mathrm{~A})$ \\
\hline & Est. charge time: & & Est. charge time ( $20 \mathrm{~kW}$ off-board charger): \\
\hline & PHEV: 7 hrs (SOC* $-0 \%$ to full) & & PHEV: $22 \mathrm{~min}$. (SOC* $-0 \%$ to $80 \% 6)$ \\
\hline & BEV: $17 \mathrm{hrs}$ (SOC $-20 \%$ to full) & & BEV: 1.2 hrs. (SOC $-20 \%$ to $100 \%)$ \\
\hline \multirow[t]{11}{*}{$\begin{array}{l}\text { AC level } 2 \\
\text { (SAE J1772 }\end{array}$} & $\begin{array}{l}\text { PEV includes on-board charger (see below for different } \\
\text { types) }\end{array}$ & \multirow[t]{6}{*}{ *DC Level 2} & EVSE includes an off-board charger \\
\hline & $240 \mathrm{~V}$, up to $19.2 \mathrm{~kW}(80 \mathrm{~A})$ & & $200-450 \mathrm{~V} \mathrm{DC}$, up to $90 \mathrm{~kW}(200 \mathrm{~A})$ \\
\hline & Est. charge time for $3.3 \mathrm{~kW}$ on-board charger & & Est. charge time ( $45 \mathrm{~kW}$ off-board charger): \\
\hline & PEV: 3 hrs (SOC* $-0 \%$ to full) & & PHEV: $10 \mathrm{~min}$. (SOC* $-0 \%$ to $80 \%$ ) \\
\hline & BEV: 7 hrs (SOC $-20 \%$ to full) & & BEV: $20 \mathrm{~min} .(\mathrm{SOC}-20 \%$ to $80 \%)$ \\
\hline & Est. charge time for $7 \mathrm{~kW}$ on-board charger & & \\
\hline & PEV: 1.5 hrs (SOC* $-0 \%$ to full) & \multirow[t]{6}{*}{ *DC Level 3 (TBD) } & EVSE includes an off-board charger \\
\hline & BEV: 3.5 hrs (SOC $-20 \%$ to full) & & $200-600 \mathrm{~V} \mathrm{DC}$ (proposed) up to $240 \mathrm{~kW}(400 \mathrm{~A})$ \\
\hline & Est. charge time for $20 \mathrm{~kW}$ on-board charger & & Est. charge time ( $45 \mathrm{~kW}$ off-board charger): \\
\hline & PEV: $22 \mathrm{~min}$. (SOC* -09 to full) & & BEV (only): $<10 \mathrm{~min}$. (SOC ${ }^{*}-0 \%$ to 8096 ) \\
\hline & BEV: $1.2 \mathrm{hrs}$ (SOC $-20 \%$ to full) & & \\
\hline •AC Level 3 (TBD) & $>20 \mathrm{~kW}$, single phase and 3 phase & & \\
\hline \multicolumn{4}{|c|}{$\begin{array}{l}\text { "Not finalized } \\
\text { Voltages are nominal configuration voltages, not coupler ratings } \\
\text { Rated Power is at nominal configuration operating voltage and coupler rated current } \\
\text { Ideal charge times assume } 90 \% \text { efficient chargers, } 150 \mathrm{~W} \text { to } 12 \mathrm{~V} \text { loads and no balancing of Traction Battery Pack }\end{array}$} \\
\hline \multicolumn{4}{|r|}{ one hour) will also stop at $80 \%$ SOC instead of } \\
\hline
\end{tabular}

Figure 3. Society of Automotive Engineers charging configurations and ratings terminology ${ }^{3}$

\footnotetext{
${ }^{3}$ http://www.sae.org/smartgrid/chargingspeeds.pdf [accessed March 7, 2015]
} 
PEVs are typically sold with an ACL1 cordset included. This cordset is generally intended to be used in emergencies when the vehicle is away from installed EVSE and any 120-volt electrical outlet may be available for recharge. A typical cordset is shown in Figure 4.

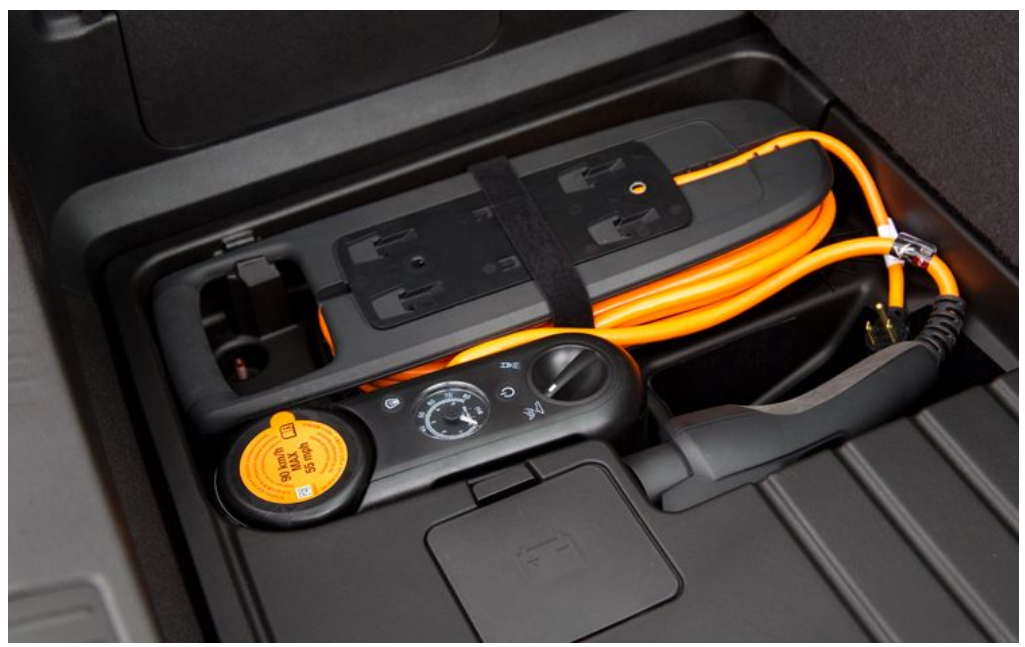

Figure 4. Chevrolet Volt ACL1 cordset ${ }^{4}$.

AC recharging capabilities found in the public arena more typically are AC Level 2. Figure 5 depicts a typical J1772-compliant inlet and connector for both AC Levels 1 and 2.

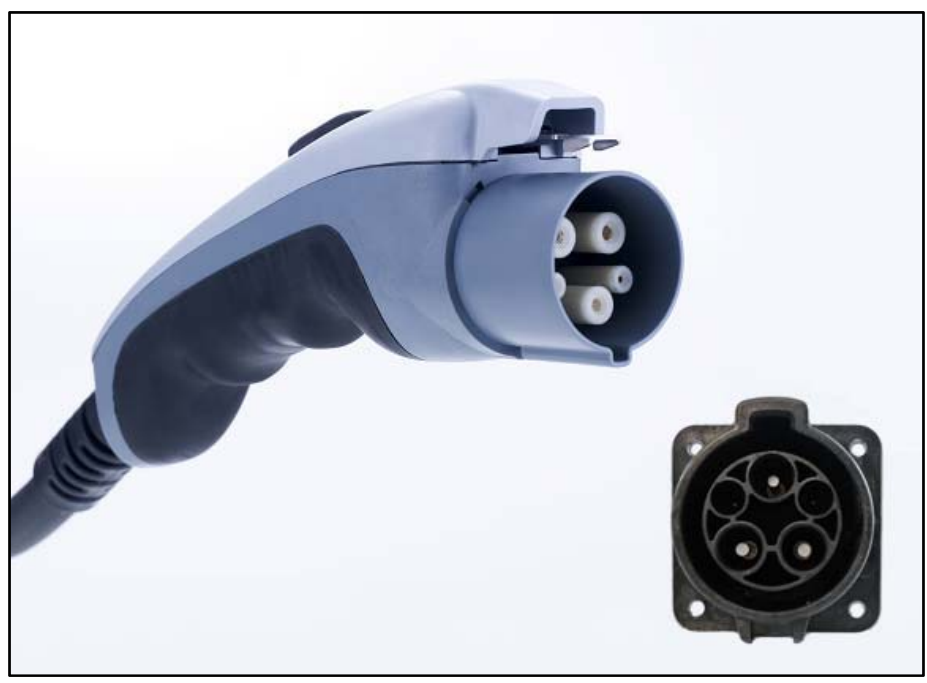

Figure 5. J1772 connector and inlet ${ }^{5}$

The J1772 standard also identifies requirements for DC charging. For PEVs that accept both AC and DC inputs, the SAE approved a single connector and inlet design. Figure 6 shows this connector, which is colloquially known as the J1772 "combo connector".

Some PEVs delivered in the United States prior to the approval of the J1772 standard for DC charging employed the CHAdeMO standard for connector and inlet design. Figure 7 shows this

\footnotetext{
${ }^{4}$ www.pluginamerica.org

${ }^{5}$ http://carstations.com/types/j09 [accessed March 7, 2015]
} 
connector. EVSE units that are either J1772-compliant or CHAdeMO-compliant are both known as DC fast chargers (DCFCs).
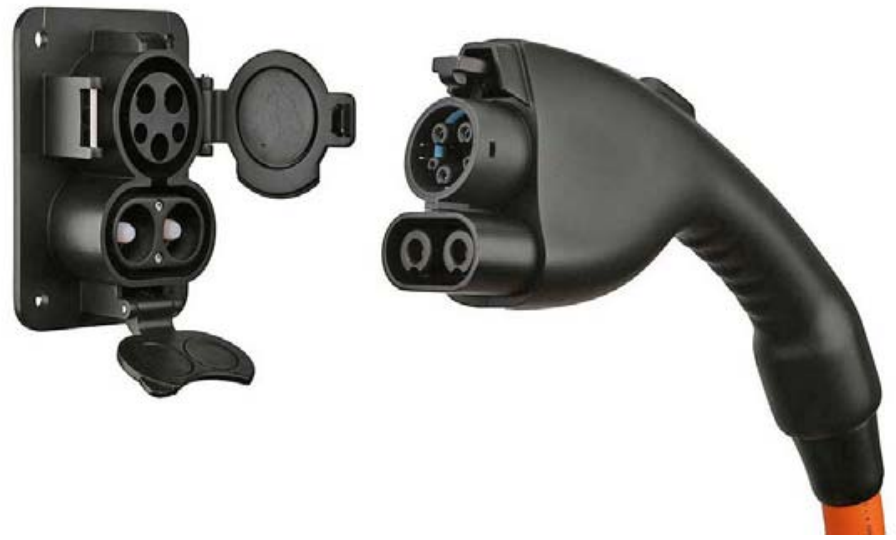

Figure 6. J1772-compliant combo connector ${ }^{6}$

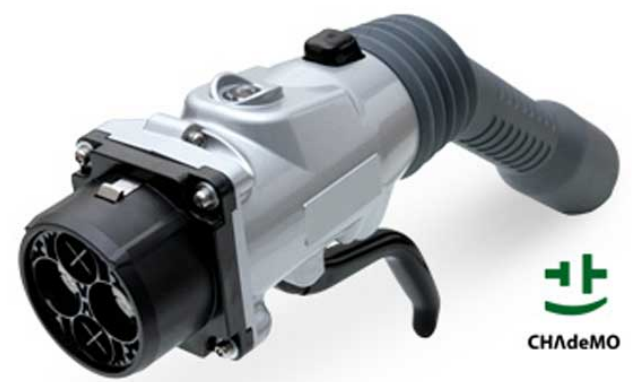

Figure 7. CHAdeMO-compliant connector ${ }^{7}$

Tesla Motors has developed a charge connector unique to their vehicles. An adapter is provided to allow the use of public charging stations using the J1772 standard for AC Level 2. Tesla has also developed a network of "superchargers" for use with their vehicles that provide up to $120 \mathrm{~kW}$ power. ${ }^{8}$ The presence of the two separate standards for DC charging (along with the unique Tesla connector) presents challenges for vehicle owners to ensure that the EVSE accessed provides the appropriate connector for their vehicle inlet. Not all PEV suppliers include DC charging options. BEV suppliers more typically provide DC inlets than PHEV suppliers do, because the rapid recharging provides opportunities for expanded vehicle range with minimal operator wait times. PHEV operators can rely on the gasoline drive in the event they deplete the vehicle's battery; at present, no PHEV on the market or near commercialization has DC charging capability (although the Mitsubishi Outlander PHEV is rumored to be offering DC charging capability as an option). It is noted that DC Level 1 and DC Level 2 charging are commonly combined and labeled "DC fast charging."

\footnotetext{
${ }^{6}$ http://www.zemotoring.com/news/2012/10/sae-standardizes-j1772-fast-dc-charging-up-to-100-kw [accessed March 7, 2015]

${ }^{7}$ https://radio.azpm.org/p/azspot/2012/5/10/1632-electric-cars/ [accessed March 7, 2015]

${ }^{8}$ http://www.teslamotors.com/supercharger [accessed March 7, 2015]
} 
Because the battery of a BEV is typically much larger than that of a PHEV, recharge times are longer (see Figure 3). BEVs that see daily mileage near the limits of the advertised range do better when recharged using AC Level 2 EVSE or DCFC, because AC Level 1 recharge times are usually extensive. PHEVs, on the other hand, generally can use AC Level 1 EVSE for overnight charging to ensure a fully charged battery at the start of daily use. AC Level 2 EVSE units provide greater range in the shortest amount of time when intermediate or opportunity charging. DCFC provides the fastest recharge capability for those vehicles equipped with DCFC inlets.

\subsection{Electric Vehicle Supply Equipment Stations}

Figure 8 provides an example of a public AC Level 2 EVSE unit. ${ }^{9}$ The unit shown is known as a "pedestal" or "bollard" unit because it is a self-standing unit. Wall or post-mounted models are also available by several suppliers.

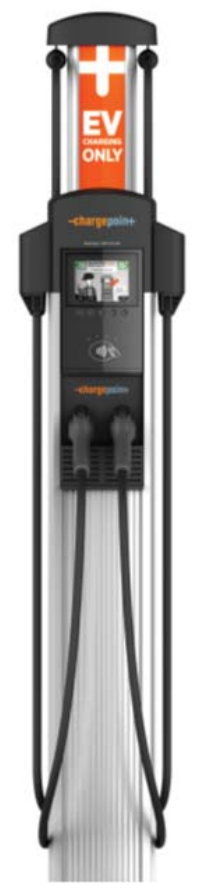

Figure 8. AC Level 2 unit.

AC Level 1 EVSE is available in permanently installed models and is similar in appearance to that of AC Level 2 units.

AC Level 2 charging is the predominant rating of publicly accessible EVSE because of its wide acceptance by auto manufacturers and faster recharge times than AC Level 1. Purchase and installation costs are more manageable than DCFCs and less space is required. There are several manufacturers of AC Level 2 equipment and the agency should review brands for comparison purposes.

DCFCs also are available from several manufacturers. Figure 9 illustrates one such charger. ${ }^{10}$ This particular unit uses the CHAdeMO connector standard.

\footnotetext{
${ }^{9}$ http://www.chargepoint.com/files/73-001061-01-2_BR-CT4000-01.pdf [accessed March 7, 2015]

${ }^{10} \mathrm{http} / /$ evsolutions.avinc.com/products/public_charging/public_charging_b [Accessed March 7, 2015]
} 
Figure 9. Public DCFC.

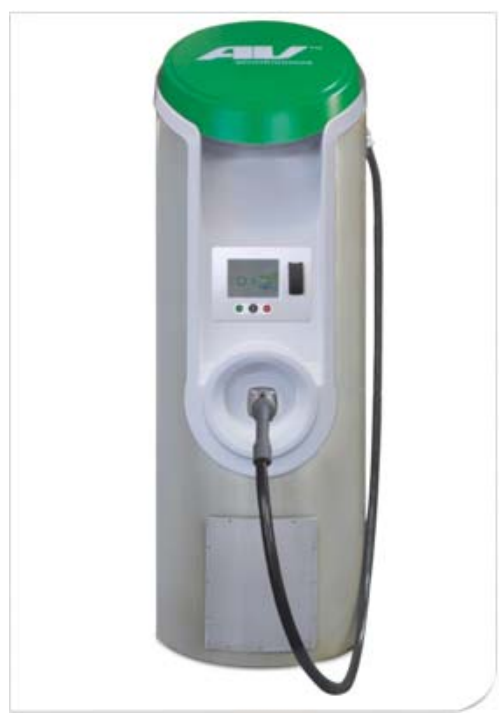

In general, installation costs are higher for the DCFC because of the higher voltage requirements and the inclusion of the AC to DC converter and other safety and design features. Installation costs for both types are highly dependent on site characteristics such as distance to the nearest power source, asphalt or concrete cutting and repair, conduit requirements, and payment systems, if any.

Payment and equipment control systems included by some suppliers provide the potential for use by privately owned vehicles for a fee, but allow agency fleet vehicle use without direct payment. These systems allow for accurate record keeping of vehicle charging requirements.

EVSE designs also include dual port or multi-port options that feature charging vehicles simultaneously or sequentially depending on design.

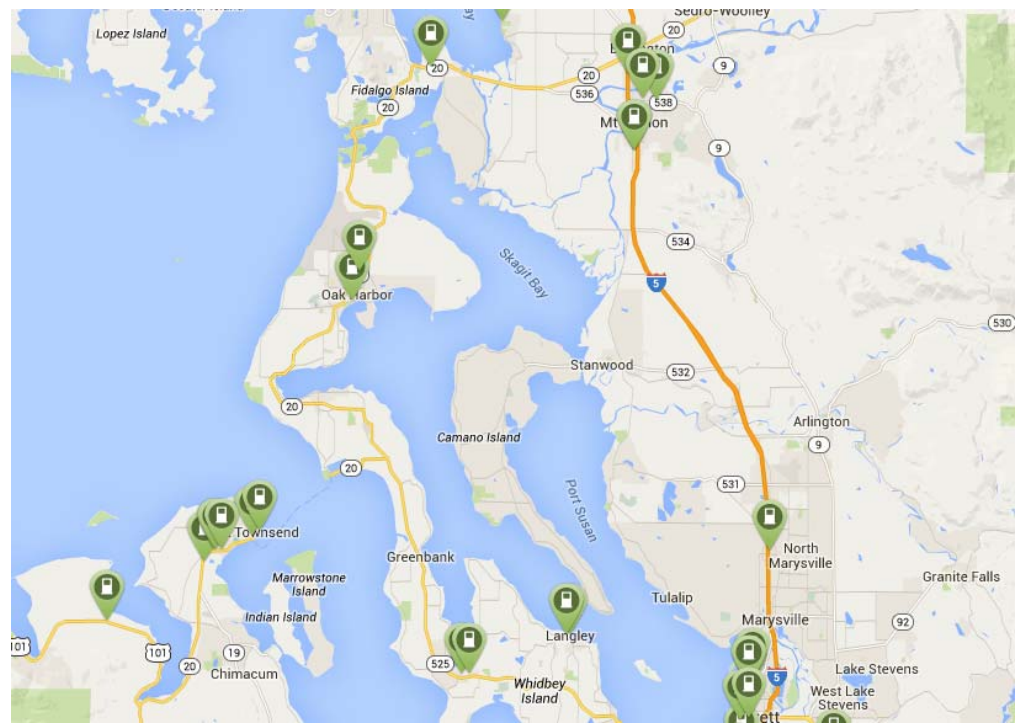

Figure 10. Public EVSE in NASWI Region. ${ }^{11}$

\footnotetext{
${ }^{11}$ http://www.plugshare.com/ [accessed March 6, 2015]
} 
At times, fleet vehicles obtain benefit from using public charging infrastructure. Figure 10 displays the availability of public charging at the time of this writing for the NASWI area. The greencolored sites are AC sites indicating either AC Level 1 or Level 2 public locations.

Not shown is the high density of publicly accessible EVSE in the greater Seattle area that reflects the high local interest and adoption of PEVs. It also illustrates the high potential for adoption of PEVs by NASWI attached military, civilian employees, and local contractors who may be interested in PEV charging locations.

\subsection{EVSE Installation}

Numerous factors must be considered before installation of the EVSE can occur. The Electric Vehicle Charging Infrastructure Deployment Guidelines document provided by The EV Project ${ }^{12}$ provides specific information related to commercial fleet charging. While military bases may not be required to address all these considerations, this information may be of use in site selection. Figure 11 provides a flowchart of installation considerations for fleet applications.

Specific considerations include:

1. AC Level 1, Level 2 or DCFC units:

The Task 3 Vehicle Utilization report provided recommendations related to the power level needs of the associated vehicles. This will be discussed in detail in Section 4.

2. Proximity to power supply connections:

Installation of the EVSE in a commercial facility typically will consist of installing new dedicated branch circuits from the central meter distribution panel to a Level 2 EVSE. In a commercial fleet, there are typically many such EVSE units in adjacent parking stalls. Proximity to the electrical service is an important factor in locating this parking area. The length of the circuit run and the number of EVSE units will have a significant impact on the cost. $^{13}$

In addition, the cost for installation will be influenced by whether concrete or asphalt cuts will be required along with the restoration of these surfaces. Conduit runs through landscaped areas will be less expensive.

Consideration should also be given to the potential for adding PEVs in the future. Installing additional conduit and conductors to feed future EVSE units reduces future costs while not adding significantly to current costs.

\section{Parking space restrictions:}

Determination should be made in advance whether the EVSE parking space will be restricted to PEVs only. If so, the dedication of these spaces may reduce the availability of spaces for conventional vehicles. This may affect the functional needs of the existing operation. On the other hand, non-PEVs parking in charging locations will inhibit the recharge ability of the PEVs and may interfere with their mission. Intertek recommends dedicating charging stations to PEV charging only.

\footnotetext{
${ }^{12}$ http://avt.inl.gov/pdf/EVProj/EVChrgInfraDeployGuidelinesSanDiegoVer3.2.pdf [accessed March 7, 2015]

${ }^{13}$ ibid
} 


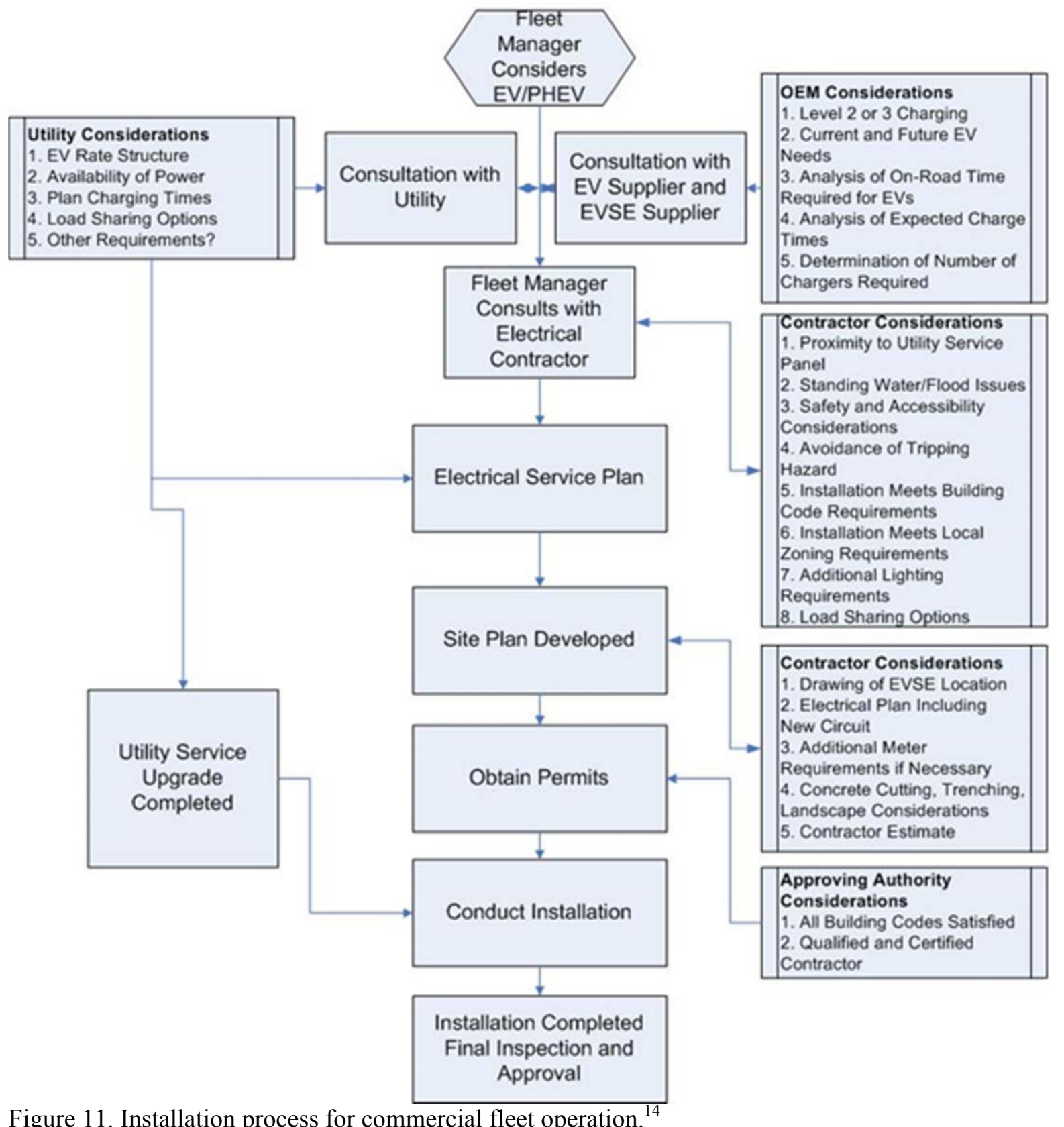

4. ADA considerations:

The Americans with Disabilities Act provides requirements on accessibility for many situations but does not directly address PEV charging stations. The EV Project provided recommendations to maximize compliance within the specific restraints and constraints of PEV operation. While

${ }^{14}$ ibid 
the exact requirements may not be applicable to military bases, the recommendations may present best practices for NASWI consideration. ${ }^{15}$

5. Physical protection of the equipment:

Unless de-energized by the local disconnect, the EVSE is considered electrically energized equipment. Because it operates above 50 volts, Part 19 Electrical Safety of the Occupational Health and Safety (OHS) Regulation requires guarding live parts. EVSE may be positioned in a way that requires a physical barrier for its protection. Frequently, wheel stops, curbs, or bollards are utilized to provide physical protection.

6. Accessibility and Usage:

EVSE units will typically be powered from the electrical grid near the parking location. The base provides this electrical supply for the general use to carry out its mission on the base. The power delivered to fleet vehicles supports their DOD mission. However, federal restrictions do not allow the use of federally provided electrical power to be utilized by privately owned vehicles (POVs). For each EVSE installed then, the installing agency fleet must decide whether access to the EVSE will be restricted to fleet vehicle only or whether POVs will be allowed to utilize these units.

Restricting use to agency fleet vehicles can be accomplished by physically segregating the charging infrastructure to restricted parking locations that can be fenced and gated or by electronically segregating the usage by use of access control cards. Several EVSE suppliers provide access cards capable of allowing usage only by authorized cardholders.

Another option may allow for use by POV drivers using access cards that assess a fee for the charging opportunity. Under this option, the fleet drivers' cards allow access without the fee but the POV driver is charged the fee. The fee structure can be based upon the cost of electrical energy dispensed or by the amount of time connected. EVSE suppliers have options related to these structures. Fleets managers will need to monitor EVSE usage to insure their fleet vehicle have priority in charging to complete their mission.

7. Data Acquisition and Reporting:

It is likely that accounting for the electrical energy used for recharging vehicles will be required. Typically, the electrical energy supplied to a building is a facility cost but energy delivered to PEVs will be fuel costs. While the supply to the EVSE can be metered, Intertek suggests the use of "smart" EVSE capable of data collection and transmittal can be very useful to identify usage associated with specific vehicles and thus enhance fleet management.

8. Base Cyber Security:

Smart EVSE are likely to collect data locally in the EVSE or network of EVSE and transmit via internet or cellular communications to an off-site data center. Transmittal of the data back to the DOD facility may create issues with cyber security for the facility. Local transmittal to the appropriate fleet manager could be a benefit.

Figure 12 shows a fleet EVSE installation showing a significant number of stations with physical protection barriers. This site is not accessible to the public or for use by POVs.

\footnotetext{
${ }^{15}$ http://avt.inl.gov/pdf/EVProj/EVProjectAccessibilityAtPublicEVChargingLocations.pdf [accessed March 7, 2015]
} 


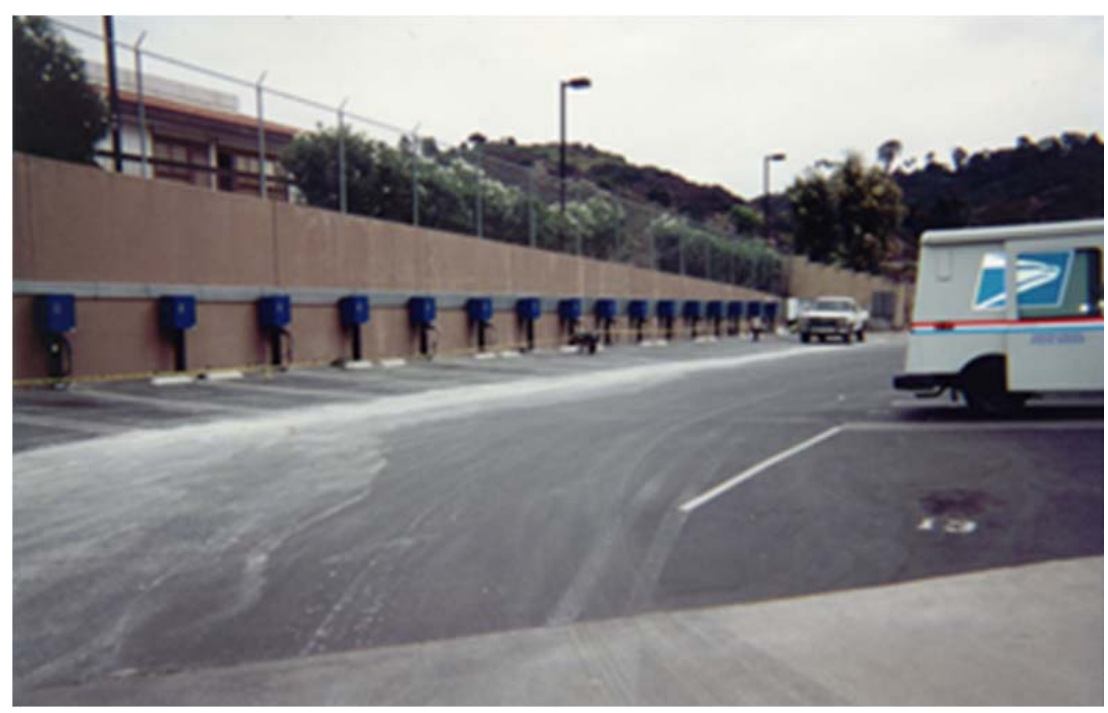

Figure 12. Typical fleet charging installation. ${ }^{16}$

EVSE installations intended for use by POVs may be required to follow other requirements. Figure 13 shows a public installation site complying with the ADA requirements. Note the accessible EVSE and parking location on the right.

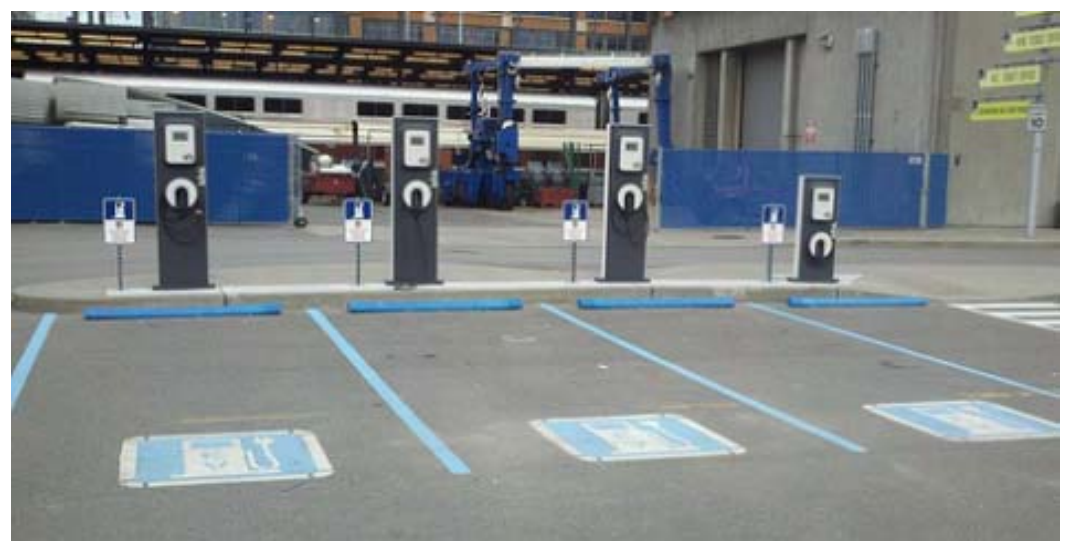

Figure 13. Publicly accessible EVSE. ${ }^{17}$

The General Services Administration (GSA) provides information and guides related to PEVs. The Installation Guide linked on their website provides specific installation instructions for the ChargePoint Networked Charging Station. ${ }^{18}$ These instructions apply once all the other site conditions have been resolved and provide the detailed systematic instruction for installing a ChargePoint brand EVSE.

\subsection{EVSE Advanced Design Considerations}

Several other charging topics may be of interest to NASWI in evaluating EVSE designs.

\footnotetext{
${ }^{16}$ http://avt.inl.gov/pdf/EVProj/EVChrgInfraDeployGuidelinesSanDiegoVer3.2.pdf [accessed March 7, 2015]

${ }^{17}$ https://www.flickr.com/photos/blinknetwork1/page3/ [accessed March 7, 2015]

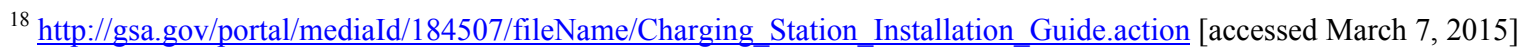




\subsubsection{Public Charging Access}

Figure 10 identifies the local public charging infrastructure in the NASWI region. The high local interest and adoption of PEVs supports this public infrastructure. For vehicles that travel off base, this infrastructure can provide a backup support for charging vehicles. Because PHEVs provide their own backup motive power, this feature may be more important for BEV drivers. NASWI may wish to consider obtaining access to these public chargers.

\subsubsection{Electric Utility Demand Charges}

Puget Sound Energy provides the electrical supply to NASWI. Puget Sound Energy reports a mix of its own generation and power provided by other sources. Electric rate schedule 49 for commercial or industrial customers includes electrical supply demand charges. The demand charge is billed at the highest measured demand for the month. Not only is the energy (kWh) consumed billed to NASWI but the power $(\mathrm{kW})$ delivered is also billed. PEV charging costs are impacted by this demand charge.

Figure 3 identifies the power requirements for EVSE charging. Up to $19.2 \mathrm{~kW}$ power is identified for AC Level 2. The power is controlled by the PEV's onboard battery management system and the capability of the onboard charger. These are typically rated at 3.6 or $7.2 \mathrm{~kW}$. The power required by DCFC is up to $90 \mathrm{~kW}$ but typical DCFC installed in the NASWI area deliver up to $60 \mathrm{~kW}$; again controlled by the vehicle's battery management system.

The Puget Sound Energy's demand charge for Schedule $49^{19}$ is $\$ 3.70 / \mathrm{kVa}$. There is a cost impact for the operation of both AC Level 2 and DCFC. The impact of DCFC is up to 8 times that of AC Level 2 although the simultaneous operation of AC Level 2 can have the same impact.

The electric vehicle industry is concerned with this impact especially for those EVSE installed in public locations by retailers. Methods are being tested to mitigate the impact of these demand charges. Options for AC Level 2 may include:

- Staggering the EVSE start times - This avoids all EVSE operating at high power at the same time,

- Shifting EVSE start times to evening and night hours - The highest usage by NASWI would typically occur during the daytime hours so the charging peak would not add to the non-charging peak.

- Limiting the power delivered by the EVSE - However, this also reduces the energy returned to the PEVs resulting in longer recharge times that defeats many of the benefits of fast charging.

- Augmenting EVSE supply power - Storage assisted recharging, discussed below, is one method to reduce the demand from the utility.

- Smart grid charging - Some EVSE are equipped with features including communications with the electric utility. Automatic controls can be set to reduce or curtail charging upon specific signals from the utility related to overall demand. Such features could also be integrated with the site power to assist in lowering charging demand.

All the features designed to mitigate power demand may affect the recharging of vehicles since the power is interrupted or curtained in response and thus would influence the actual power delivered to the PEV.

\subsubsection{Storage Assisted Recharging}

Storage assisted recharging utilizes a storage battery to augment power delivered from the electric grid to provide the PEV recharge energy. Figure 14 provides a conceptual sketch of such a system. This

\footnotetext{
${ }^{19} \mathrm{http}: / /$ pse.com/aboutpse/Rates/Documents/summ_elec_prices_2014_01_01.pdf [accessed March 7, 2015]
} 
system may be available for AC Level 2 or DCFC charging stations. The local storage battery is discharged during the PEV charging operation at a rate that limits the power demand from the electric utility and thus limits the demand charges. The battery may be recharged using renewable energy sources (i.e. wind or solar) or directly from the grid during non-charging operations. System design will depend on the number and ratings of the EVSE as well as the availability of renewable energy sources and anticipated PEV charging requirements.

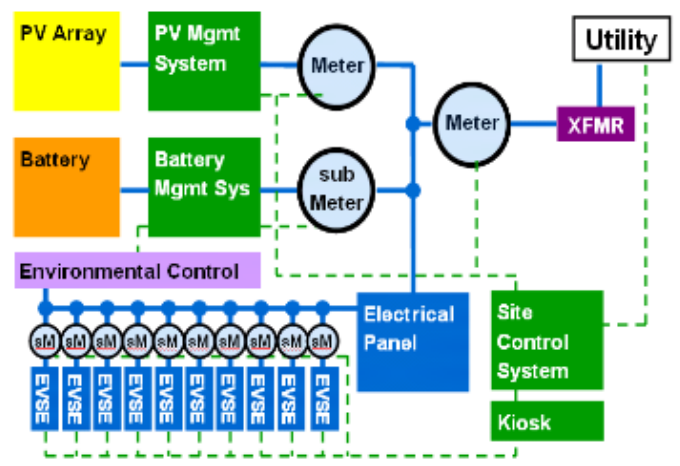

\begin{tabular}{|c|c|}
\hline \multicolumn{2}{|c|}{ Sample System Parameters } \\
\hline \multicolumn{2}{|c|}{ (values are per parking space) } \\
\hline Solar PV Array & $2 \mathrm{~kW}$ \\
\hline Battery Storage & $5 \mathrm{~kW} / 5 \mathrm{kWhr}$ \\
\hline Vehicle Charging & $240 \mathrm{~V}, 32 \mathrm{~A}(7.68 \mathrm{~kW})$ \\
\hline
\end{tabular}

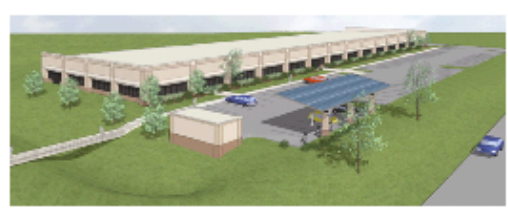

Figure 14. Conceptual design storage assisted recharging. ${ }^{20}$

\subsubsection{Vehicle to Grid}

Vehicle to Grid (V2G) technology is the transfer of energy from the PEVs onboard storage battery to the electrical grid. Amongst the uses for this electrical energy are:

- Installation peak power shaving

- Frequency regulation/power regulation

- Voltage and VAR Optimization

- Voltage regulation

- Spinning reserve functionality

- Emergency back-up power

- Installation microgrid connectivity and support $\mathrm{t}^{21}$

Figure 15 illustrates some of the opportunities for V2G benefits.

${ }^{20}$ http://et.epri.com/Charging_Vehicles_with_Solar_Power_and_Energy_Storage_2011.html [accessed March 7, 2015]

${ }^{21}$ Statement of Work for Grid Services Demonstration - US Army-TARDEC_NAC April 2, 2012 


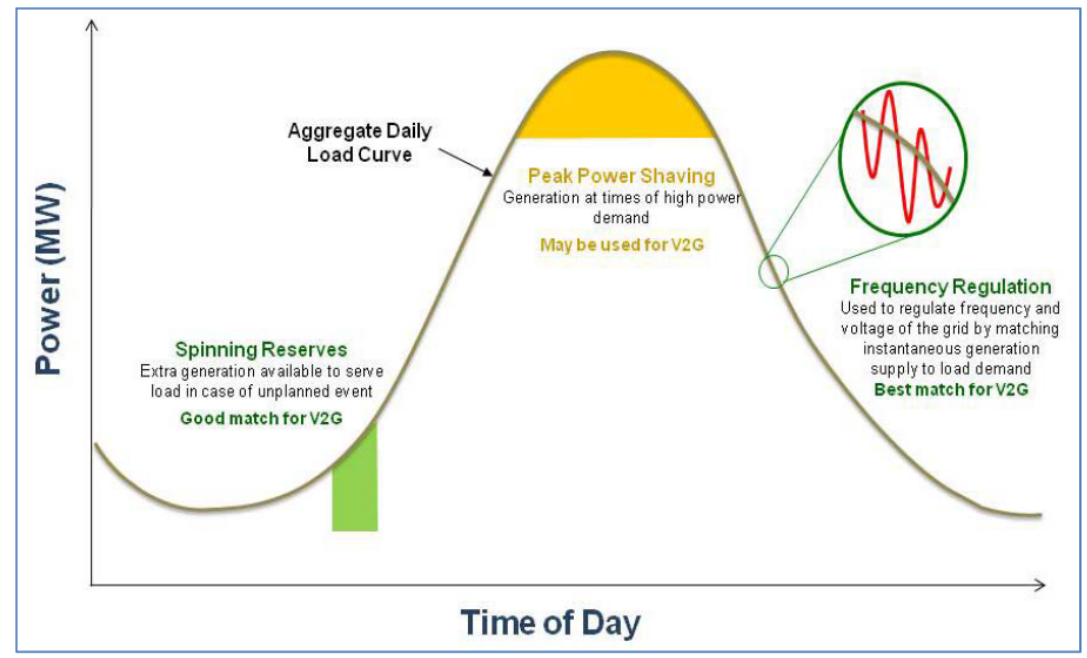

Figure $15 . \mathrm{V} 2 \mathrm{G}$ applications $\mathrm{s}^{22}$

The electric load for the electric utility typically follows the aggregate daily load curve shown in Figure 15 where peak power demands occur during the daylight hours and low power demand occurs in the evening and night hours. Utilities match this load by adding or removing generators in their supply. Typically, an electric utility will "base load" generation with their least cost generation capabilities (such as hydroelectric and nuclear). "Spinning reserve" generation is operational and ready to take grid load as the demand increases and "peaking" generation covers the peak daily demands with generators that are easier to start and stop (gas) and that are typically more costly to run. If sufficient power is available from the storage batteries of the PEVs and that energy can be transferred to the grid, the utility may be able to avoid generation. That saves utility generation costs for which the utility may be disposed to compensate the V2G supplier. In a similar manner, electric utilities may be interested in the other potential benefits noted above.

U.S. Department of Defense is interested in V2G as a potential technology to provide financial support in the adoption of PEVs at Department of Defense facilities and for support of Department of Defense energy security in establishing the facility microgrid. The microgrid isolates the facility in the event of a sustained loss of utility supplied power and the facility provides its own generation for strategic and tactical missions.

The Smart Power Infrastructure Demonstration for Energy Reliability and Security (SPIDERS) program was initiated to address the energy security issues in several phases. Each phase increases the size and complexity of the microgrid as renewable energy sources and V2G are added. ${ }^{23}$

In order to support the bi-directional power flow from the vehicle, the vehicle must have the ability to transfer power from the onboard battery off-board to the EVSE. The EVSE must be able to accept the reverse power flow and supply to the local grid. Control systems must be in place to monitor and direct the process as well as safety and security systems in handling electrical energy and internet/communications systems.

\footnotetext{
22 ibid

${ }^{23}$ Smart Power Infrastructure Demonstration for Energy Reliability and Security presentation, USPACOM/USNORTHCOM, May 2012
} 
The V2G capabilities can be realized with either AC or DC charging equipment. A few DCFC can provide significant power (if the vehicle can support the battery discharge) and the aggregated energy from several AC Level 2 EVSE can also be effective.

V1G capabilities refer to the single-directional power control afforded by controlling the charge rate to the connected PEVs. Rather than reverse power to the grid, grid peak and frequency is controlled by regulating the power to the connected PEVs.

Should NASWI be interested in possessing V2G or V1G capability, several other design considerations need to be studied as well as selecting the supplier of the smart EVSE capable of providing the bi-directional services.

\section{FLEET VEHICLE CHARGING ANALYSIS}

Sixty vehicles were included in the study at NASWI assigned to Tenant Commands (Commands) or to NASWI divisions and departments (Departments). The specific requirements of each group suggest that these data be analyzed by group and by aggregating across all vehicles. The Task 3 Vehicle Utilization report provides recommendations for PEV substitution for ICE vehicles by these fleets. Several terms were defined in the data collection process and included in that report. Topics directly related to the vehicle-charging infrastructure are emphasized here followed by the analysis by fleet.

\subsection{Analysis Background}

Non-intrusive data loggers were installed into the monitored vehicle's on-board diagnostic port to collect and transmit the relevant data. Data consist of key-on events, key-off events, and position updates logged every minute while the vehicle is keyed-on.

From these data points, the following information was available for evaluation:

- Trip start and stop time and location

- Trip distance and duration

- Idle start time, location, and duration

- Stop start time, location, and duration

For charging analysis, the stop locations and durations are the most significant since most recharging will occur at the vehicle's home base.

It is important to define other terms used in the analysis. Figure 16 illustrates a vehicle outing, which is comprised of trips, stops, and idle events, that may occur over one day or several days. The following list provides a definition of these terms:

1. Outing: An outing is the combination of trips and stops that begin at the home base and includes all travel until the vehicle returns home.

2. Trip: A trip begins with a key-on event and ends with the next key-off event.

3. Vehicle stop: A vehicle stop includes a key-off/key-on event pair.

4. Idle time: Idle time is the amount of time a vehicle spends stationary after a key-on event when the vehicle is not moving for a period of 3 minutes or longer. 
5. Trip travel time: Trip travel time is the amount of time required to complete a trip, excluding stops but including idle time.

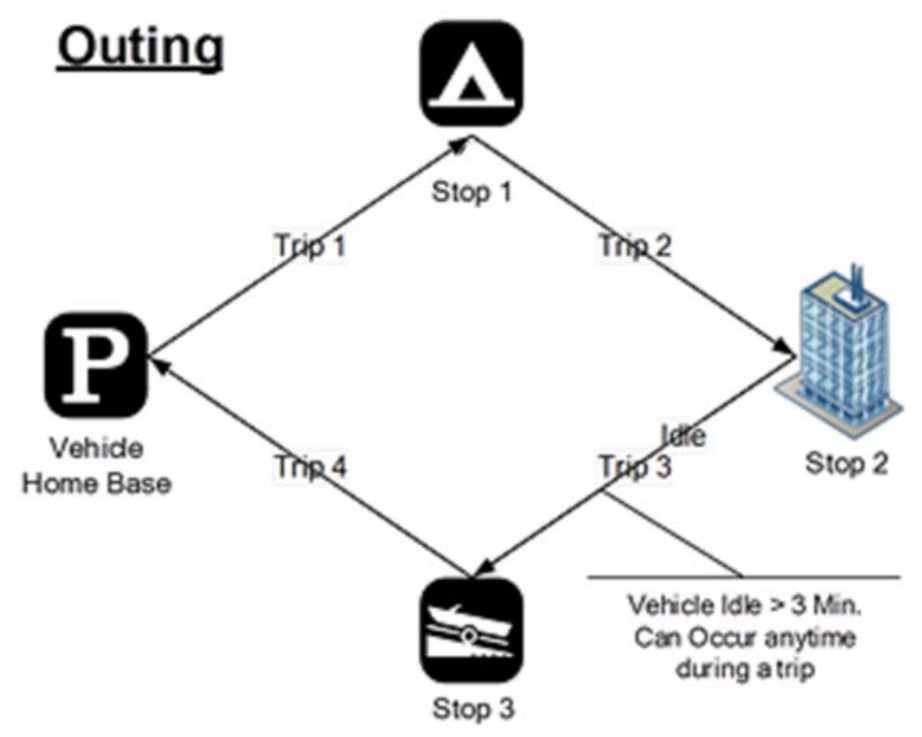

Figure 16. Vehicle outing.

The vehicle's mission influences the PEV recommendations. Based upon fleet information gathered, Intertek has established the following seven mission/vehicle categories for analysis. They are listed below and examples are depicted in Figure 17.

1. Pool vehicles: A pool vehicle is any automobile (other than the low speed vehicles identified below) manufactured primarily for use in passenger transportation, with not more than 10 passengers.

2. Enforcement vehicles: Vehicles specifically approved in an agency's appropriation act for use in apprehension, surveillance, police, or other law enforcement work. This category also includes site security vehicles, parking enforcement, and general use, but the vehicles are capable of requirements to support enforcement activities.

3. Support vehicles: Vehicles assigned to a specific work function or group to support the mission of that group. Vehicles are generally passenger vehicles or light-duty pickup trucks and may contain after-market modifications to support the mission.

2. Transport vehicles: Light, medium, or heavy-duty trucks used to transport an operator and tools or equipment of a non-specific design or nature. The vehicle's uses include repair, maintenance, or delivery.

3. Specialty vehicles: Vehicles designed to accommodate a specific purpose or mission (such as ambulances, mobile cranes, and handicap controls).

4. Shuttles/buses: Vehicles designed to carry more than 12 passengers and further outlined in 49 CFR 532.2.

5. LSV: Vehicles that are legally limited to roads with posted speed limits up to $45 \mathrm{mph}$ and that have a limited load-carrying capability. 


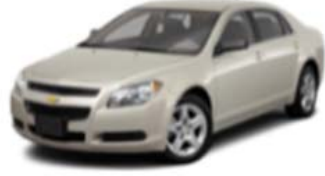

Pool Vehicle

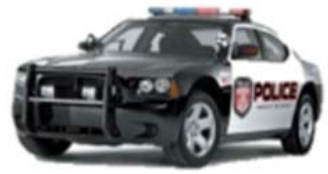

Enforcement Vehicle

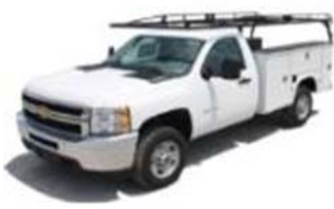

Support Vehicle

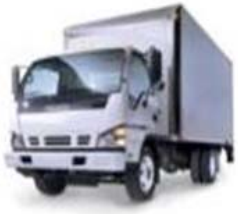

Transport Vehicle

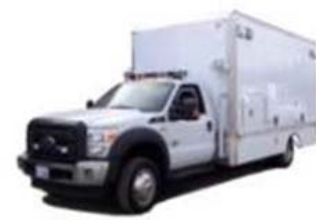

Specialty Vehicle

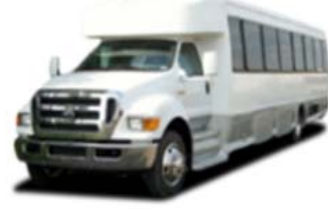

Shuttle / Bus

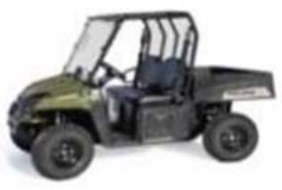

Low Speed

Vehicles

Figure 17. Vehicle missions.

\subsection{Plug-in Electric Vehicle Charging Analysis Results - Commands}

This section summarizes and aggregates the data collection for the Commands Group. The details of each vehicle monitored are included in Appendix B of the Task 3 Vehicle Utilization report.

\subsubsection{Commands Group Recommended Plug-in Electric Vehicles for Monitored Vehicles}

The Task 3 Vehicle Utilization report recommended replacement of the monitored vehicles with those identified in Table 3. See that report for vehicle analysis. All the vehicles in the group are support vehicles.

Table 3. Commands Group monitored vehicle replacements.

\begin{tabular}{|c|c|c|c|c|c|}
\hline \multicolumn{1}{|c|}{$\begin{array}{c}\text { Fleet } \\
\text { Vehicle Id }\end{array}$} & Make & Model & EPA Class & Replacement PEV & Mission \\
\hline G10-1138M & Chevrolet & Malibu & Sedan - Midsize & Ford Fusion & Support \\
\hline G10-1140M & Chevrolet & Malibu & Sedan - Midsize & Nissan Leaf & Support \\
\hline G10-3576L & Chevrolet & Malibu & Sedan - Midsize & Ford Fusion & Support \\
\hline G10-7547F & Dodge & Avenger & Sedan - Midsize & Nissan Leaf & Support \\
\hline G41-1136K & Dodge & Grd Caravan SE & Minivan & Nissan Leaf & Support \\
\hline G41-1139K & Dodge & Grd Caravan SE & Minivan & Honda Fit & Support \\
\hline G41-1140K & Dodge & Grd Caravan SE & Minivan & Honda Fit & Support \\
\hline G41-1155K & Dodge & Grd Caravan SE & Minivan & $\begin{array}{c}\text { Mitsubishi } \\
\text { Outlander }\end{array}$ & Support \\
\hline G41-1351G & Dodge & Dakota & Pickup & Toyota Rav4 & Support \\
\hline G41-2911M & Chevrolet & Colorado & Pickup & Via VTRUX PU & Support \\
\hline G41-3153P & Dodge & Grd Caravan SE & Minivan & Honda Fit & Support \\
\hline
\end{tabular}




\begin{tabular}{|c|c|c|c|c|c|}
\hline G41-3159P & Dodge & Grd Caravan SE & Minivan & Honda Fit & Support \\
\hline G42-0587K & Chevrolet & Silverado & Pickup & Via VTRUX PU & Support \\
\hline G42-0590K & Chevrolet & Silverado & Pickup & Nissan eNV200 & Support \\
\hline G42-1232M & Ford & F150 & Pickup & Toyota Rav4 & Support \\
\hline G42-1281M & Ford & E-350 & Van - Pass & Via VTRUX Van & Support \\
\hline G43-3437B & Ford & E-350 & Van - Cargo & Nissan eNV200 & Support \\
\hline G61-0513K & Ford & Explorer & SUV & $\begin{array}{l}\text { Mitsubishi } \\
\text { Outlander }\end{array}$ & Support \\
\hline
\end{tabular}

\subsubsection{Commands Group Available Charge Time}

The Task 3 Vehicle Utilization report also identified the aggregated travel time summary for these monitored vehicles. The relevant section from that report is provided in Table 4.

Table 4. Pool vehicle travel time summary.

\begin{tabular}{|l|c|c|c|c|}
\hline \multicolumn{5}{|c|}{ Support Vehicles Travel Time Summary } \\
\hline & $\begin{array}{c}\text { Per Day } \\
\text { Average/Peak }\end{array}$ & $\begin{array}{c}\text { Per Outing } \\
\text { Average/Peak }\end{array}$ & $\begin{array}{c}\text { Per Trip } \\
\text { Average/Peak }\end{array}$ & Total \\
\hline Travel Time (Minutes) & $74.6 / 458.0$ & $38.0 / 1043.0$ & $455.5 / 264.0$ & 30,308 \\
\hline Idle Time (Minutes) & $13.3 / \mathrm{NA}$ & $5.4 / \mathrm{NA}$ & $1.7 / \mathrm{NA}$ & 5,964 \\
\hline
\end{tabular}

As shown above, the average daily usage is just over one hour per day. The peak daily usage was 7.6 hours. The peak outing time was higher because of overnight trips away from the home base. The distribution of daily travel times is shown in Figure 18. 47\% of daily travel time is of 20 minutes duration or less.

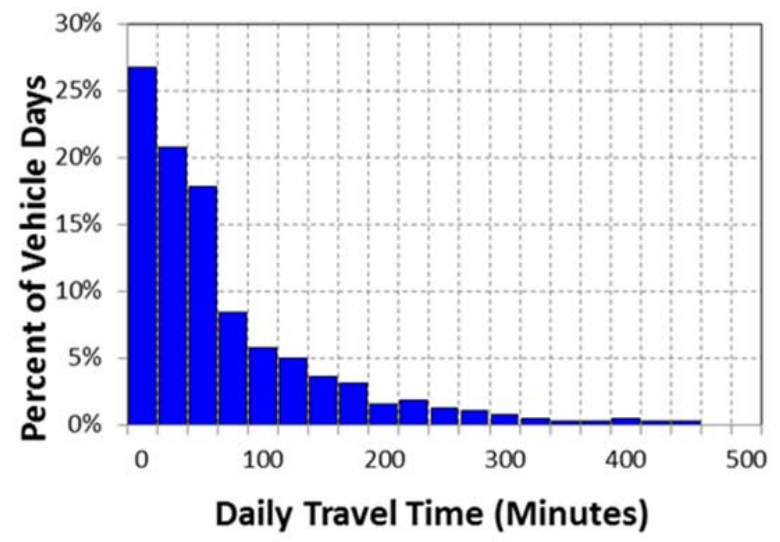

Figure 18. Support vehicle daily travel time

The Task 3 Vehicle Utilization report identified the Commands vehicles were operated on $45 \%$ of the study days at an average daily usage of 1.1 hours. In general, the vehicles are used on frequent days but average usage per day is quite low.

\subsubsection{Commands Group Electric Vehicle Supply Equipment Type Information}

As reported in the Task 3 Vehicle Utilization report, AC Level 2 overnight charging of BEVs is typical, whereas overnight charging of PHEVs can usually be accomplished with AC Level 1 charging. This would result in a typical recommendation to provide eleven AC Level 2 EVSEs for the BEVs and 
seven AC Level 1 EVSEs for the PHEVs. However, daily travel time and distance are typically so low that the time for recharge is not a concern.

\subsubsection{Commands Group Electric Vehicle Supply Equipment charging locations}

The home bases of the eighteen monitored vehicles reported by NASWI and recorded by instrument are shown in Table 5.

Table 5. Commands Group vehicle home base.

\begin{tabular}{|c|c|c|c|}
\hline Logger ID & Fleet Vehicle Id & Replacement PEV & Home Base \\
\hline 4 & G10-1138M & Rav4 & Bldg 385, Lexington St \\
\hline 7 & G10-1140M & eNV200 & Bldg 2593, Orion St \\
\hline 16 & G10-3576L & Rav4 & Bldg 993, Hospital, Franklin St \\
\hline 19 & G10-7547F & eNV200 & Bldg 386, Charles Porter Ave \\
\hline 38 & G41-1136K & Rav4 & Bldg 2547, Essex Rd \\
\hline 40 & G41-1139K & VTRUX PU & Bldg 410, Charles Porter Ave \\
\hline 41 & G41-1140K & eNV200 & Bldg 410, Charles Porter Ave \\
\hline 43 & G41-1155K & eNV200 & Bldg 993, Hospital, Franklin St \\
\hline 47 & G41-1351G & VTRUX PU & Bldg 2544, Prowler St \\
\hline 74 & G41-2911M & VTRUX PU & Bldg 975, Midway St \\
\hline 75 & G41-3153P & VTRUX PU & Bldg 385, Charles Porter Ave \\
\hline 76 & G41-3159P & eNV200 & Bldg 976, Princeton St \\
\hline 80 & G42-0587K & eNV200 & No Data (Bldg 410) \\
\hline 82 & G42-0590K & VTRUX PU & Bldg 385, Charles Porter Ave \\
\hline 90 & G42-1232M & eNV200 & Bldg 2737, Lexington St \\
\hline 91 & G42-1281M & Rav4 & Bldg 2737, Lexington St \\
\hline 100 & G43-3437B & Rav4 & Bldg 2642, Essex Rd \\
\hline 103 & G61-0513K & VTRUX Van & Bldg 2897, Ranger St \\
\hline
\end{tabular}

*Insufficient data was recorded to identify the home base for Vehicle G42-0587K. However, based upon its assignment to VQ-1, Building 410 on Charles Porter Ave is suggested.

Figure 19 maps the home base locations. 


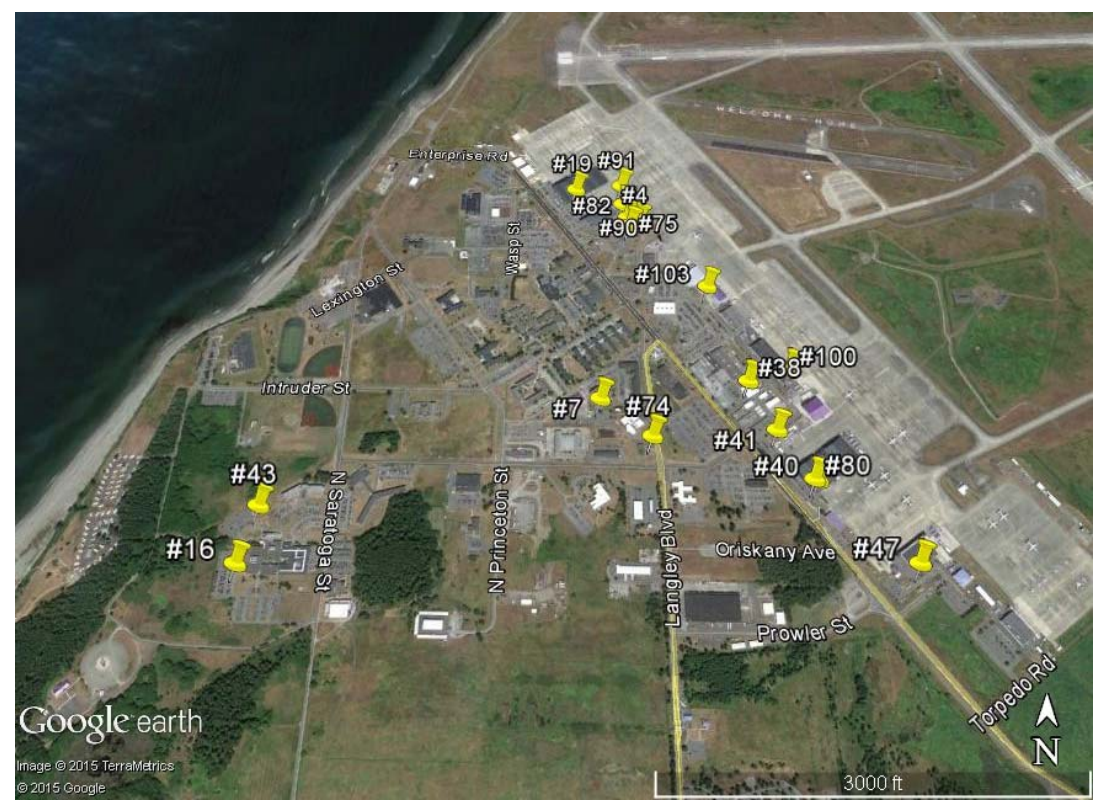

Figure 19. Commands Group home bases.

The vehicles for the Commands Group typically park overnight at their home base. At times PEVs benefit from additional charge opportunities if EVSE are located in areas where they frequently stop. Figure 20 shows locations where these vehicles frequently parked. The size of the symbol indicates the number of times parked in that location.

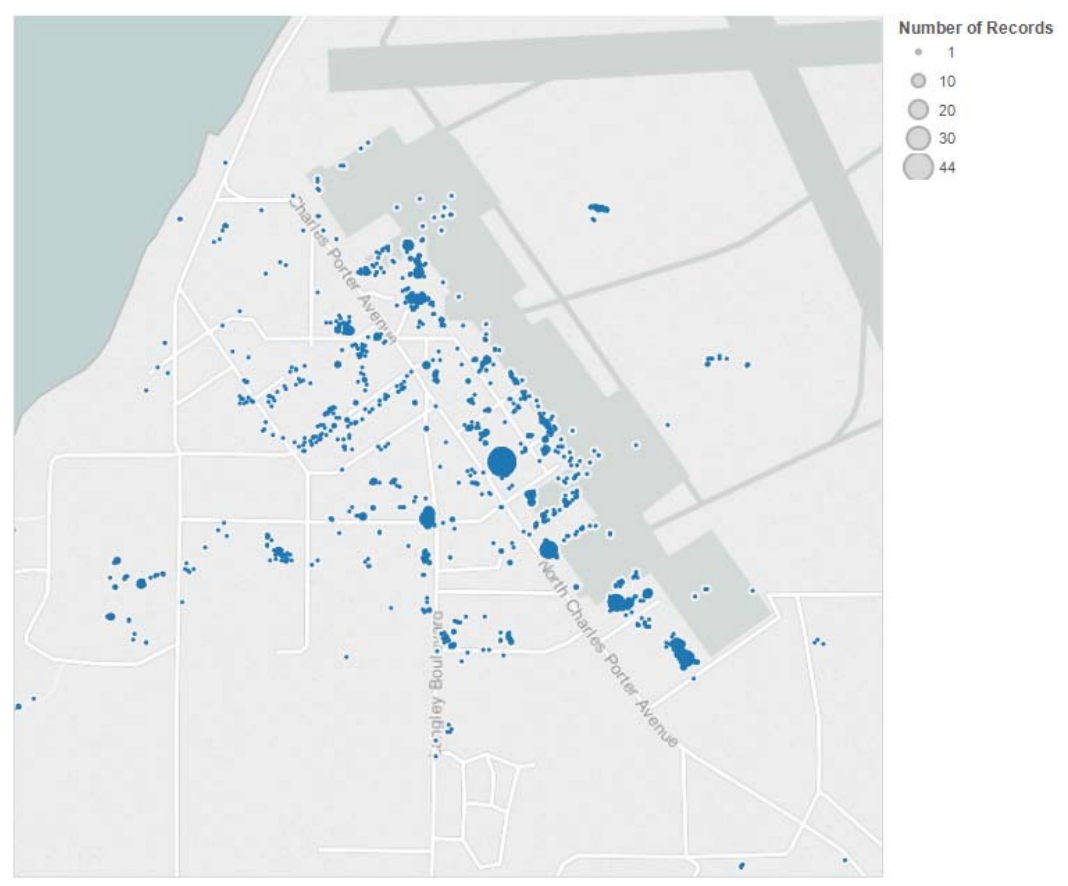

Figure 20. Commands vehicles frequent park locations.

While of interest, the number of times a vehicle parks in a location is of little value in determining where to place EVSE. Rather, the length of time a vehicle is parked at a particular location is of more value as some recharge may occur at the EVSE. 
Figure 21identifies the locations where Commands vehicles parked for more than 2 hours. The size of the symbol indicates the length of time parked capped at one week's duration.

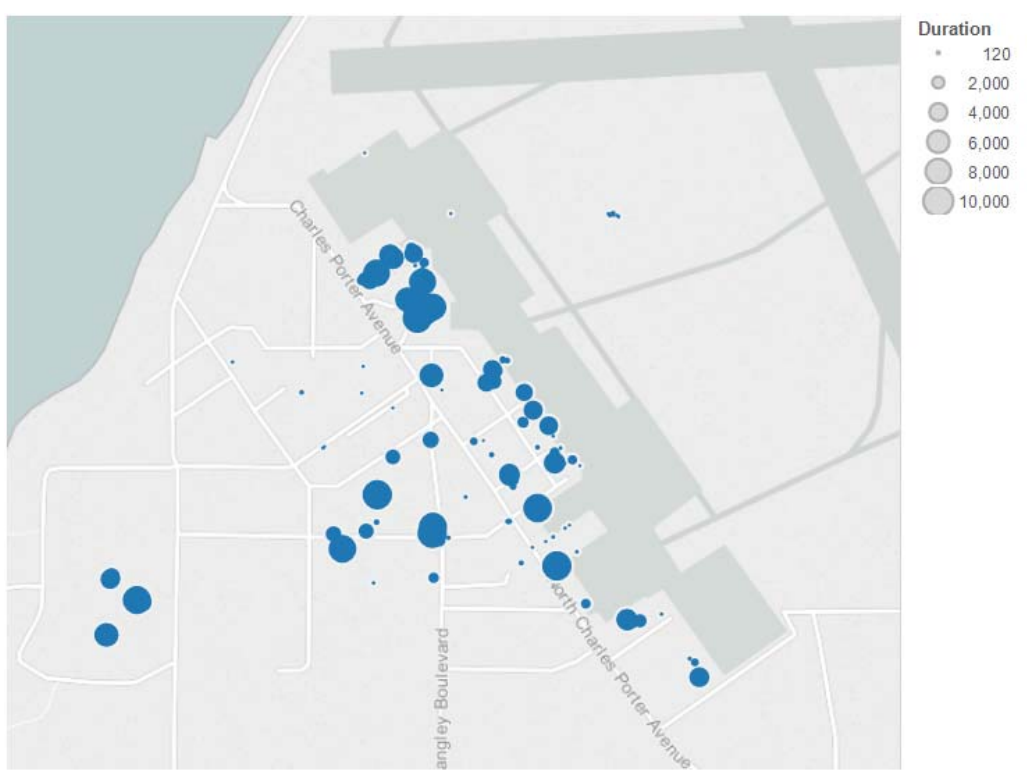

Figure 21. Commands Group vehicle stop locations by duration.

The locations identified in Figure 21 are essentially the home base locations. As noted previously, sufficient recharge time is available at the home base locations to reduce the desirability of adding EVSE stations elsewhere. Details of potential EVSE locations are shown in Section 5.

Figures 20 and 21 show that although the vehicles may have parked for significant time away from the home base, the number of times are insignificant compared to home-base parking.

\subsubsection{Commands Group Electric Vehicle Supply Equipment Observations}

Data show that the recommended PEVs should have sufficient time daily for recharging at the home base. The infrequent nature of the away-from-home base charging suggests that such would not be of much benefit. Thus, home base charging only is suggested. Most of the Commands vehicles are parked in unique locations.

Eleven BEVs and seven PHEV suggest that eleven AC Level 2 EVSEs and seven AC Level 1 EVSEs would be appropriate. Table 5 shows that in several cases, a single vehicle is assigned to a particular building and thus, a single EVSE will be required at that building. However, several buildings do have several vehicles assigned. Table 6 provides the identification of the number and type of EVSE for each building. Section 5 provides suggested locations for these EVSE.

It was suggested above that when installing EVSE, at least two of the type should be installed to reduce installation costs. However, because many of these sites have single vehicles assigned, the site may benefit from the installation of the single EVSE with all the preparations made for the second unit without actually installing it. This "stub-up" arrangement would allow the second unit to be installed later when the demand for that unit occurs.

Table 6. EVSE assignments by building.

\begin{tabular}{|c|c|c|c|c|c|}
\hline BIdg & $\begin{array}{c}\text { Vehicles } \\
\text { Assigned }\end{array}$ & $\begin{array}{c}\text { BEVs } \\
\text { Recommended }\end{array}$ & $\begin{array}{c}\text { PHEVs } \\
\text { Recommended }\end{array}$ & $\begin{array}{c}\text { AC L2 } \\
\text { EVSE }\end{array}$ & $\begin{array}{c}\text { AC L1 } \\
\text { EVSE }\end{array}$ \\
\hline 385 & 3 & 2 & 1 & 2 & 1 \\
\hline 386 & 1 & 1 & - & 1 & - \\
\hline
\end{tabular}




\begin{tabular}{|c|c|c|c|c|c|}
\hline 410 & 3 & 2 & 1 & 2 & 1 \\
\hline 975 & 1 & - & 1 & - & 1 \\
\hline 976 & 1 & 1 & - & 1 & - \\
\hline 993 & 2 & - & 2 & - & 2 \\
\hline 2544 & 1 & 1 & - & 1 & - \\
\hline 2547 & 1 & 1 & - & 1 & - \\
\hline 2593 & 1 & 1 & - & 1 & - \\
\hline 2642 & 1 & 1 & - & 1 & - \\
\hline 2737 & 2 & 1 & 1 & 1 & 1 \\
\hline 2897 & 1 & - & 1 & - & 1 \\
\hline Total & $\mathbf{1 8}$ & $\mathbf{1 1}$ & $\mathbf{7}$ & $\mathbf{1 1}$ & $\mathbf{7}$ \\
\hline
\end{tabular}

Building 385 also has Departments monitored vehicles assigned. Those vehicles are addressed in the next section.

The utilization of these Commands vehicles suggests that eleven AC Level 2 EVSE and seven AC Level 1 EVSE ports will be sufficient. Connecting the vehicles overnight should provide sufficient recharge time for all vehicles.

\subsubsection{Commands Group Fleet Summary}

This study provides observations for the vehicles monitored and for the entire non-tactical fleet of vehicles identified with the Commands Group.

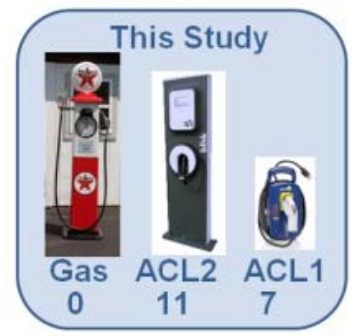

The fleet of support vehicles in this study included four sedans, six minivans, one SUV, one cargo van, one passenger van, and five pickup trucks. Intertek suggests that replacing these eighteen vehicles with eleven BEVs and seven PHEVs would meet current mission requirements and that eleven AC Level 2 and seven AC Level 1 EVSE ports should be sufficient for recharging.

Considering a full complement of 69 support vehicles in the Commands Group fleet, Intertek suggests a fleet of one conventional passenger van, 42 BEVs, and 27 PHEVs conservatively meets vehicle travel requirements. It is surmised that many of these vehicles will be assigned to the buildings identified above and that the PEVs can share the charging times at the EVSE. All Commands Group vehicles have a support mission and assuming that the full fleet operates in a manner similar to those monitored, it is likely that 28 AC Level 2 and 18 AC Level 1 EVSE could provide the necessary recharge capabilities with management attention to ensure all vehicles are rotated on the equipment and receive their recharge.

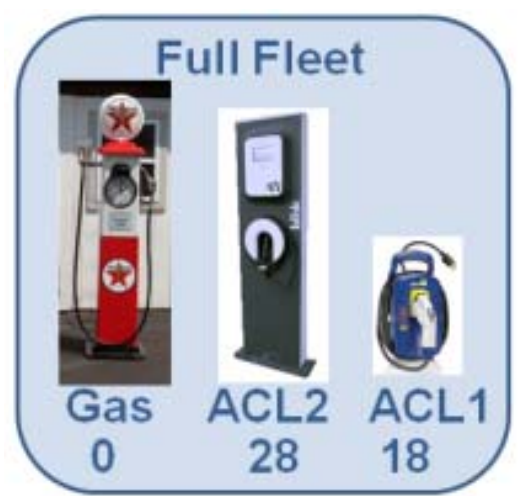

These suggestions be factored into further observations and suggestions related to the business case and schedule for any replacements for the Commands Group. Those observations will be addressed in Task 4 of this project.

\subsection{Analysis Results - Departments Group}

This section summarizes and aggregates the data collection for the Departments Group fleet. The details of each vehicle monitored are included in Appendix $\mathrm{C}$ of the Task 3 Vehicle Utilization report. 


\subsubsection{Departments Group recommended Plug-in Electric Vehicles for monitored vehicles}

The Task 3 Vehicle Utilization report recommended replacement of the monitored vehicles with those identified in Table 7. See that report for vehicle analysis. Seven vehicles had a pool mission, seven had enforcement mission, and twenty-eight were support vehicles.

Table 7. Departments Group monitored vehicle replacements

\begin{tabular}{|c|c|c|c|c|c|}
\hline \multicolumn{6}{|c|}{ Vehicle Index } \\
\hline $\begin{array}{c}\text { Fleet } \\
\text { Vehicle Id }\end{array}$ & Make & Model & EPA Class & $\begin{array}{c}\text { Replacement } \\
\text { PEV }\end{array}$ & Mission \\
\hline G10-0984N & Dodge & Avenger & Sedan - Compact & Focus & Support \\
\hline G10-1137M & Chevrolet & Malibu & Sedan - Midsize & Leaf & Support \\
\hline G10-1139M & Chevrolet & Malibu & Sedan - Midsize & Fusion & Support \\
\hline G10-1141M & Chevrolet & Malibu & Sedan - Midsize & Fusion & Support \\
\hline G10-2844L & Chevrolet & Malibu & Sedan - Midsize & Fusion & Pool \\
\hline G10-3590P & Chevrolet & Malibu & Sedan - Midsize & Leaf & Support \\
\hline G10-2848L & Chevrolet & Malibu & Sedan - Midsize & Fusion & Pool \\
\hline G10-2850L & Chevrolet & Malibu & Sedan - Midsize & Fusion & Pool \\
\hline G10-2968L & Chevrolet & Malibu & Sedan - Midsize & Fusion & Pool \\
\hline G10-5286H & Pontiac & G6 & Sedan - Compact & Volt & Support \\
\hline G42-1940N & Chevrolet & G1300 & Van - Pass & VTRUX Van & Pool \\
\hline G61-0245G & Ford & Escape & SUV & Outlander & Pool \\
\hline G11-0472L & Chevrolet & Impala & Sedan - Large & Fusion & Enforcement \\
\hline G11-1424L & Chevrolet & Impala & Sedan - Large & Fusion & Enforcement \\
\hline G11-2898K & Chevrolet & Impala & Sedan - Large & Fusion & Enforcement \\
\hline G41-1137K & Dodge & Grd Caravan SE & Minivan & Fit & Support \\
\hline G41-1142K & Dodge & Grd Caravan SE & Minivan & Outlander & Pool \\
\hline G41-1349G & Dodge & Dakota & Pickup & Rav4 & Support \\
\hline G41-1350G & Dodge & Dakota & Pickup & eNV200 & Support \\
\hline G41-1536L & Dodge & Grd Caravan Ex & Minivan & Fit & Support \\
\hline G41-1597L & Dodge & Dakota & Pickup & eNV200 & Support \\
\hline G41-1763H & Ford & Ranger & Pickup & Rav4 & Support \\
\hline G41-1765H & Ford & Ranger & Pickup & VTRUX PU & Support \\
\hline G41-1767H & Ford & Ranger & Pickup & eNV200 & Support \\
\hline G41-1768H & Ford & Ranger & Pickup & eNV200 & Support \\
\hline G41-4334M & Chevrolet & Colorado & Pickup & VTRUX PU & Support \\
\hline G42-0334G & Chevrolet & G1300 & Van - Pass & VTRUX Van & Pool \\
\hline G42-0335G & Chevrolet & G1300 & Van - Pass & VTRUX Van & Support \\
\hline G42-0656K & Chevrolet & Silverado & Pickup & eNV200 & Support \\
\hline G42-0766L & Chevrolet & $\mathrm{C} 1500$ & Pickup & VTRUX PU & Support \\
\hline G42-0881H & Ford & F150 & Pickup & eNV200 & Pool \\
\hline G42-1222M & Chevrolet & G1300 Express & Van - Pass & VTRUX Van & Support \\
\hline G42-1223M & Chevrolet & G1300 Express & Van - Pass & eNV200 & Support \\
\hline G42-2035L & Chevrolet & Tahoe & SUV & Fit & Enforcement \\
\hline
\end{tabular}




\begin{tabular}{|c|c|c|c|c|c|}
\hline G43-0895K & Ford & E-350 & Van - Pass & eNV200 & Support \\
\hline G43-0896K & Ford & E-350 & Van - Pass & eNV200 & Support \\
\hline G43-0936K & Chevrolet & C2500HD & Van - Cargo & eNV200 & Enforcement \\
\hline G43-2287M & Chevrolet & G2300 & Van - Pass & eNV200 & Support \\
\hline G43-2288M & Chevrolet & G2300 Express & Van - Pass & eNV200 & Support \\
\hline G62-0203H & Ford & F150 & Pickup & Rav4 & Support \\
\hline G62-0871K & Ford & Expedition & SUV & Outlander & Enforcement \\
\hline G62-2420P & Ford & FI50 SSV & Pickup & VTRUX Van & Enforcement \\
\hline
\end{tabular}

\subsubsection{Departments Group available charge time}

The Task 3 Vehicle Utilization report also identified the aggregated travel time summary for these monitored vehicles. The relevant sections from that report for pool, support, and enforcement vehicles are provided in Tables 8, 9, and 10 .

Table 8. Pool vehicles travel summary.

\begin{tabular}{|l|c|c|c|c|}
\hline \multicolumn{5}{|c|}{ Pool Vehicles Travel Summary } \\
\hline & $\begin{array}{c}\text { Per Day } \\
\text { Average/Peak }\end{array}$ & $\begin{array}{c}\text { Per Outing } \\
\text { Average/Peak }\end{array}$ & $\begin{array}{c}\text { Per Trip } \\
\text { Average/Peak }\end{array}$ & Total \\
\hline Travel Time (Minutes) & $125.4 / 486.0$ & $143.7 / 1202.0$ & $26.2 / 237.0$ & 14,802 \\
\hline Idle Time (Minutes) & $10.6 / \mathrm{NA}$ & $12.2 / \mathrm{NA}$ & $2.2 / \mathrm{NA}$ & 1,255 \\
\hline
\end{tabular}

The average daily usage for the pool vehicles is about two hours per day. The peak daily usage was about 8 hours. The peak outing time was higher due to overnight trips away from the home base.

Table 9. Support vehicles travel summary.

\begin{tabular}{|l|c|c|c|c|}
\hline \multicolumn{5}{|c|}{ Support Vehicles Travel Summary } \\
\hline & $\begin{array}{c}\text { Per Day } \\
\text { Average/Peak }\end{array}$ & $\begin{array}{c}\text { Per Outing } \\
\text { Average/Peak }\end{array}$ & $\begin{array}{c}\text { Per Trip } \\
\text { Average/Peak }\end{array}$ & Total \\
\hline Travel Time (Minutes) & $49.0 / 338.0$ & $19.4 / 338.0$ & $9.2 / 192.0$ & 30,971 \\
\hline Idle Time (Minutes) & $14.9 /$ NA & $5.9 /$ NA & $2.8 /$ NA & 9,426 \\
\hline
\end{tabular}

The average daily usage for support vehicles is less than one hour per day. The longest daily travel was under six hours.

Table 10. Enforcement vehicle travel summary

\begin{tabular}{|l|c|c|c|c|}
\hline \multicolumn{5}{|c|}{ Enforcement Vehicle Travel Summary } \\
\hline & $\begin{array}{c}\text { Per Day } \\
\text { Average/Peak }\end{array}$ & $\begin{array}{c}\text { Per Outing } \\
\text { Average/Peak }\end{array}$ & $\begin{array}{c}\text { Per Trip } \\
\text { Average/Peak }\end{array}$ & Total \\
\hline Travel Time (Minutes) & $313.5 / 1232.0$ & $86.0 / 1072.0$ & $25.5 / 555.0$ & 79,315 \\
\hline Idle Time (Minutes) & $124.8 /$ NA & $34.2 /$ NA & $10.2 /$ NA & 31,570 \\
\hline
\end{tabular}

The average daily usage for enforcement vehicles is about five hours per day. The longest daily usage was over 20 hours. 
The distribution of daily travel times are shown in Figure 22.
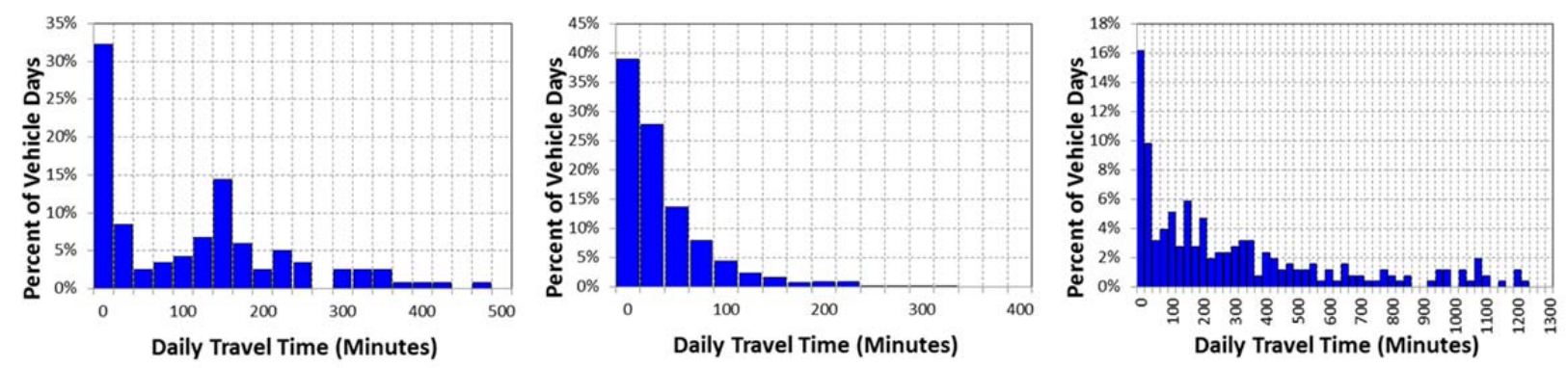

Figure 22. Pool, support, and enforcement vehicle daily travel minutes (all vehicles).

The Task 3 Vehicle Utilization report identified the Departments vehicles were operated on $50 \%$ of the study days at an average daily usage of 1.8 hours. In general, the vehicles are used on frequent days but average usage per day is quite low.

\subsubsection{Departments Group Electric Vehicle Supply Equipment Type Recommendation}

As reported in the Task 3 Vehicle Utilization report, AC Level 2 overnight charging of BEVs is typical, whereas overnight charging of PHEVs can usually be accomplished with AC Level 1 charging. This would result in a typical recommendation to provide 21AC Level 2 EVSE for the BEVs and 21 AC Level 1 EVSE for the PHEVs.

\subsubsection{Departments Group Electric Vehicle Supply Equipment Charging Locations}

The home bases of the 42 Department monitored vehicles reported by NASWI and recorded by instrument are shown in Table 11.

Table 11. Departments Group vehicle home base.

\begin{tabular}{|c|c|c|}
\hline Fleet Vehicle Id & Replacement PEV & Home Base \\
\hline G10-0984N & Focus & Bldg 382, Charles Porter Ave \\
\hline G10-1137M & Leaf & Bldg 243, Midway St \\
\hline G10-1139M & Fusion & Bldg 103, Eisenhower St \\
\hline G10-1141M & Fusion & Bldg 103, Eisenhower St \\
\hline G10-2844L & Fusion & Bldg 124, Midway St \\
\hline G10-3590P & Leaf & Bldg 243, Midway St. \\
\hline G10-2848L & Fusion & Bldg 124, Midway St \\
\hline G10-2850L & Fusion & Bldg 124, Midway St \\
\hline G10-2968L & Fusion & Bldg 124, Midway St \\
\hline G10-5286H & Volt & Bldg 960, Chapel, Intruder St \\
\hline G42-1940N & VTRUX Van & Bldg 124, Midway St \\
\hline G61-0245G & Outlander & Bldg 386, Charles Porter Ave \\
\hline G11-0472L & Fusion & Bldg 994, Charles Porter Ave \\
\hline G11-1424L & Fusion & Bldg 994, Charles Porter Ave \\
\hline G11-2898K & Fusion & Bldg 994, Charles Porter Ave \\
\hline G41-1137K & Fit & Bldg 2556, Lexington St \\
\hline G41-1142K & Outlander & Bldg 385, Lexington St \\
\hline
\end{tabular}




\begin{tabular}{|c|c|c|}
\hline G41-1349G & Rav4 & Bldg 378, Intruder St \\
\hline G41-1350G & eNV200 & Bldg 385, Lexington St \\
\hline G41-1536L & Fit & Bldg 103, Eisenhower St \\
\hline G41-1597L & eNV200 & Bldg 103, Eisenhower St \\
\hline G41-1763H & Rav4 & Bldg 2734, Lexington St \\
\hline G41-1765H & VTRUX PU & No Data (Bldg 385) \\
\hline G41-1767H & eNV200 & Bldg 103, Eisenhower St \\
\hline G41-1768H & eNV200 & No Data (Bldg 385) \\
\hline G41-4334M & VTRUX PU & Bldg 2699, Ranger St \\
\hline G42-0334G & VTRUX Van & Bldg 124, Midway St \\
\hline G42-0335G & VTRUX Van & Bldg 2704, Prowler St \\
\hline G42-0656K & eNV200 & Bldg 103, Eisenhower St \\
\hline G42-0766L & VTRUX PU & Bldg 2555, Langley Blvd \\
\hline G42-0881H & eNV200 & Bldg 124, Midway St \\
\hline G42-1222M & VTRUX Van & Bldg 385, Lexington St \\
\hline G42-1223M & eNV200 & Bldg 2734, Lexington St \\
\hline G42-2035L & Fit & Bldg 994, Charles Porter Ave \\
\hline G43-0895K & eNV200 & Bldg 2734, Lexington St \\
\hline G43-0896K & eNV200 & Bldg 2734, Lexington St \\
\hline G43-0936K & eNV200 & Bldg 2815, Langley Blvd \\
\hline G43-2287M & eNV200 & Bldg 2737, Lexington St \\
\hline G43-2288M & eNV200 & Bldg 385, Lexington St \\
\hline G62-0203H & Rav4 & No Data (Bldg 103) \\
\hline G62-0871K & Outlander & Bldg 994, Charles Porter Ave \\
\hline G62-2420P & VTRUX Van & Charles Porter Ave \\
\hline
\end{tabular}

Insufficient data was collected to identify the home bases for Vehicles G41-1765 H, G41-1768H, and G62-0203H. The above locations are assumed based on similar group assignments within Departments.

Figure 23 illustrates these home base locations.

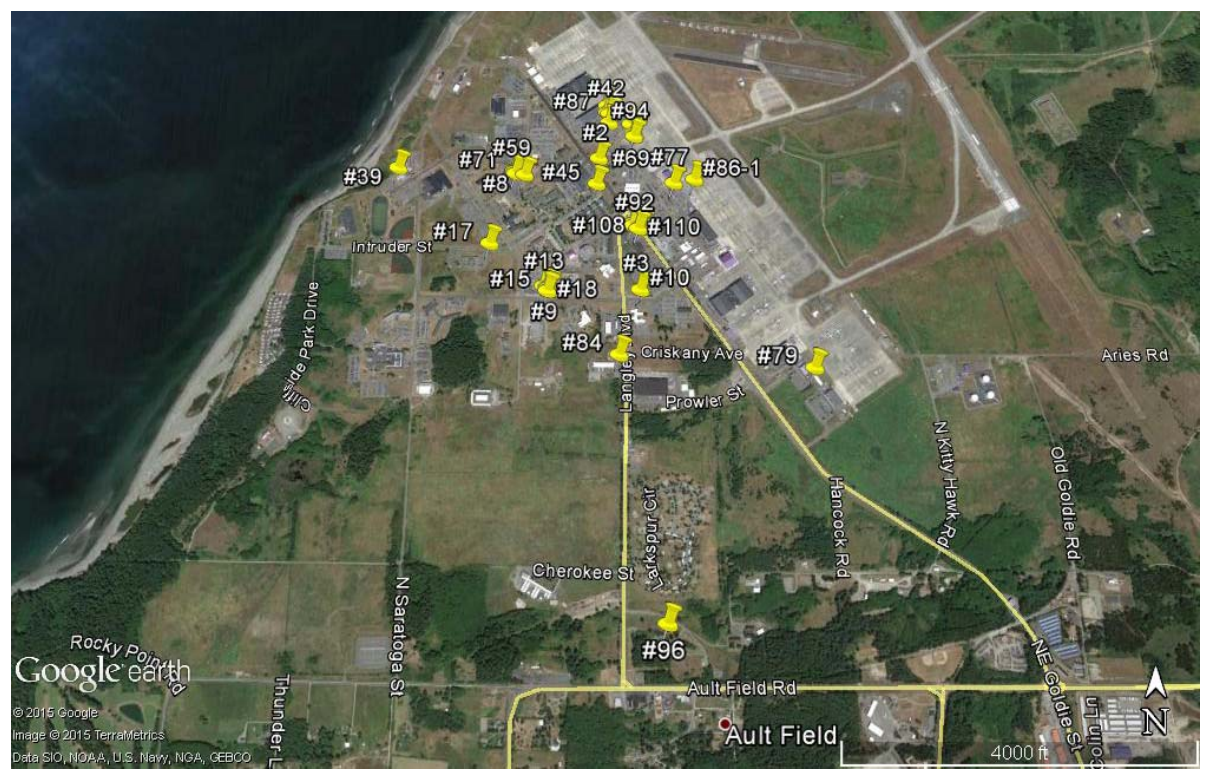


Figure 23. Departments Group home locations.

The vehicles for the Departments Group typically park overnight at their home base. At times PEVs benefit from additional charge opportunities if EVSE are located in areas where they frequently stop.

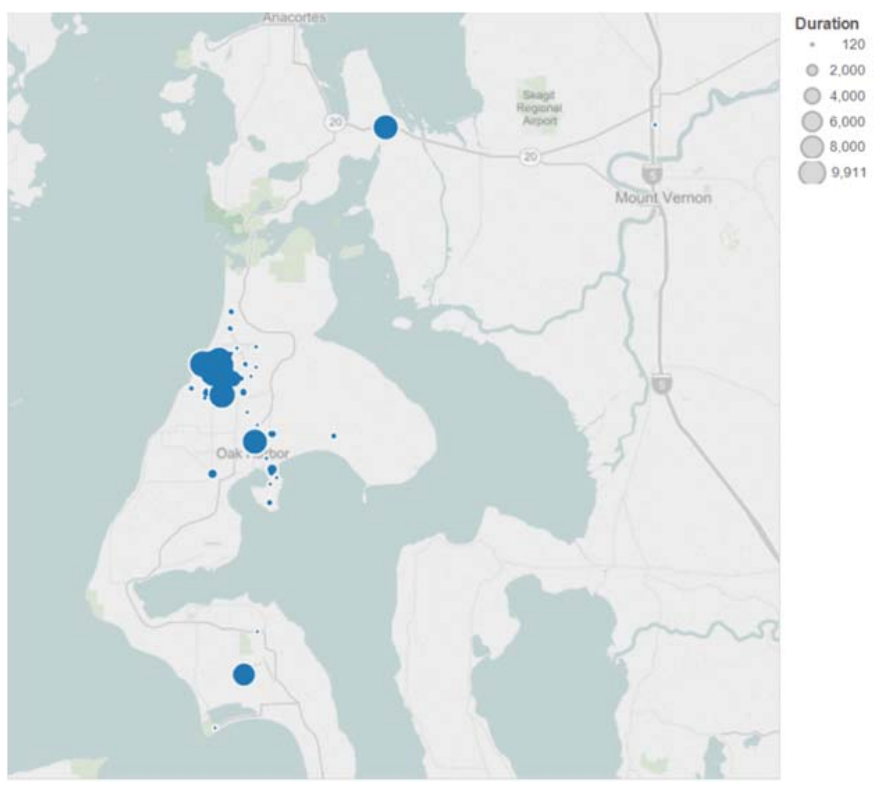

Figure 24. Departments Group vehicle stop locations by duration.

Figure 24 shows locations where these vehicles parked for more than 2 hours. The size of the symbol indicates the length of time parked at that location capped at one week's duration.

Figure 25 shows locations where these vehicles parked on base for more than 2 hours. Again, the time duration is capped at one week. 


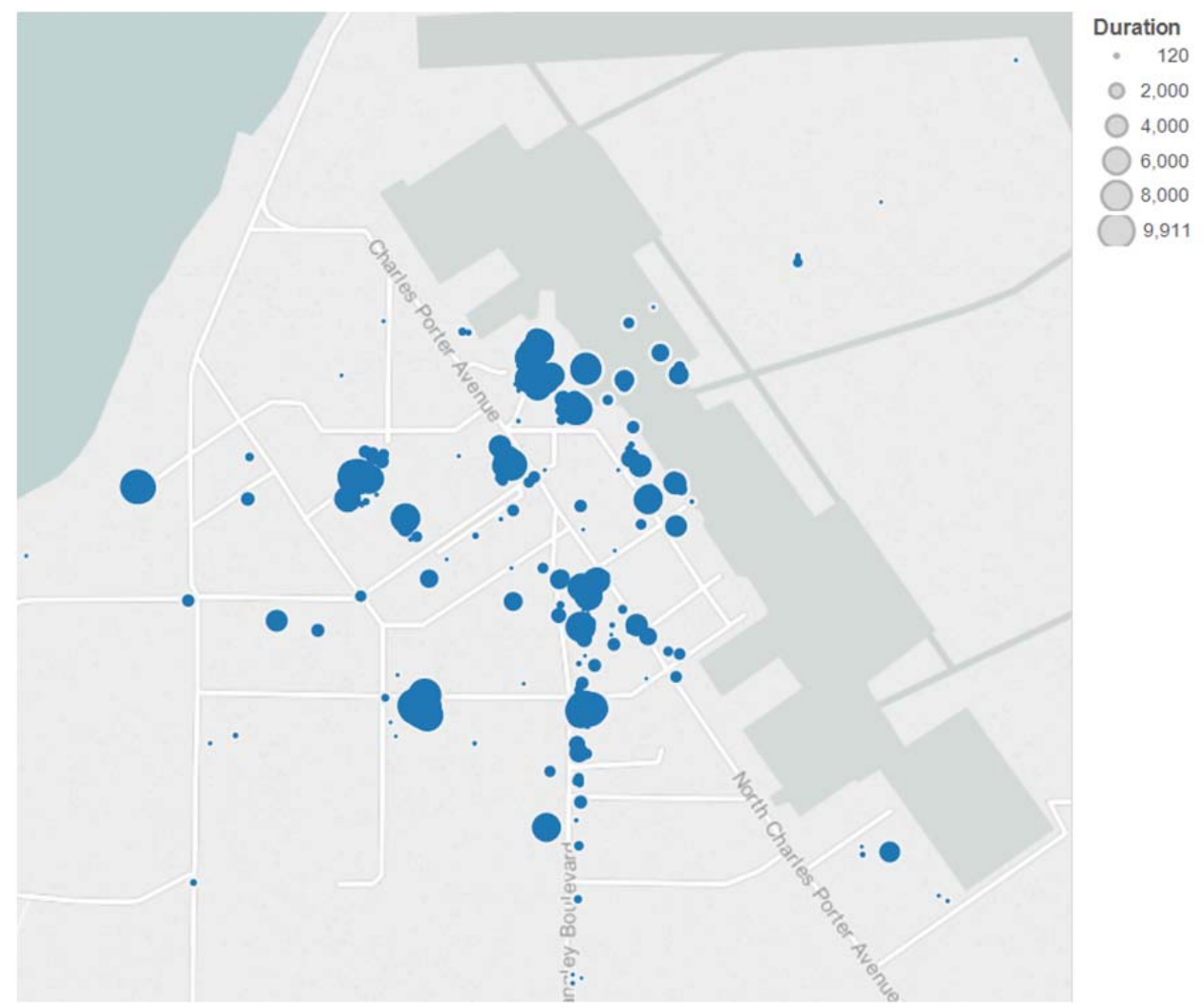

Figure 25. Departments Group vehicle stop locations on base greater than two hours.

Significant stops greater than two hours occur primarily at the vehicle's home base.

\subsubsection{Departments Group Electric Vehicle Supply Equipment observation}

Data show that the recommended PEVs should have sufficient time daily for recharging at the home base. The infrequent nature of the away-from-home base charging suggests that such would not be of much benefit. Thus, home base charging only is suggested. Most of the Departments vehicles are parked in unique locations.

Twenty-one BEVs and twenty-one PHEV suggest that 21 AC Level 2 and 21 AC Level 1 EVSE would be appropriate. Table 11 shows that in several cases, a single vehicle is assigned to a particular building and thus, a single EVSE will be required at that building. However, several buildings do have several vehicles assigned. Table 12 provides the identification of the number and type of EVSE for each building. Section 5 provides suggested locations for these EVSE.

It was suggested above that when installing EVSE, at least two of the type should be installed to reduce installation costs. However, because many of these sites have single vehicles assigned, the site may benefit from the installation of the single EVSE with all the preparations made for the second unit without actually installing it. This "stub-up" arrangement would allow the second unit to be installed later when the demand for that unit occurs.

Table 12. EVSE assignments by building.

\begin{tabular}{|c|c|c|c|c|c|}
\hline Bldg & $\begin{array}{c}\text { Vehicles } \\
\text { Assigned }\end{array}$ & $\begin{array}{c}\text { BEVs } \\
\text { Recommended }\end{array}$ & $\begin{array}{c}\text { PHEVs } \\
\text { Recommended }\end{array}$ & $\begin{array}{c}\text { AC L2 } \\
\text { EVSE }\end{array}$ & $\begin{array}{c}\text { AC L1 } \\
\text { EVSE }\end{array}$ \\
\hline 103 & 7 & 5 & 2 & 3 & 2 \\
\hline 124 & 8 & 1 & 7 & 1 & 4 \\
\hline 243 & 2 & 2 & - & 2 & - \\
\hline
\end{tabular}




\begin{tabular}{|c|c|c|c|c|c|}
\hline 378 & 1 & 1 & - & 1 & - \\
\hline 382 & 1 & 1 & - & 1 & - \\
\hline 385 & 6 & 3 & 3 & 2 & 2 \\
\hline 960 & 1 & - & 1 & - & 1 \\
\hline 994 & 6 & 1 & 5 & 1 & 3 \\
\hline 2555 & 1 & - & 1 & - & 1 \\
\hline 2556 & 1 & 1 & - & 1 & - \\
\hline 2699 & 1 & - & 1 & - & 1 \\
\hline 2704 & 1 & - & 1 & - & 1 \\
\hline 2734 & 4 & 4 & - & 4 & - \\
\hline 2737 & 1 & 1 & - & 1 & - \\
\hline 2815 & 1 & 1 & - & 1 & - \\
\hline Total & $\mathbf{4 2}$ & $\mathbf{2 1}$ & $\mathbf{2 1}$ & $\mathbf{1 8}$ & $\mathbf{1 5}$ \\
\hline
\end{tabular}

Building 385 also has Commands monitored vehicles assigned. Those vehicles were addressed in the previous section.

The utilization of these Departments vehicles suggests that 18 AC Level 2 EVSE and 15 AC Level 1 EVSE ports will be sufficient. Because the numbers of EVSE are less than the number of PEVs, management attention will be required to rotate PEVs on the appropriate EVSE. If management attention is not available, each PEV should be assigned a specific EVSE.

\subsubsection{Departments Group Summary}

This study provides observations for the vehicles monitored and for the entire non-tactical fleet of vehicles identified with the Departments Group.

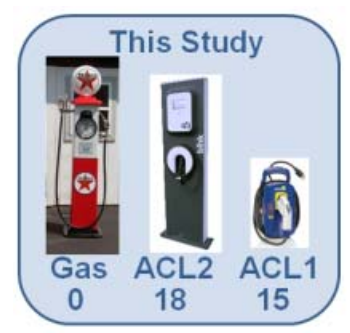

The vehicles monitored in this study included fifteen sedans, three minivans, two SUVS, one cargo van, eight passenger vans, and thirteen pickup trucks. Intertek suggests that replacing the vehicles with 21 BEV and 21 PHEVs would meet current mission requirements. Further, 18 AC Level 2 and 15 AC Level 1 EVSE ports should be sufficient for recharging.

Considering a full complement of 106 vehicles in the Departments Group fleet, Intertek suggests a fleet consisting of $\mathbf{5 0}$

BEVs and 56 PHEVs should meet mission objectives. Because the utilization of these vehicles is low, Intertek suggests 42 AC Level 2 and 40 AC Level 1 EVSE should meet recharging requirements.

Some management attention will be required to insure that the vehicles are effectively rotated on the AC Level 2 EVSE for charging and dispatched based upon the battery state of charge. It is likely that such attention is already employed when assigning pool vehicles. Connecting the vehicles overnight should provide

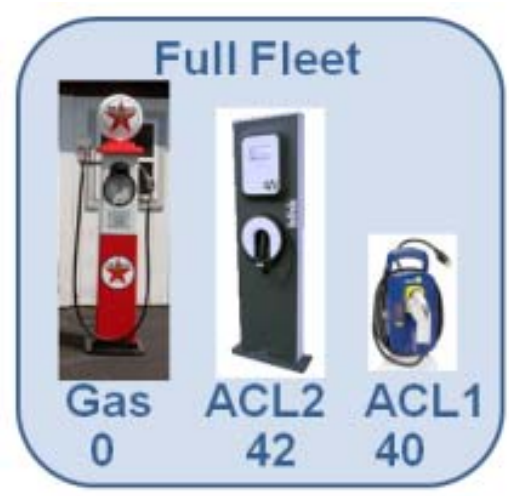
sufficient recharge time for all vehicles.

These suggestions be factored into further observations and suggestions related to the business case and schedule for any replacements for the Departments Group. Those observations will be addressed in Task 4 of this project. 


\subsection{Analysis Results - All Monitored Fleet}

This section summarizes and aggregates the data collection for the aggregated fleet of monitored vehicles. The details of each vehicle monitored are included in Appendix B and C of the Task 3 Vehicle Utilization report.

\subsubsection{Aggregated Fleet available charge time}

The aggregated travel time summary for all monitored vehicles is provided in Table 13.

Table 13. All vehicle travel summary

\begin{tabular}{|l|c|c|c|c|}
\hline \multicolumn{5}{|c|}{ Aggregated Vehicles Travel Summary } \\
\hline & $\begin{array}{c}\text { Per Day } \\
\text { Average/Peak }\end{array}$ & $\begin{array}{c}\text { Per Outing } \\
\text { Average/Peak }\end{array}$ & $\begin{array}{c}\text { Per Trip } \\
\text { Average/Peak }\end{array}$ & Total \\
\hline Travel Time (Minutes) & $112.2 / 1,232.0$ & $38.8 / 1,202.0$ & $15.5 / 14,367.0$ & 155,396 \\
\hline Idle Time (Minutes) & $34.8 /$ NA & $12.0 /$ NA & $4.8 /$ NA & 48,215 \\
\hline
\end{tabular}

The average daily usage for all vehicles is just under two hours per day. The longest daily travel was an enforcement vehicle usage of about 20.5 hours.

The frequented stop locations and durations are identified in the previous sections. There do not appear to be many locations other than home bases where the vehicles park frequently for long durations. Other than Building 385, there appear to be no locations for simultaneous home bases for both Commands and Departments vehicles. In addition, a review of the base map does not suggest other locations for EVSE installed for official business.

\subsubsection{Aggregated Fleet Summary}

This study provides observations for the vehicles monitored and for the entire non-tactical fleet of vehicles at NASWI.

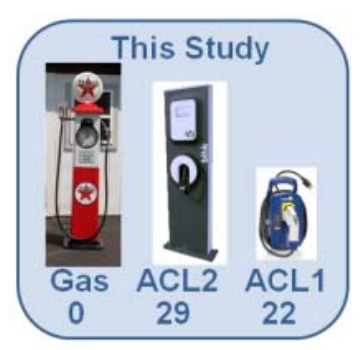

The vehicles monitored in this study included 60 internal combustion engine vehicles of types noted above. Intertek suggests that replacing the vehicles with 32 BEV and 28 PHEVs would meet current mission requirements. Further, 29 AC Level 2 and 22 AC Level 1 EVSE ports should be sufficient for recharging.

Considering a full complement of 175 vehicles in the Aggregated Fleet, Intertek suggests a fleet consisting of 92 BEVs and 83

PHEVs should meet mission objectives. Because the utilization of these vehicles is low, Intertek suggests 70 AC Level 2 and 58 AC Level 1 EVSE should meet recharging requirements.

Some management attention will be required to insure that the vehicles are effectively rotated on the AC Level 2 EVSE for charging and dispatched based upon the battery state of charge. It is likely that

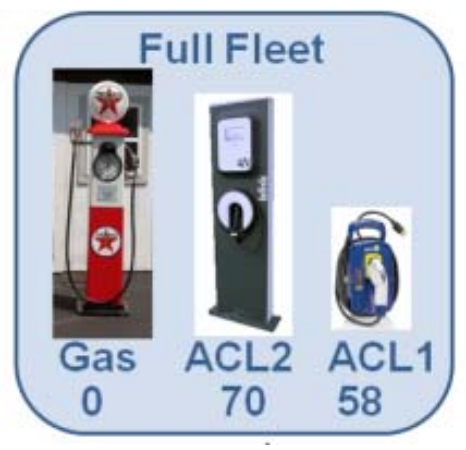
such attention is already employed when assigning pool vehicles. Connecting the vehicles overnight should provide sufficient recharge time for all vehicles.

These suggestions be factored into further observations and suggestions related to the business case and schedule for any replacements for the Aggregated Fleet. Those observations will be addressed in Task 4 of this project. 


\subsection{Public Charging Electric Vehicle Supply Equipment Locations}

The EVSE recommended in the previous sections are intended for fleet operations. Should NASWI be interested in providing charging stations to support charging of POVs, specific destination locations should be considered. Some of the points that attract the drivers of POVs are identified on the Base Map These include the recreation building, post office, theater, golf course club house, clubs, barracks, Navy Exchange/gas station, chapel, hospital, etc. Base access would be required to access these locations. Some of these locations are sites recommended for fleet EVSE but these would generally not be available for POVs.

NASWI has no direct access from an interstate. In some cases, this can be advantageous in providing DC fast charging locations for the public from which the base vehicles can benefit as well.

\section{NAVAL AIR STATION WHIDBEY ISLAND ELECTRIC VEHICLE SUPPLY EQUIPMENT LOCATIONS DETAILS}

\subsection{Background}

Section 3.3 provides guidance in site selection and installation of EVSE. The availability of electrical power near the desired EVSE location is the most important factor affecting installation costs. Locations nearer the electrical supply will result in shorter conduit and conductor runs to minimize costs. Locations near landscaped areas reduce costs by reducing the amount of asphalt or concrete cuts to install the conduit. At the same time, the location for the fleet vehicles should not be in the most ideal of parking locations for the facility if they are to be restricted for PEV charging only since the EVSE are not likely to be available to POVs and other non-fleet vehicles.

Section 5.2 provides some suggested locations for the majority of the monitored fleet vehicle EVSE. In most cases, parking areas near the buildings will produce the least distance from the power center. However, for some buildings that will be destinations for POVs and business other than the fleet vehicles, site selection may not be the closest building approach.

\subsection{Naval Air Station Whidbey Island Electric Vehicle Supply Equipment Locations}

NASWI provided the NAS Whidbey Island Ault Field - Base Map for use with this study. It identifies the major substations related to the electrical distribution. This is insufficient for detailed EVSE planning, as the EVSE location is specific to home base buildings. Detailed electrical drawings may be classified and are not available for this report. Potential EVSE locations identified are based upon apparent distances from buildings, available information on electrical sources, and ease of installation. Google Earth $^{24}$ provides the pictorial maps.

\subsubsection{Commands and EVSE Locations}

Table 5 identifies the home base locations for the Command Group monitored vehicles.

Figure 26 identifies a potential location near Building 385. Several vehicles are home-based near this building: Vehicles G10-1138M, G41-3153P, and G42-0590K assigned to Commands as well as many Department vehicles noted in the next section.

\footnotetext{
${ }^{24}$ http://www.google.com/earth/ [accessed March 7, 2015]
} 


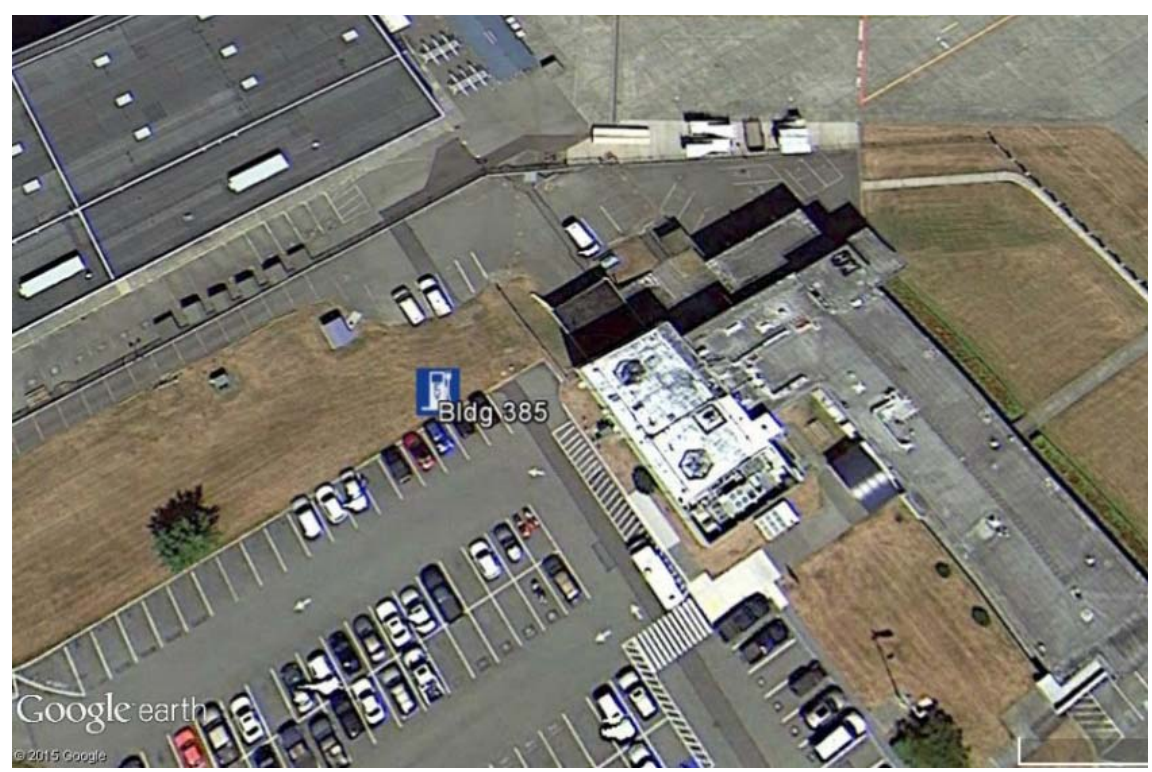

Figure 26. EVSE potential location near Bldg 385 for numerous vehicles.

Figure 27 identifies a potential location near Building 410. Several vehicles are home-based near this building: Vehicles G41-1139K, G41-1140K, and G42-0587K assigned to Commands.

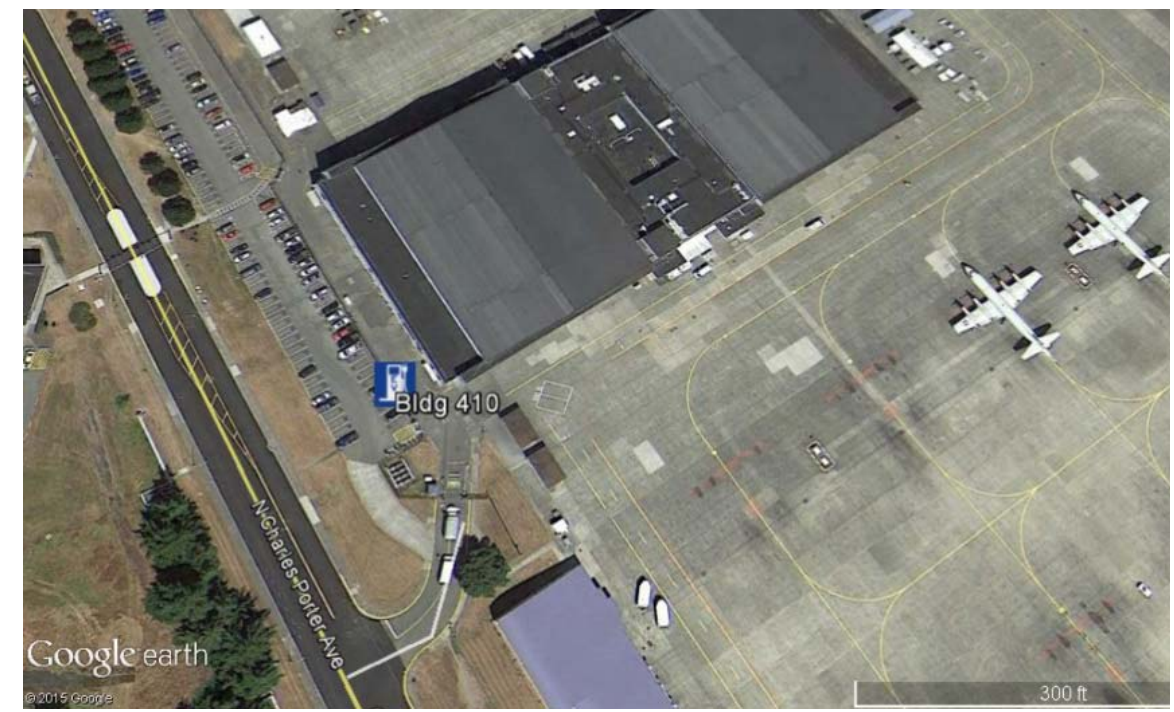

Figure 27. EVSE potential location near Bldg 410 for numerous vehicles. 


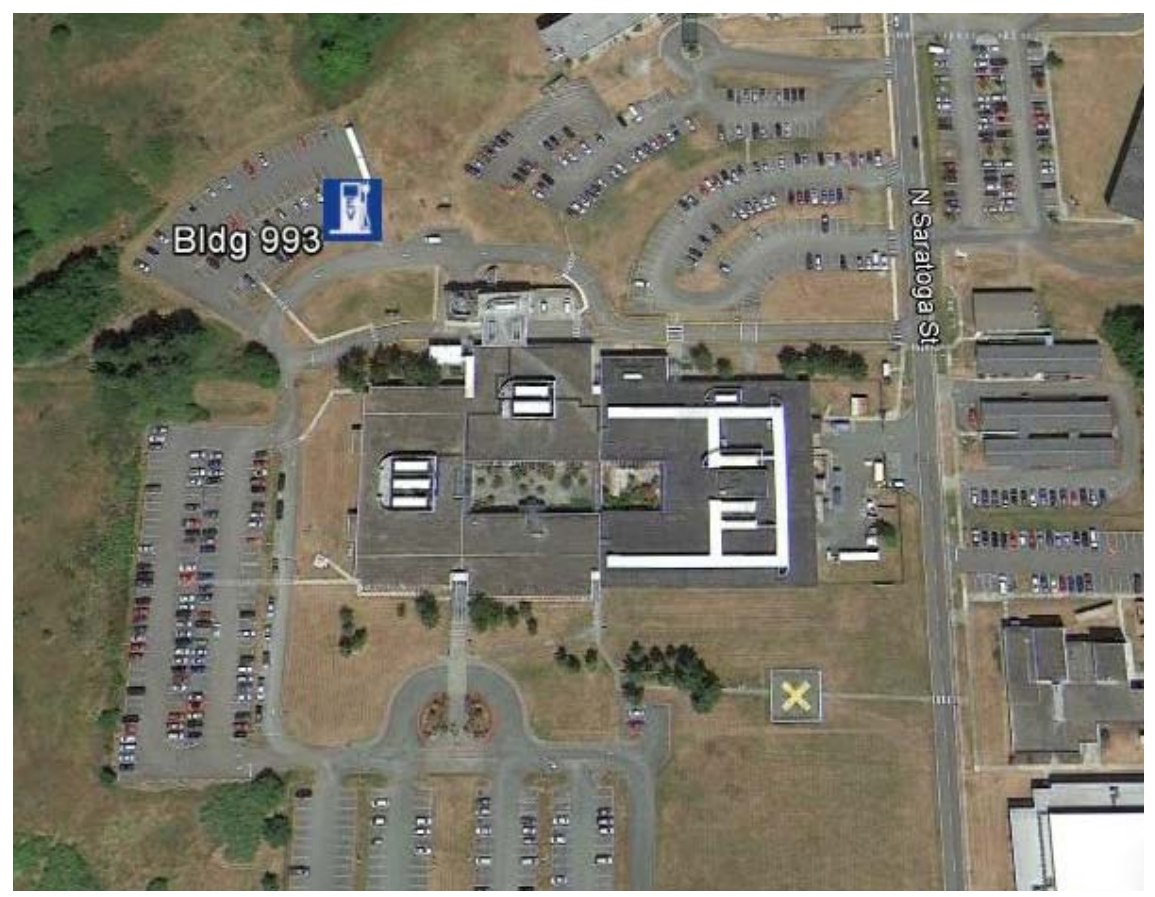

Figure 28 identifies a potential location near Building 993. Vehicles G10-3576L and G41-1155K assigned to Commands are home based near this building.

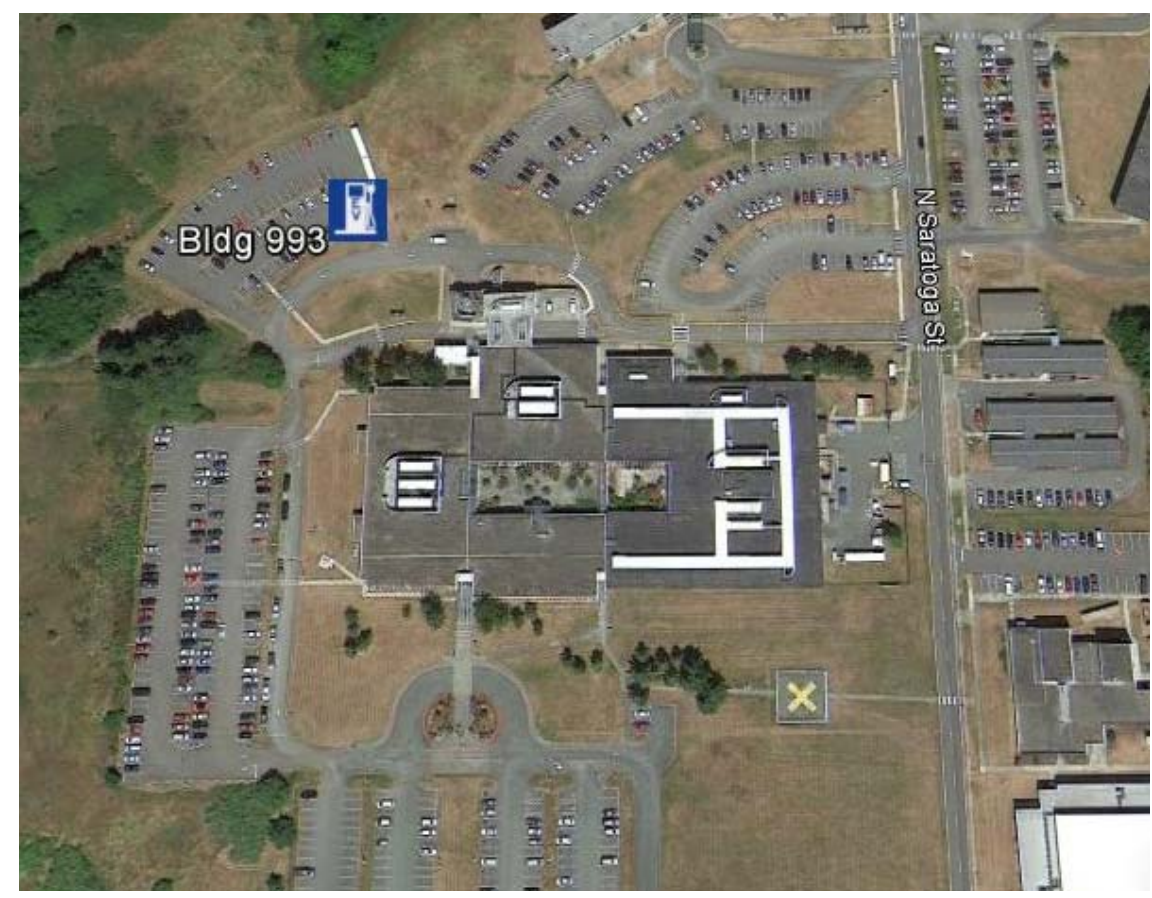

Figure 28. EVSE potential location near Bldg 993

Figure 29 identifies a potential location near Building 2737. Vehicles G42-1232M and G42-1281M assigned to Commands are home based near this building. 


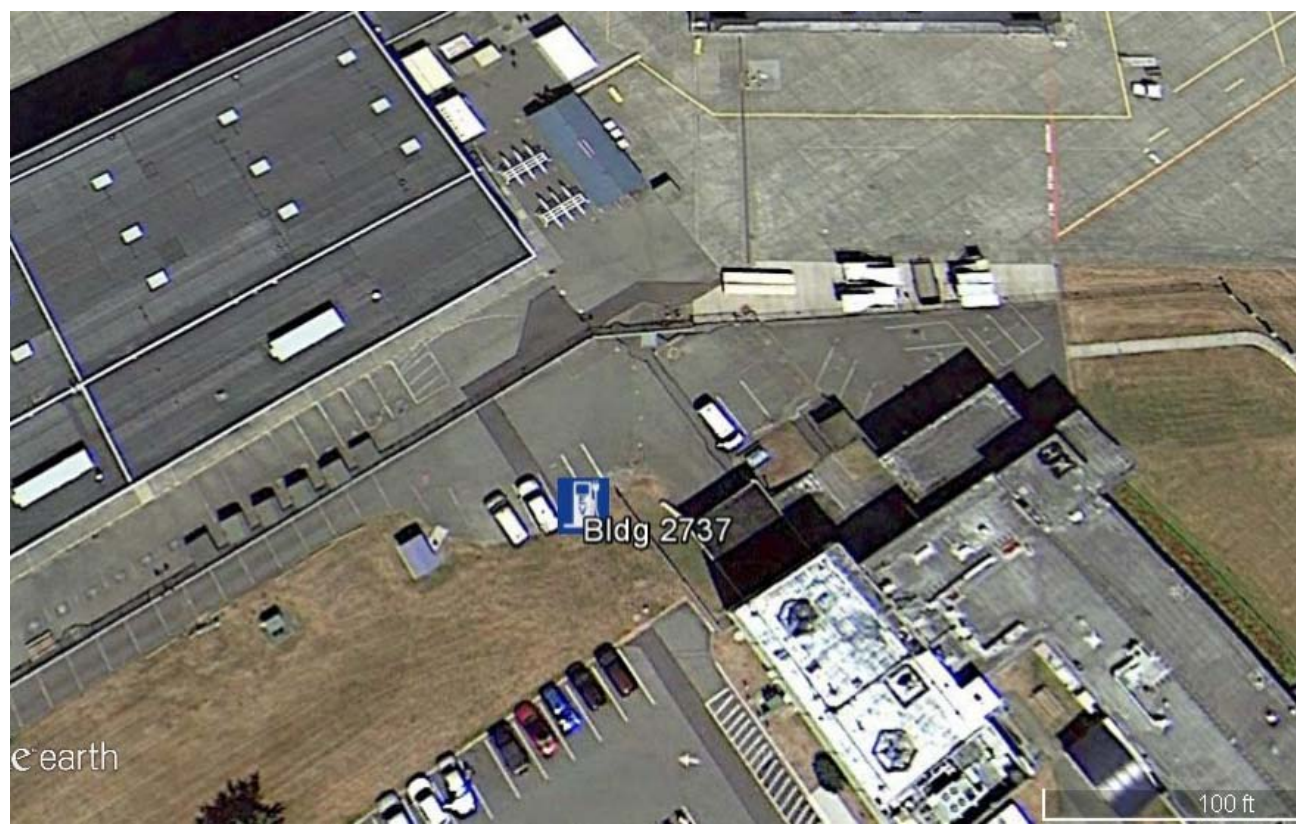

Figure 29. EVSE potential location near Bldg 2737.

Figures 30 through 37 identify potential EVSE locations for the balance of Commands monitored vehicles as noted in their captions.

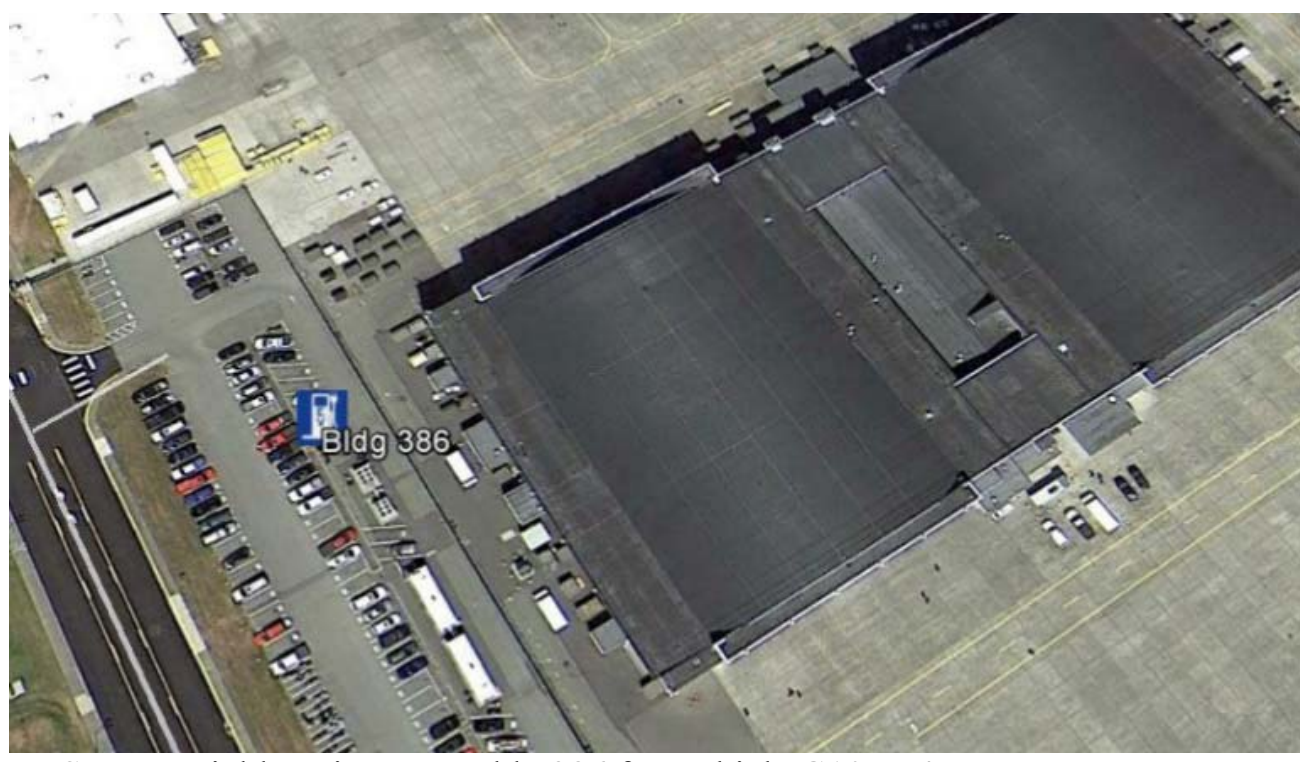

Figure 30. EVSE potential location near Bldg 386 for Vehicle G10-7547F. 


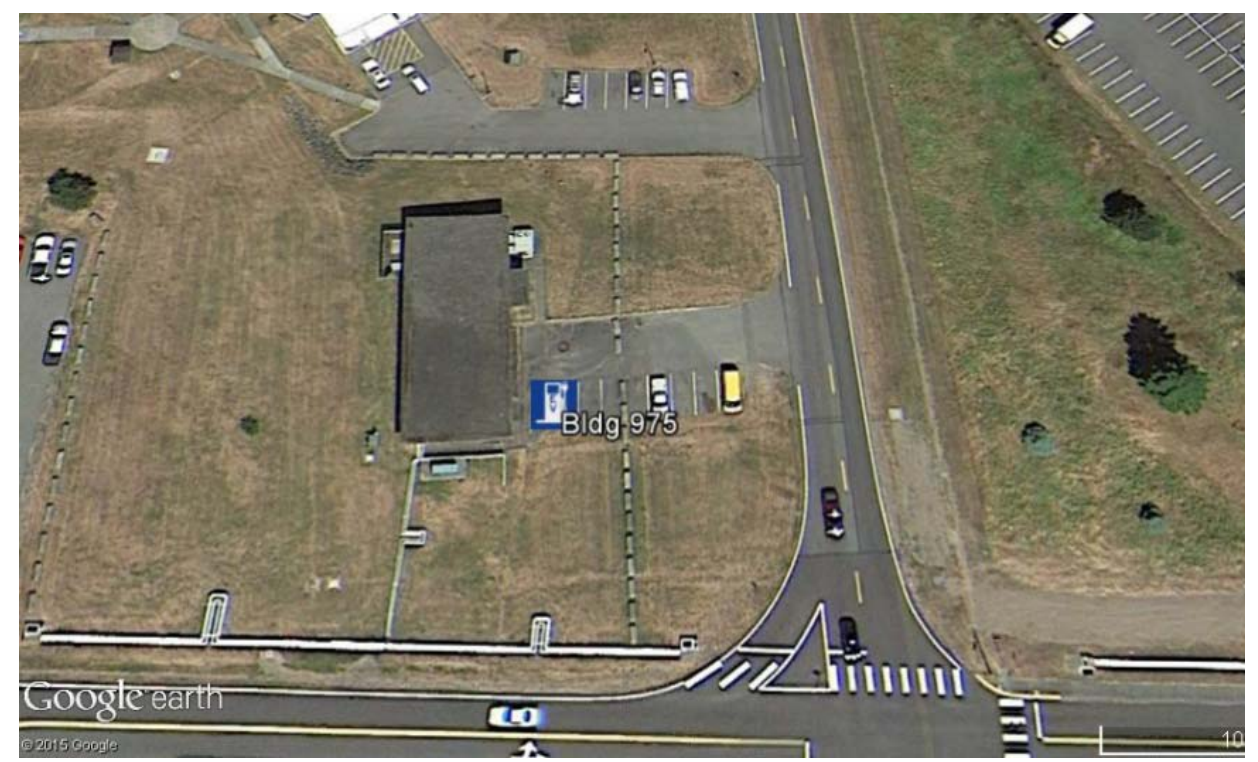

Figure 31. EVSE potential location near Bldg 975 for Vehicle G41-2911M.

Insufficient information was collected on Vehicle G41-3159P to specifically identify a home base. However, it was located near Building 124 the vehicle maintenance building. Several other Departments vehicles also park in this area.

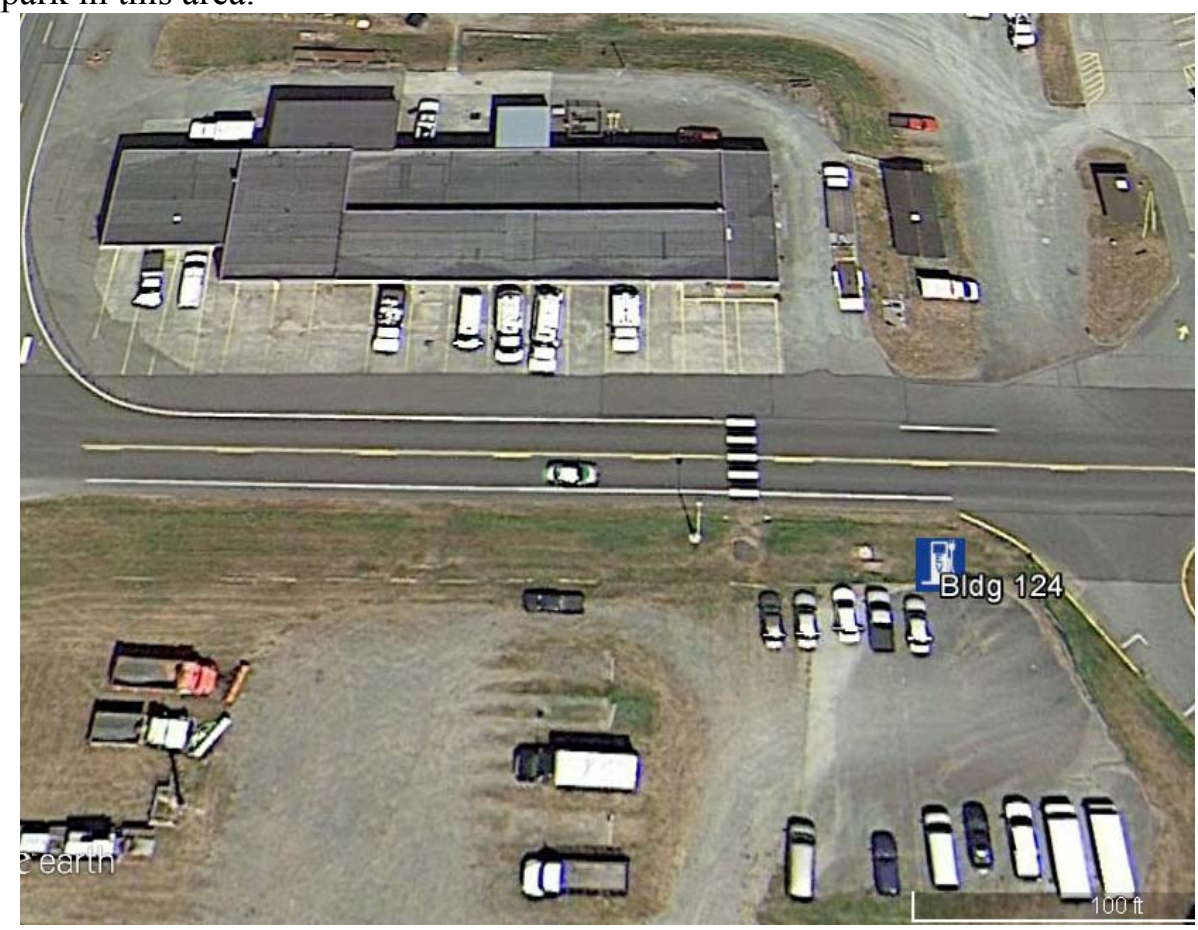

Figure 32. EVSE potential location near Bldg 124 for Vehicle G41-3159P. 


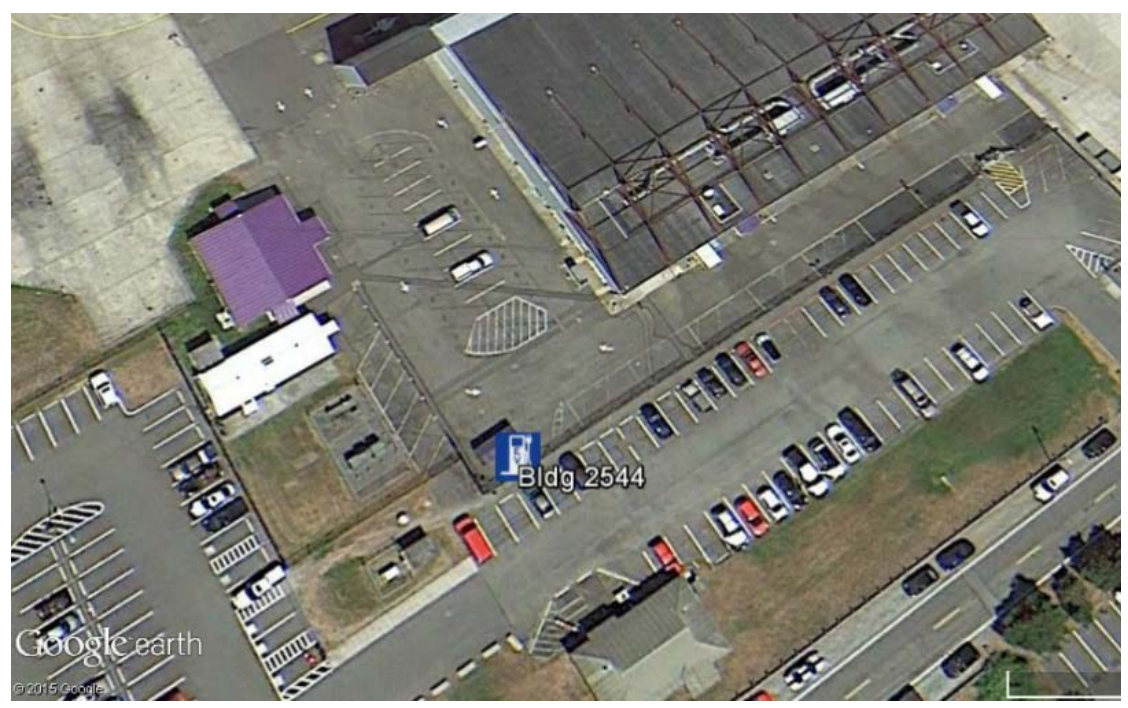

Figure 33. EVSE potential location near Bldg 2544 for Vehicle G41-1351G.

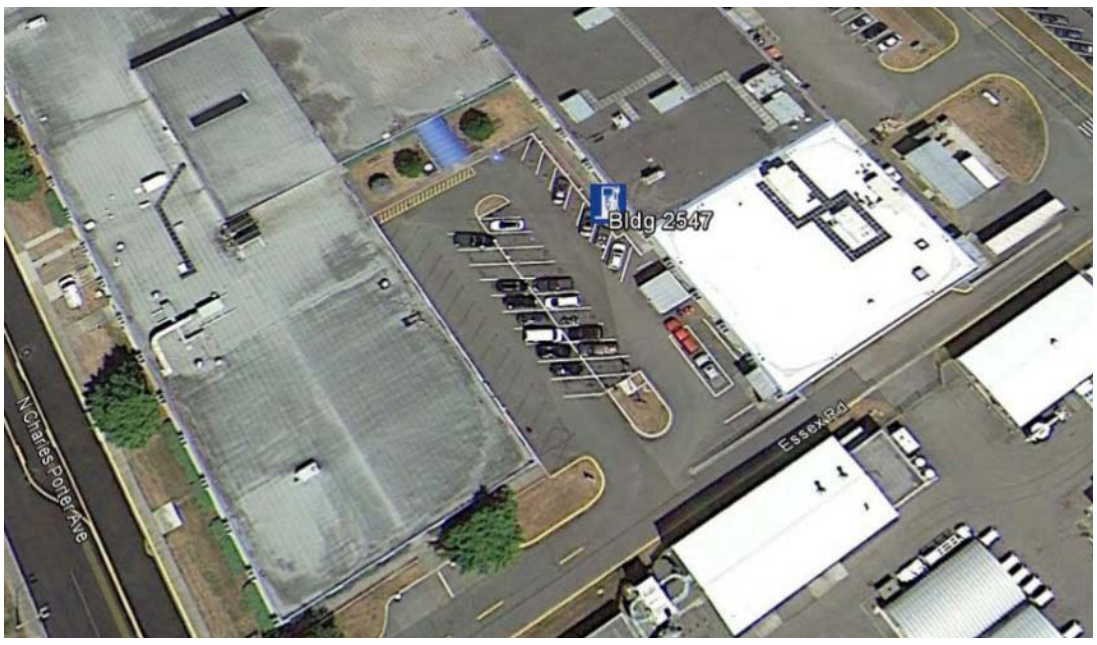

Figure 34. EVSE potential location near Bldg 2547 for Vehicle G41-1136K.

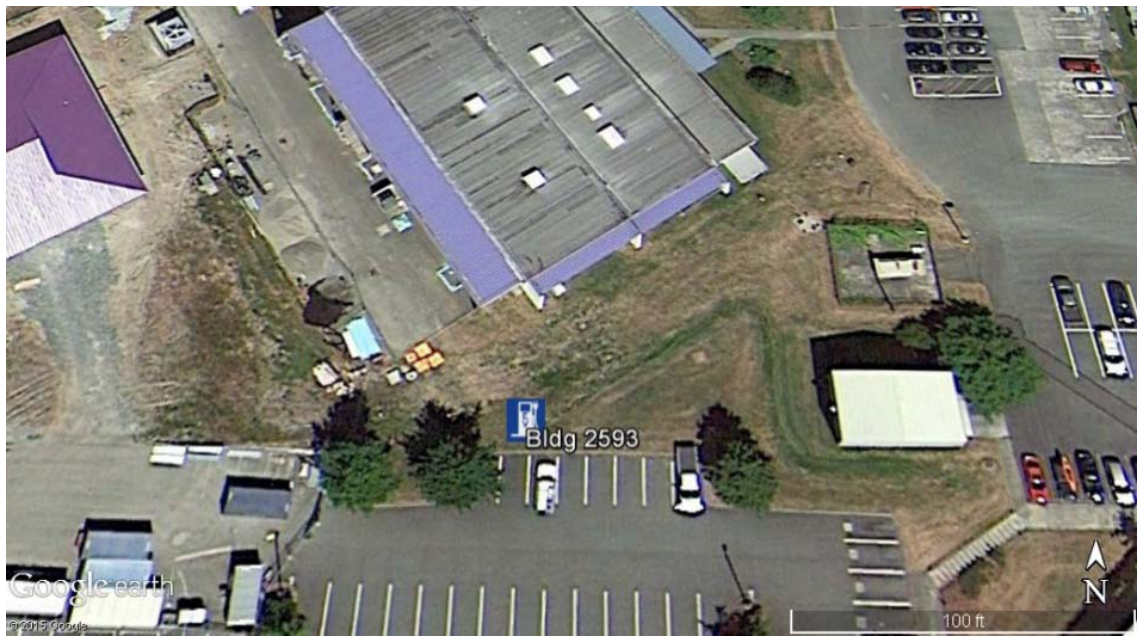

Figure 35. EVSE potential location near Bldg 2593 for Vehicle G10-1140M. 


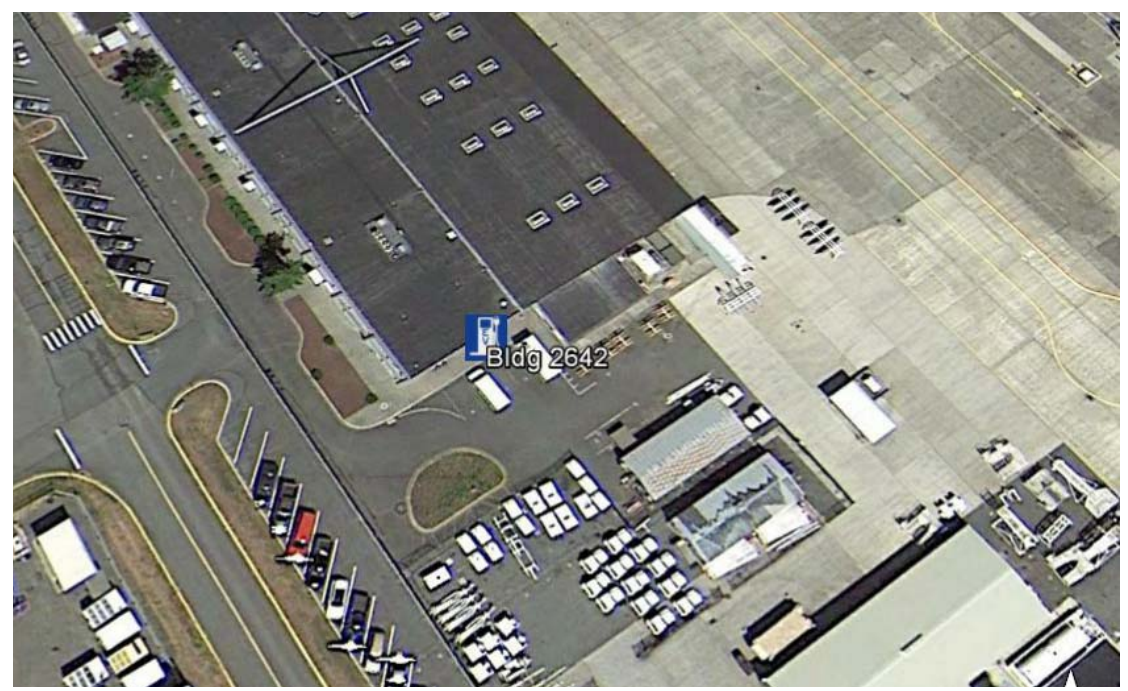

Figure 36. EVSE potential location near Bldg 2642 for Vehicle G43-3437B.

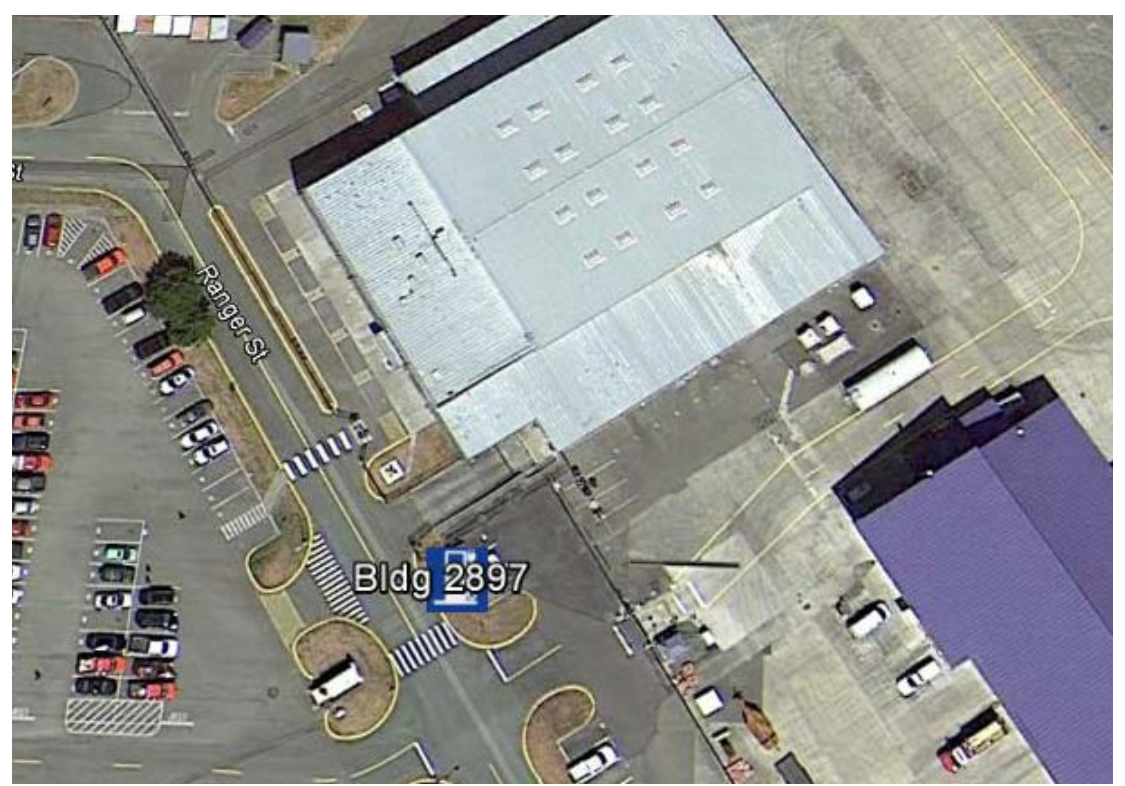

Figure 37. EVSE potential location near Bldg 2897 for Vehicle G61-0513K.

\subsubsection{Departments Group Home Base Electric Vehicle Supply Equipment Locations}

Table 11 identifies the home base locations for the Departments Group monitored vehicles.

Figure 38 identifies a potential location near Building 124. 


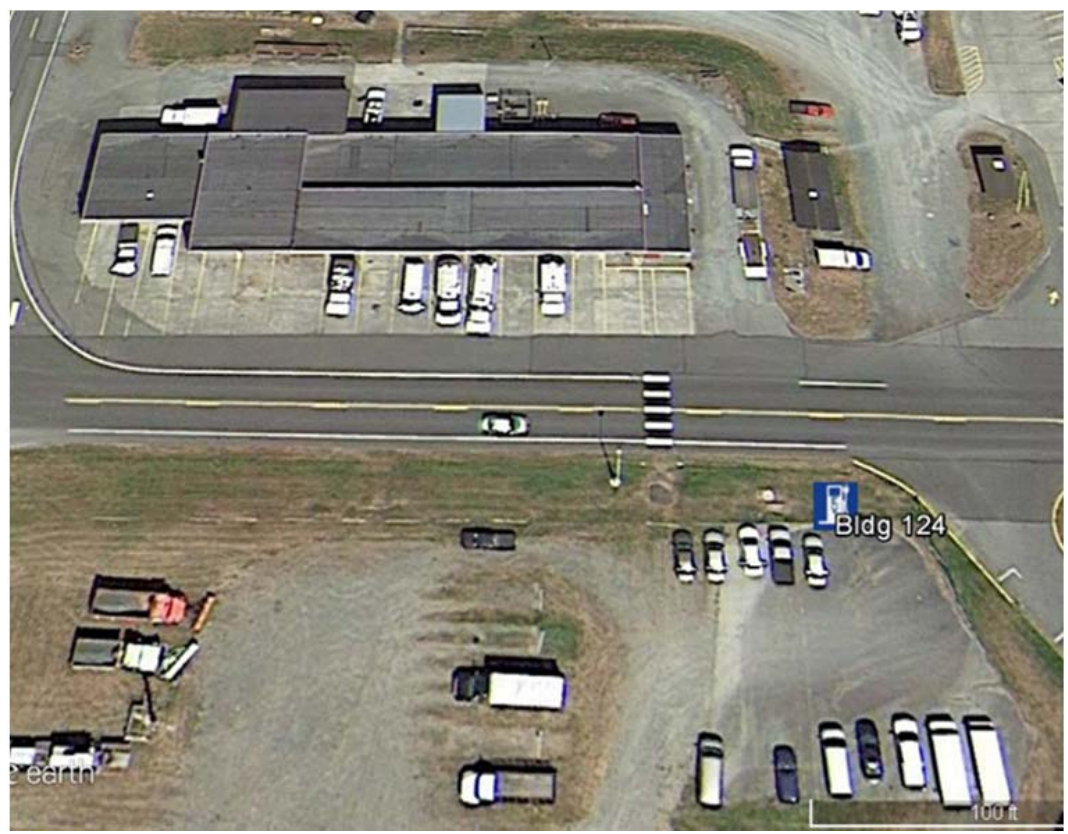

Figure 38. EVSE potential location near Bldg 124 for numerous vehicles.

Eight vehicles are home-based near this building: Pool Vehicles G10-2844L, G10-2848L, G102850L, G10-2968L, G42-1940N, G61-0245G, G42-0334G, and G42-0881H. The building is a vehicle maintenance building so installing the EVSE adjacent to the building may not be the most convenient because of frequent vehicle movements. Large parking areas are located as shown in Figure 38 as well as north of the building. Trenching power to any location will be required. The suggested location can be segregated for multiple EVSE for charging these vehicles.

Figure 39 identifies a potential location near Building 103. Several vehicles are home-based near this building: G41-1536L, G41-1597L, G41-1767H, G42-0656K, and G62-0203H. Most of these vehicles typically park in the parking lot to the south of the building. However, EVSE installed in this lot would require substantial asphalt cutting and trenching. EVSE parking to the east of the building may reduce installation costs. 


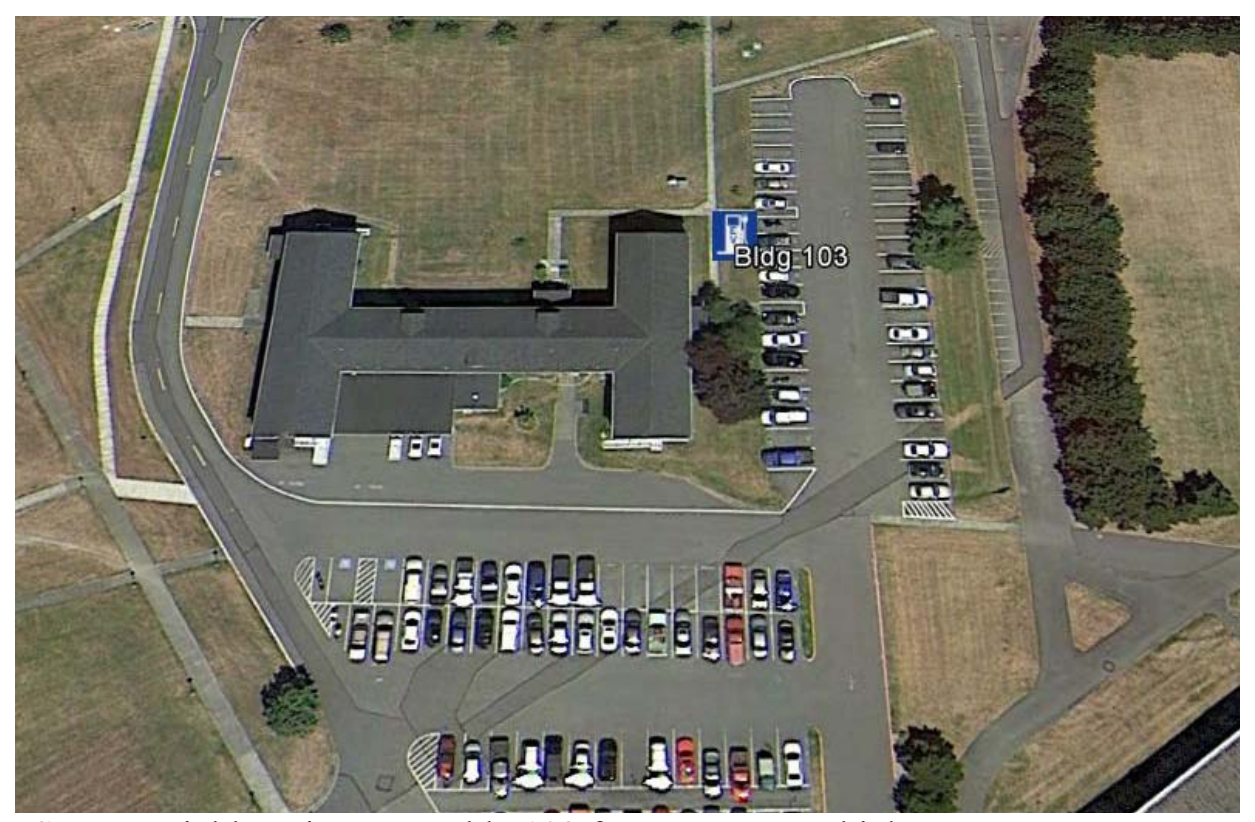

Figure 39. EVSE potential location near Bldg 103 for numerous vehicles.

Figure 40 identifies a potential location near Building 994.

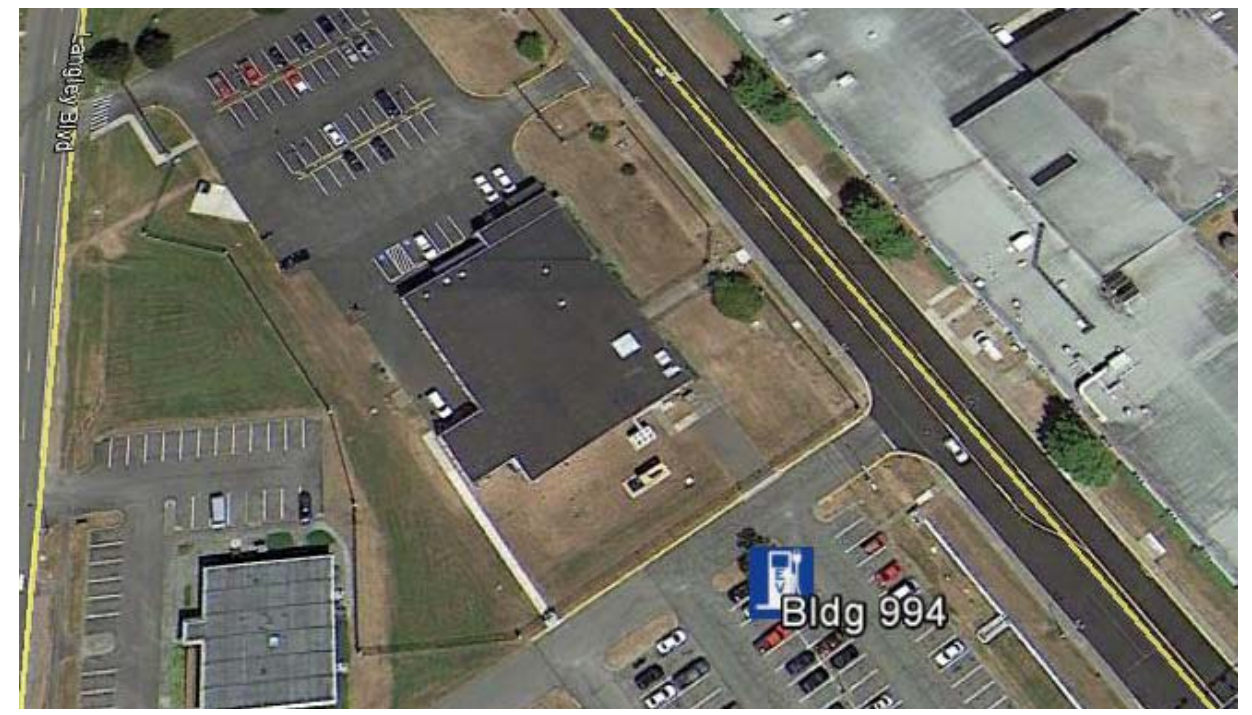

Figure 40. EVSE potential location near Bldg 994 for numerous vehicles.

Enforcement Vehicles G11-0472L, G11-1424L, G11-2898K, G42-2035L, G62-0871K, and G62$2420 \mathrm{P}$ assigned to Departments are home based near this building. Typically, these vehicles parked in the parking lot to the northwest of this building. However, placing EVSE in that main lot, unless directly adjacent to the building, would require extensive asphalt cutting and trenching. The number of parking spaces adjacent to the building would be insufficient for these vehicles or future additions. The parking lot to the southeast provides the greatest flexibility for future PEV growth.

Figure 41 identifies a potential location near Building 385. Vehicles G41-1142K, G41-1350G, G411765H, G41-1768H, G42-1222M, and G43-2288M are home based near this building. Several Commands vehicles also park near this building. 


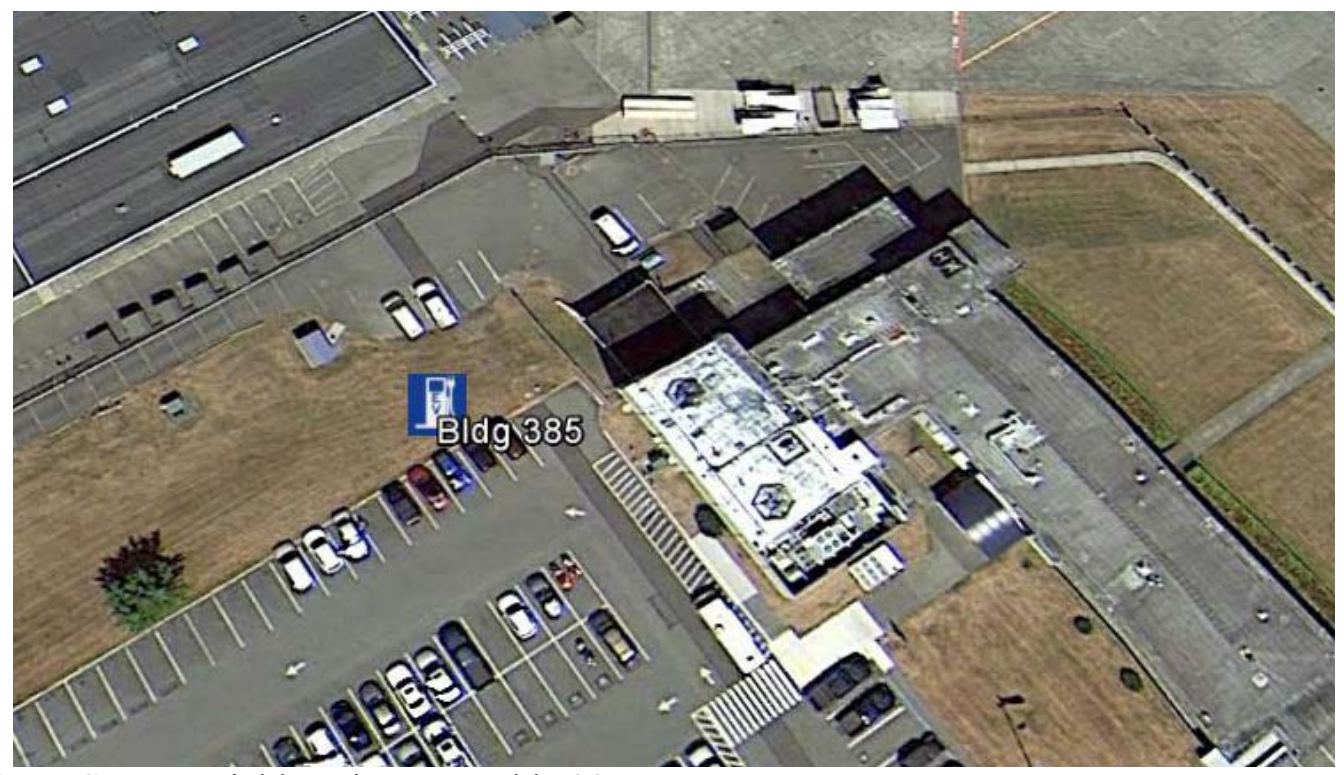

Figure 41. EVSE potential location near Bldg 385.

Figure 42 identifies a potential location near Building 2734. Vehicles G41-1763H, G42-1223M, G430895K, and G43-0896K are home based near this building.

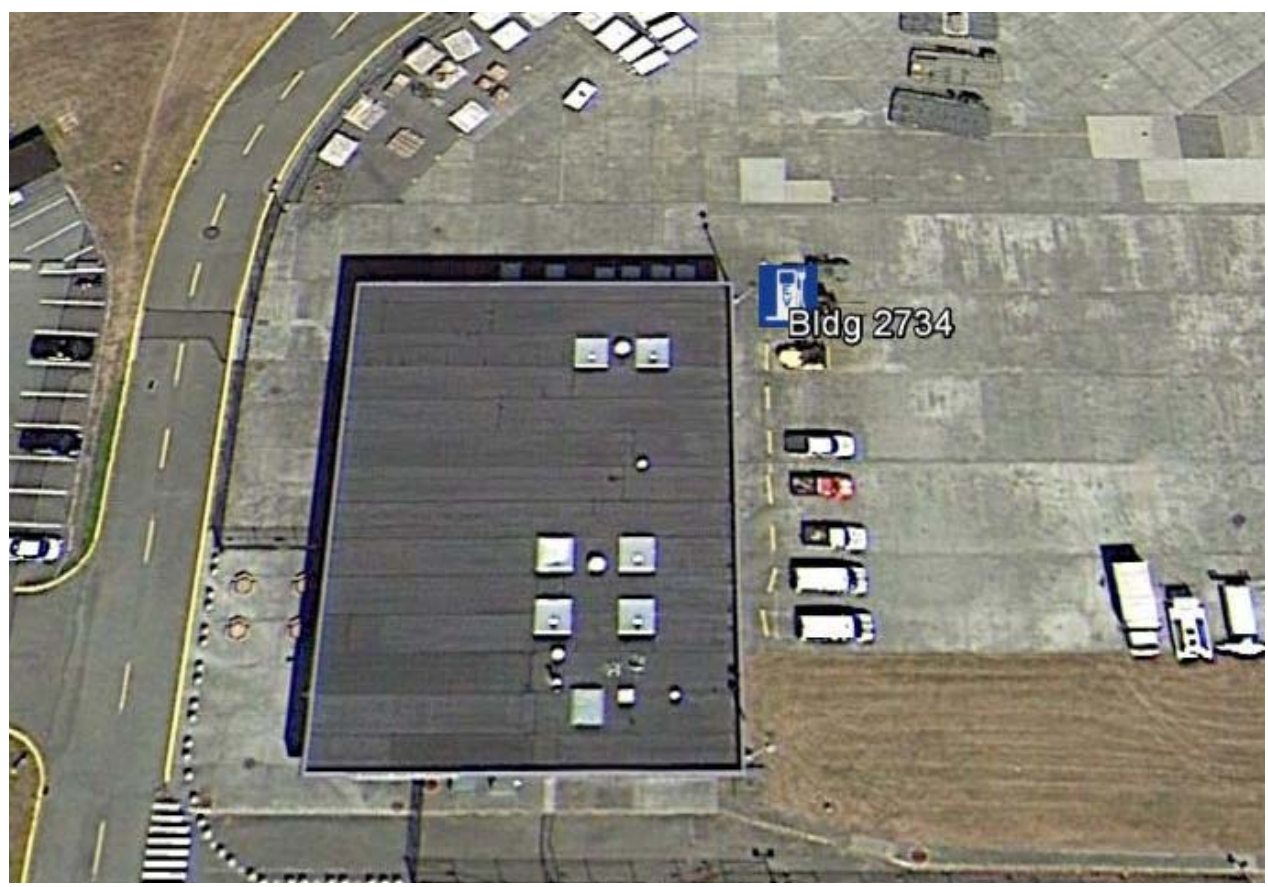

Figure 42. EVSE potential location near Bldg 2734 for various vehicles.

Figure 43 identifies a potential location near Building 243. Vehicles G10-1137M and G10-3590P are home based near this building. 


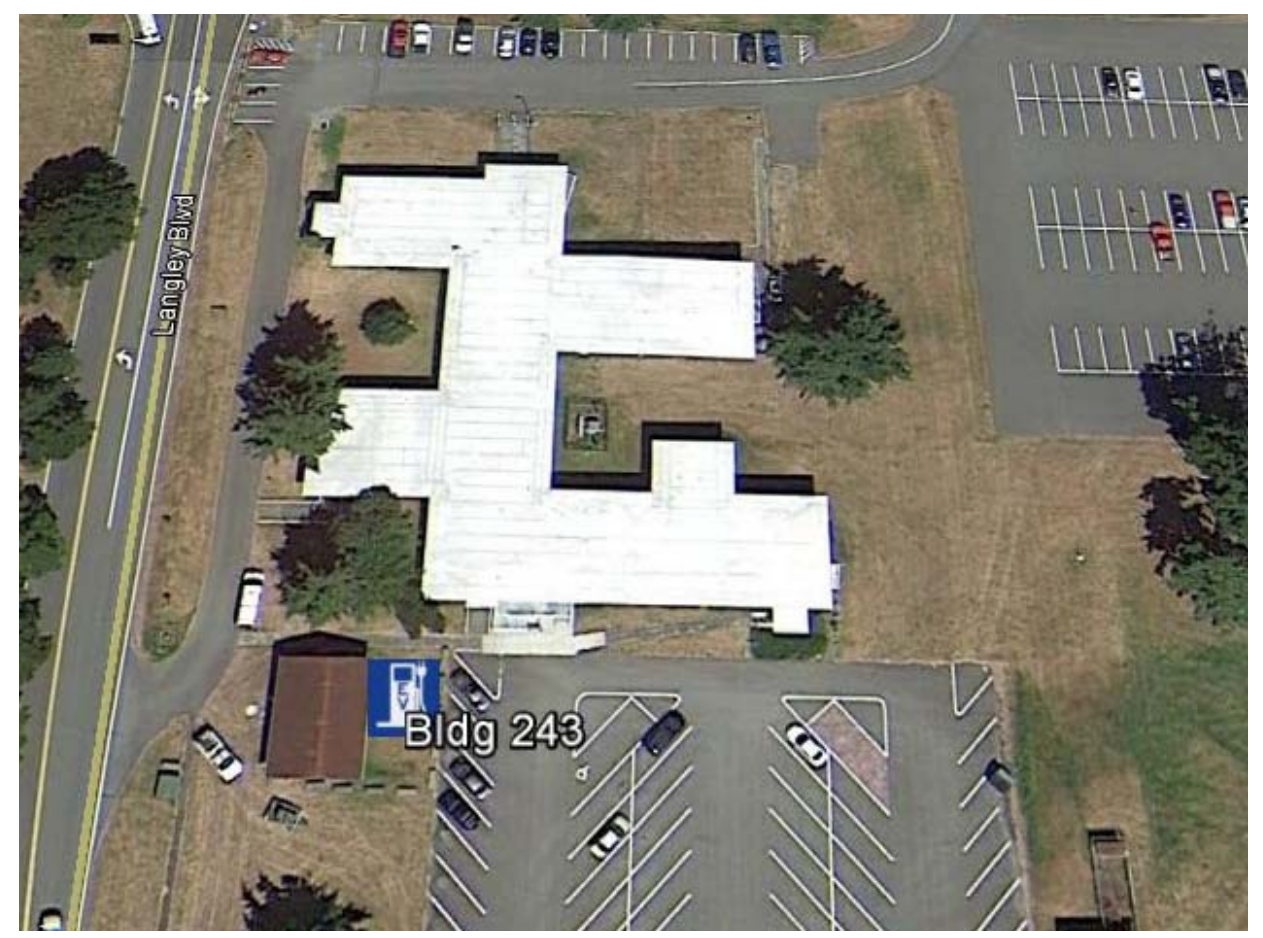

Figure 43. EVSE potential location near Bldg 243 for various vehicles.

Figures 44 through 52 identify potential EVSE locations for the balance of Departments monitored vehicles as noted in their captions.

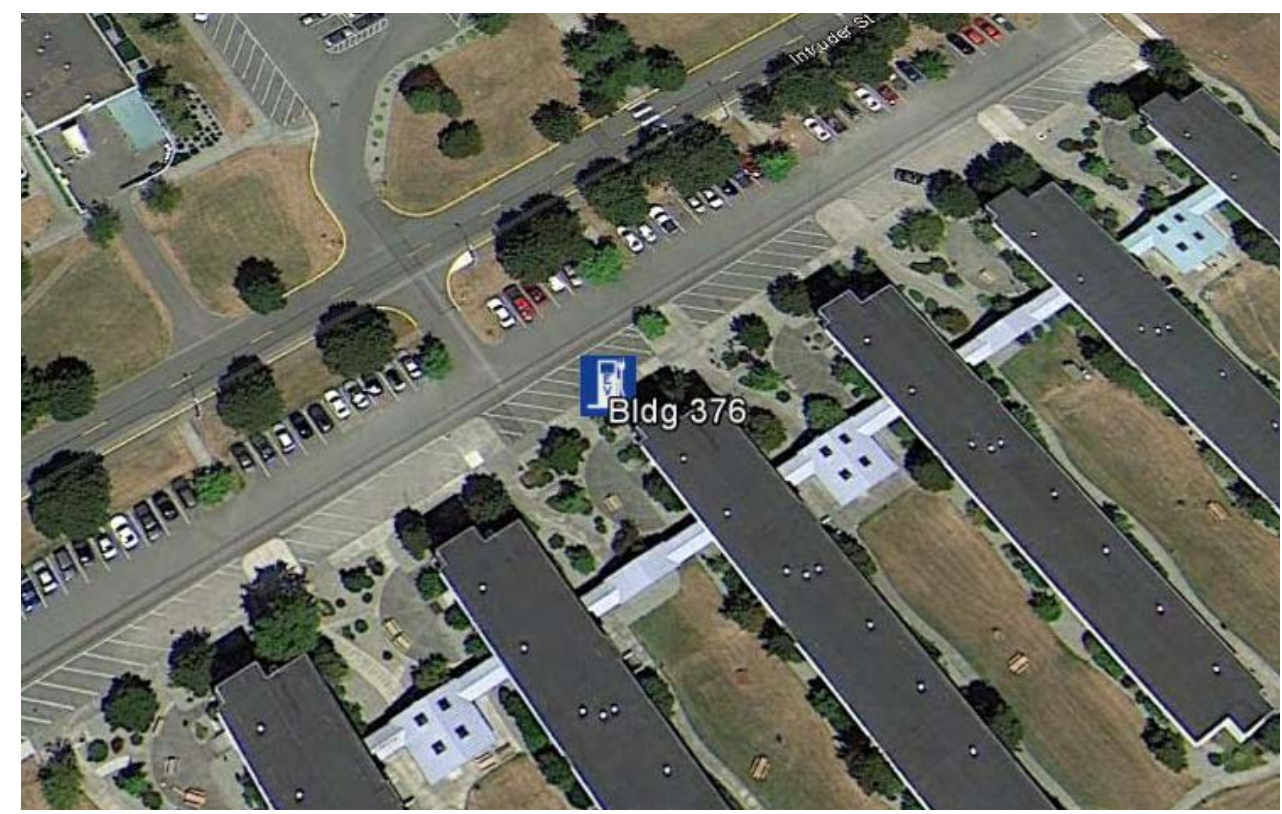

Figure 44. EVSE potential location near Bldg 376 for Vehicle G41-1349G.

Vehicle G10-0984N typically parks in the parking lot to the west of Bldg 382. However, installation of EVSE in that parking lot would require extensive asphalt cuts and long conduit runs. The lot to the south appears to be much closer to the electrical service with much shorter conduit runs. 


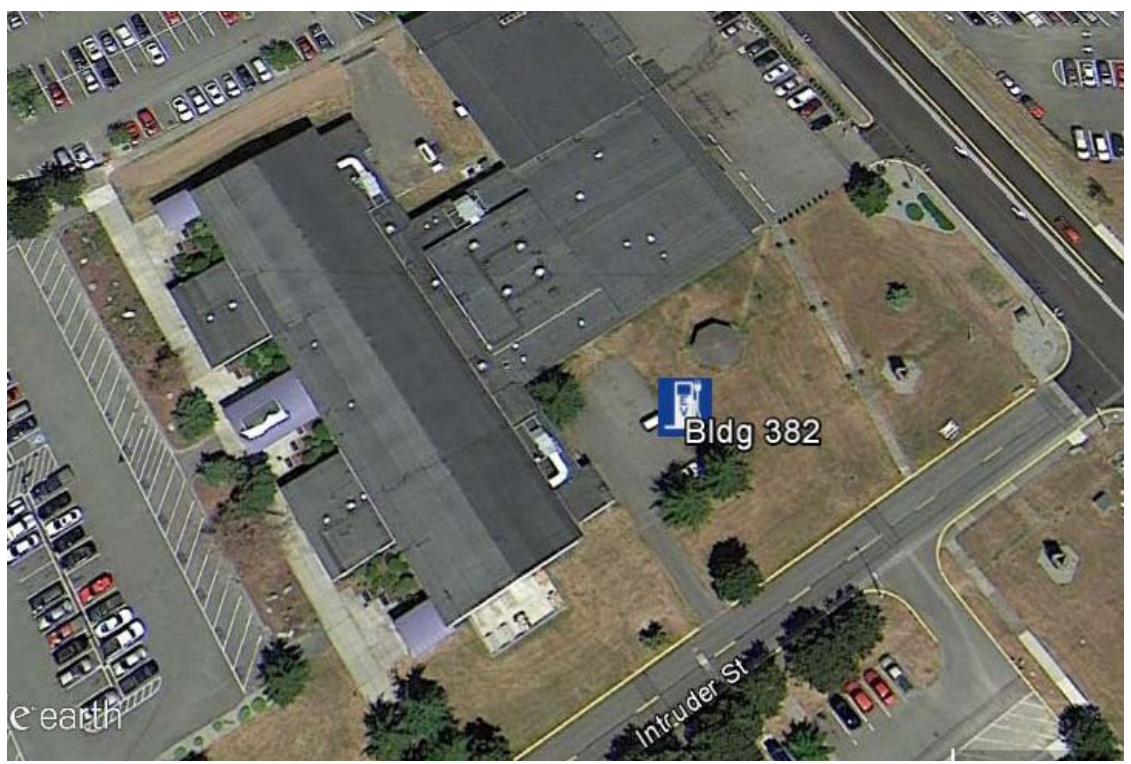

Figure 45. EVSE potential location near Bldg 382 for Vehicle G10-0984N.

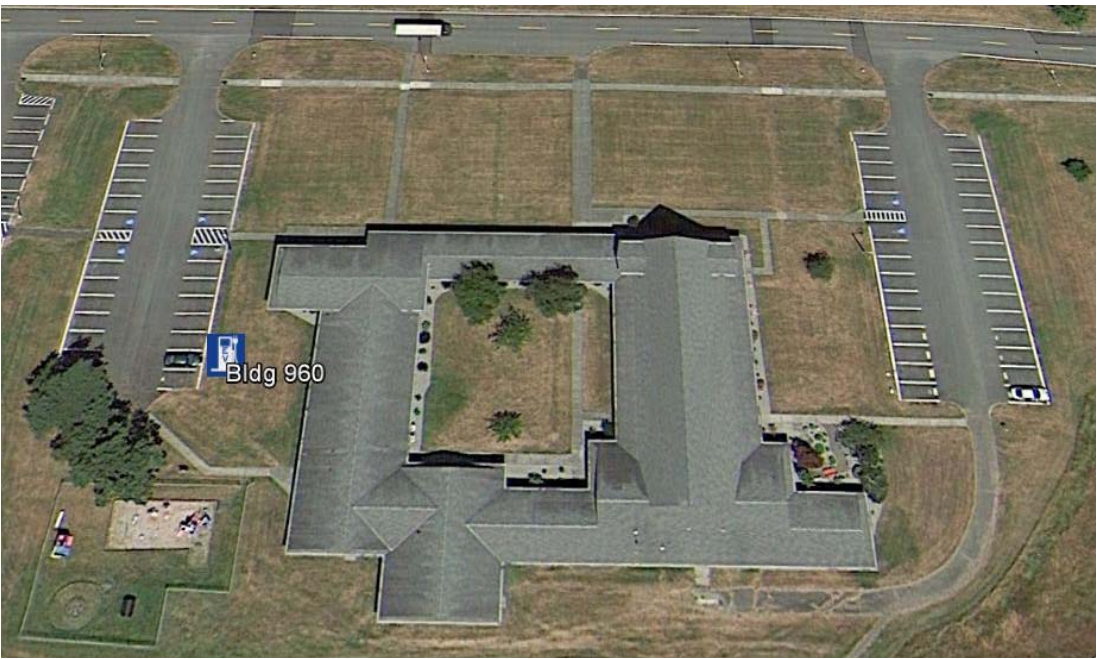

Figure 46. EVSE potential location near Bldg 960 for Vehicle G10-5286H.

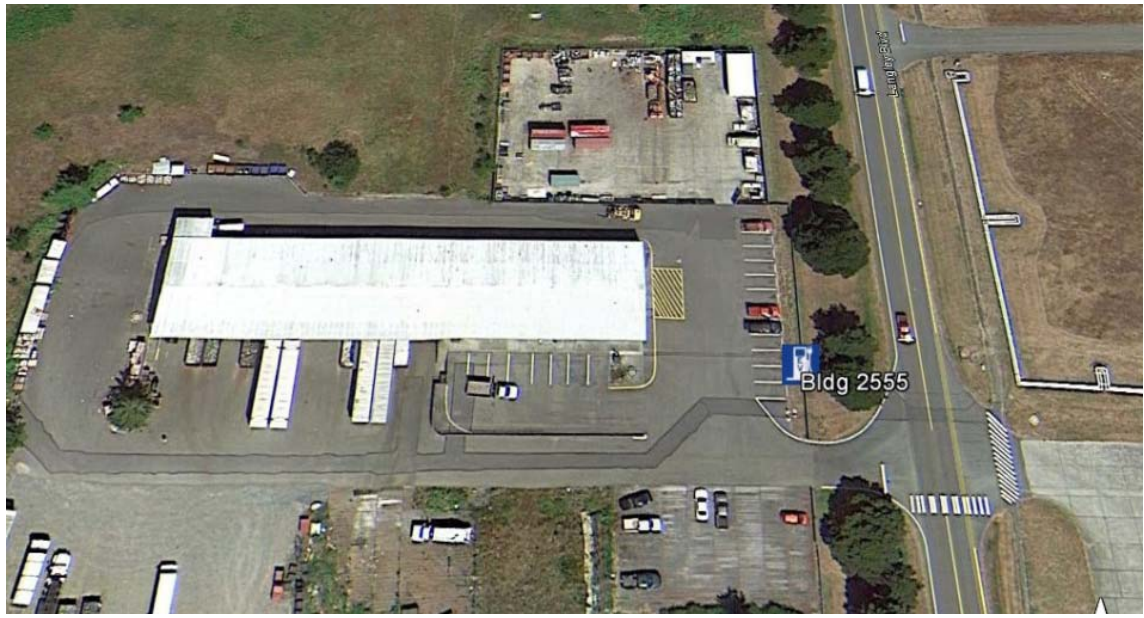

Figure 47. EVSE potential location near Bldg 2555 for Vehicle G42-0766L. 


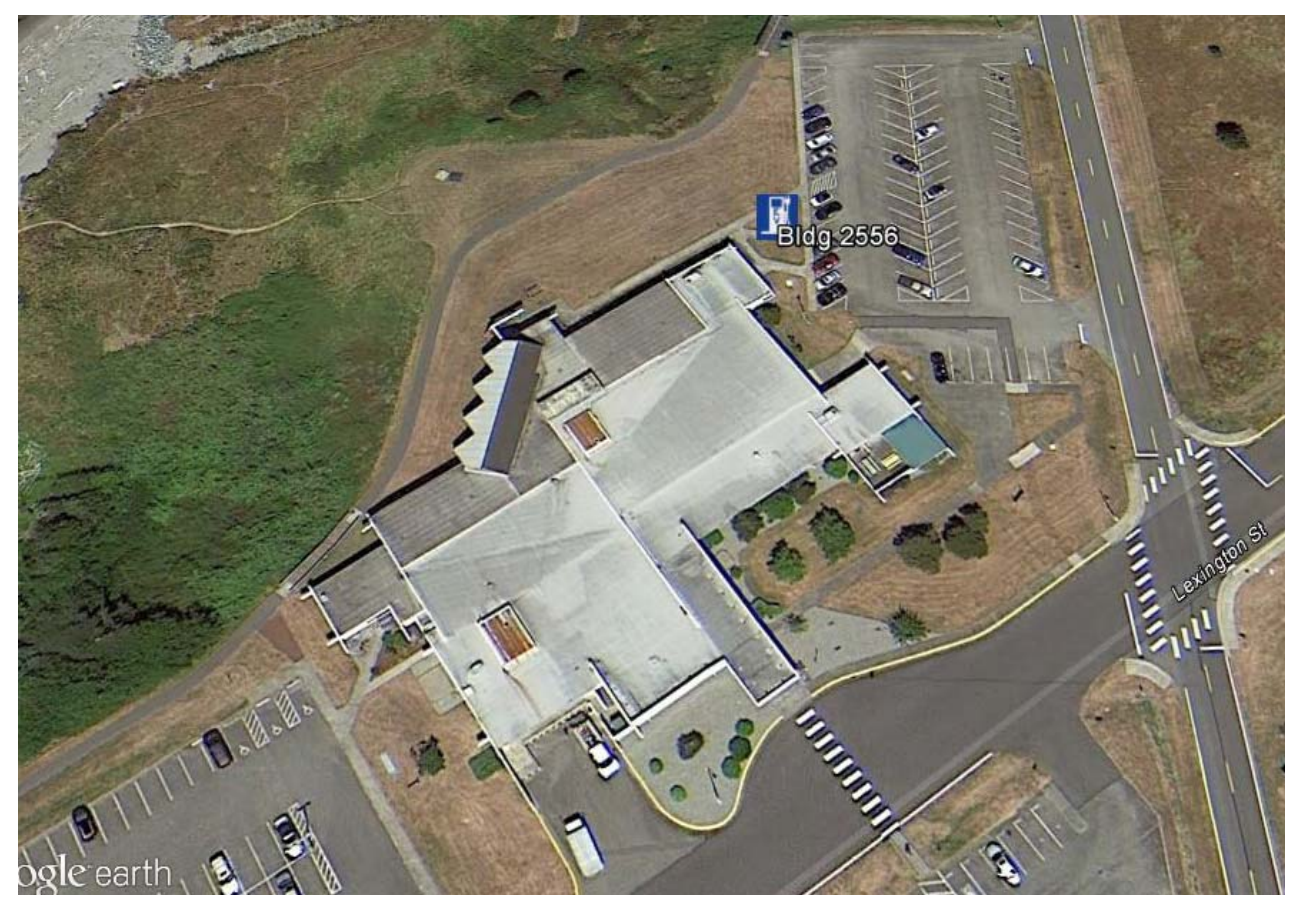

Figure 48. EVSE potential location near Bldg 2556 for Vehicle G41-1137K.

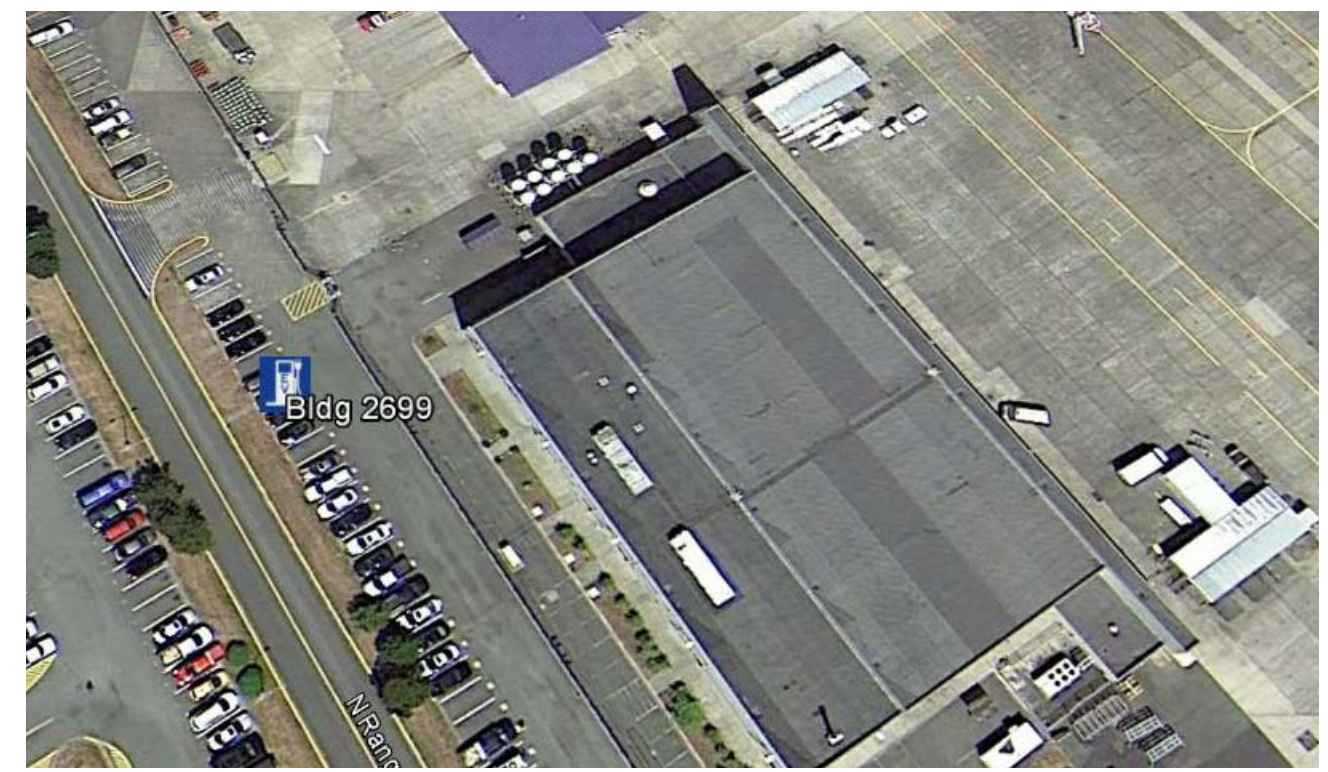

Figure 49. EVSE potential location near Bldg 2699 for Vehicle G41-4334M. 


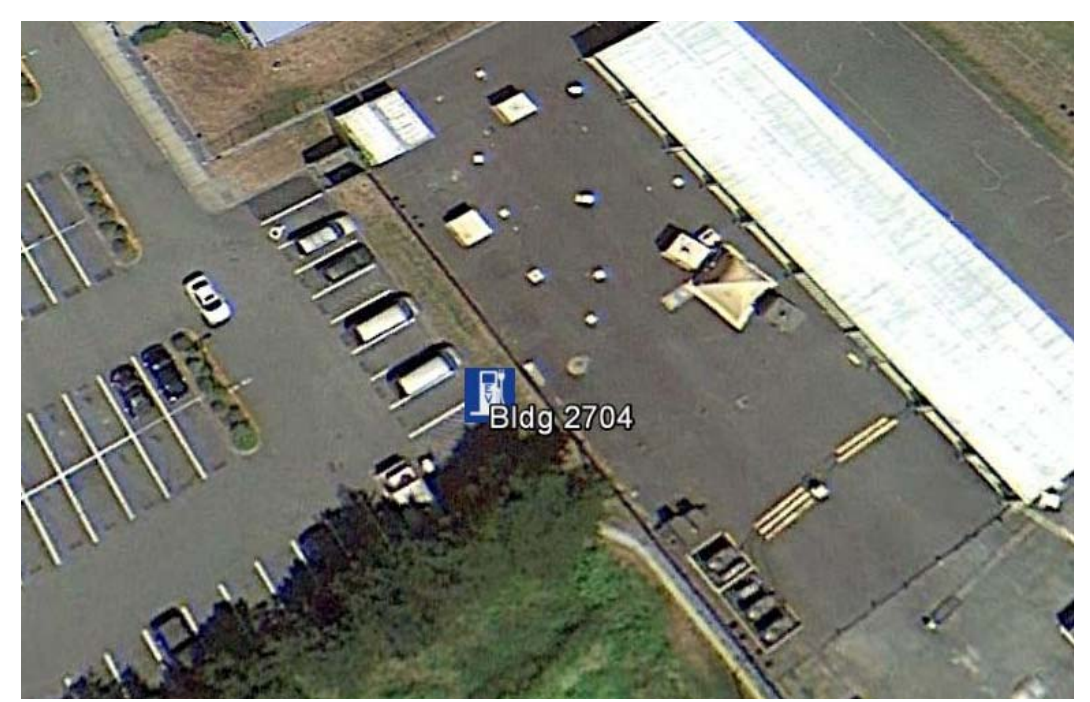

Figure 50. EVSE potential location near Bldg 2704 for Vehicle G42-0335G.

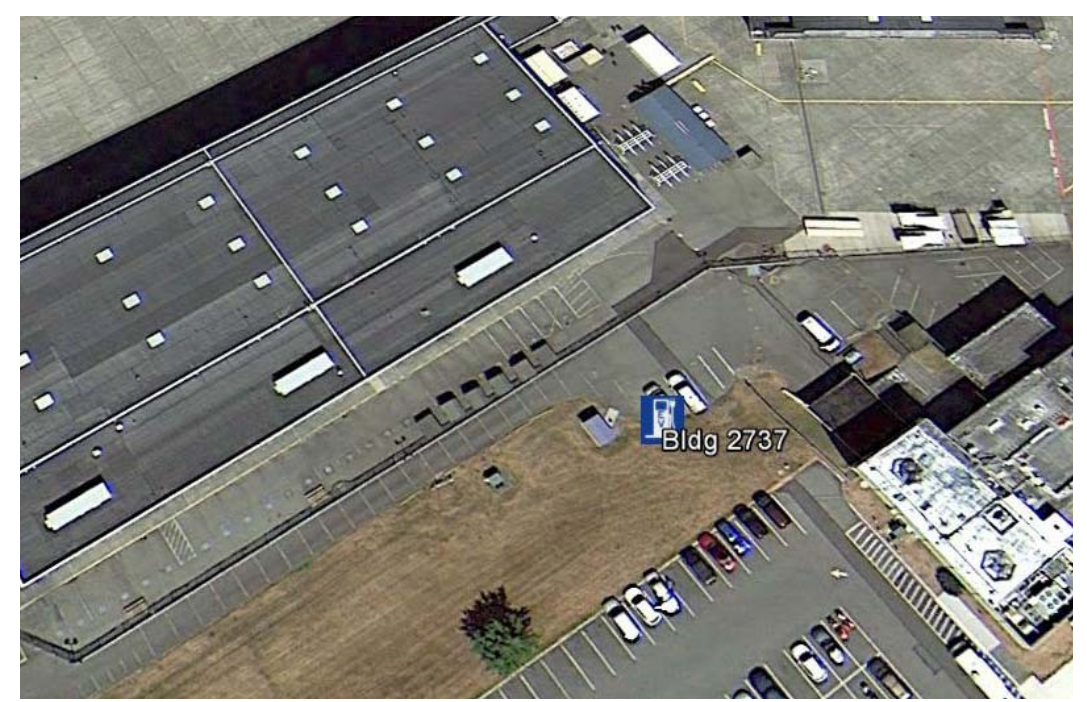

Figure 51. EVSE potential location near Bldg 2737 for Vehicle G43-2287M.

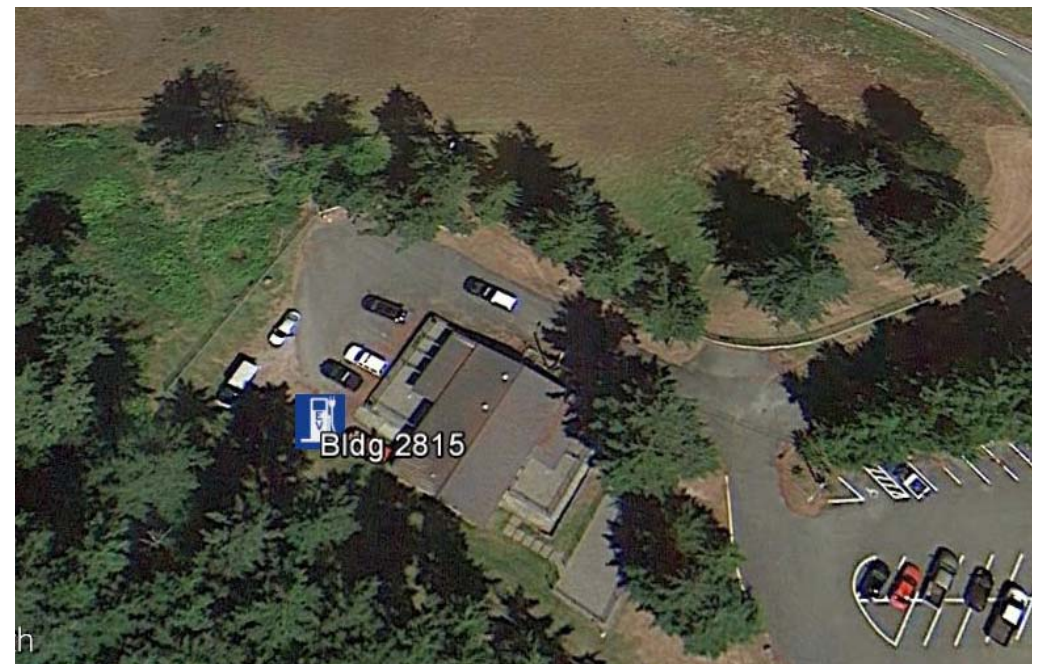

Figure 52. EVSE potential location near Bldg 2815 for Vehicle G43-0936K. 


\section{OBSERVATIONS}

Intertek appreciates the opportunity to present the results of this evaluation. Observations reported herein provide input to the next phases of this study. Specifically:

- The Task 3 Vehicle Utilization report and this report suggest PEV replacements and the identification of charging infrastructure needs and locations. This information will provide input to the Task 4 effort.

- Suggested PEV replacements can be considered with vehicle age to prepare a replacement schedule as part of Task 4 .

- The vehicle replacement schedule will dictate the charging infrastructure deployment schedule.

- Vehicle and EVSE schedules can factor into budget considerations for implementing vehicle replacements.

- Vehicle and EVSE schedules can factor into base objectives in fuel cost reductions and GHG emissions reductions.

The analysis shows the average vehicle travels approximately 5,090 miles per year. This is an average of less than 100 miles per week. This was considered here to reduce the number of AC Level 2 EVSE and thus reduce EVSE costs.

Intertek suggests NASWI may wish to move forward in the near future with the replacement of pool, support, and enforcement vehicles with PEVs as current budget and vehicle replacement schedules allow. Certainly, the vehicle types studied in this report may be candidates for immediate replacement. 